University of Tennessee Health Science Center UTHSC Digital Commons

\title{
Effect of Background Synaptic Activity on Excitatory-Postsynaptic Potential-Spike Coupling
}

\author{
Veronika Zsiros \\ University of Tennessee Health Science Center
}

Follow this and additional works at: https://dc.uthsc.edu/dissertations

Part of the Medical Neurobiology Commons, and the Neurosciences Commons

\section{Recommended Citation}

Zsiros, Veronika, "Effect of Background Synaptic Activity on Excitatory-Postsynaptic Potential-Spike Coupling" (2003). Theses and Dissertations (ETD). Paper 327. http://dx.doi.org/10.21007/ etd.cghs.2003.0387.

This Dissertation is brought to you for free and open access by the College of Graduate Health Sciences at UTHSC Digital Commons. It has been accepted for inclusion in Theses and Dissertations (ETD) by an authorized administrator of UTHSC Digital Commons. For more information, please contact jwelch30@uthsc.edu. 


\title{
Effect of Background Synaptic Activity on Excitatory-Postsynaptic Potential-Spike Coupling
}

\author{
Abstract \\ Neurons receive large amount of synaptic inputs in vivo, which may impact the coupling between EPSPs \\ and spikes. We mimicked the in vivo synaptic activity of the cell with the dynamic clamp system. We \\ recorded from pyramidal cells in neocortical slices in vitro to investigate how timing and probability of \\ spike generation in response to an EPSP is affected by background synaptic conductance under these \\ conditions. We found that near threshold, background synaptic conductance improved the precision of \\ spike timing by reducing the depolarization-related prolongation of the EPSP. In cells with ongoing spike \\ activity and background synaptic conductances, an EPSP rapidly increased the probability of firing. The \\ time window of the spike probability increase was comparable to the EPSP rise time and was followed by \\ a long period of reduced firing. We found that the net synaptic gain was determined not only by the \\ amplitude of the EPSP, but also by the firing frequency of the cell. In addition, a background fluctuating \\ conductance reduced the time window of perturbation of spike patterns generated by EPSP related \\ spikes. Taken together, these results indicate that in vivo, the level of the background synaptic activity \\ may regulate spike-timing precision and affect synaptic gain.

\section{Document Type \\ Dissertation} \\ Degree Name \\ Doctor of Philosophy (PhD) \\ Program \\ Anatomy and Neurobiology \\ Research Advisor \\ Shaul Hestrin, Ph.D. \\ Keywords \\ cortex, pyramidal cell, network activity, EPSP, spike, evoked EPSP, dynamic clamp, spike-timing precision, \\ synaptic gain, information coding \\ Subject Categories \\ Medical Neurobiology | Medical Sciences | Medicine and Health Sciences | Neurosciences
}




\title{
EFFECT OF BACKGROUND SYNAPTIC ACTIVITY ON EXCITATORY- POSTSYNAPTIC POTENTIAL-SPIKE COUPLING
}

\author{
A Dissertation \\ Presented for \\ The Graduate Studies Council \\ The University of Tennessee \\ Health Science Center
}

\author{
In Partial Fulfillment \\ Of the Requirements for the Degree \\ Doctor of Philosophy \\ From The University of Tennessee
}

By

Veronika Zsiros

December 2003 


\section{DEDICATION}

To my mother 


\section{ACKNOWLEDGEMENTS}

Many mentors and colleagues contributed to my work summarized in this dissertation during the years of my studies. I would like to thank particularly to my advisor professor, Dr. Shaul Hestrin to give me the opportunity to work on this project, providing me an environment

for developing my scientific skills and for his guidance and encouragement when things seemed to fall apart.

I am also grateful to my colleagues: Dr. Mario Galarreta and Dr. Zhigou Chu for their critical comments, and helpful discussion of the results and problems I encountered in the lab, not forgetting our wonderful lab assistant, Jane Li.

I also would like to gratefully acknowledge the contributions of the student advisory committee Dr. William Armstrong, Dr. Randall Nelson, Dr. Callaway, Dr. Robert Foehring and Dr. Tavalin. 


\begin{abstract}
Neurons receive large amount of synaptic inputs $n$ vivo, which may impact the coupling between EPSPs and spikes. We mimicked the in vivo synaptic activity of the cell with the dynamic clamp system. We recorded from pyramidal cells in neocortical slices in vitro to investigate how timing and probability of spike generation in response to an EPSP is affected by background synaptic conductance under these conditions.

We found that near threshold, background synaptic conductance improved the precision of spike timing by reducing the depolarization-related prolongation of the EPSP. In cells with ongoing spike activity and background synaptic conductances, an EPSP rapidly increased the probability of firing. The time window of the spike probability increase was comparable to the EPSP rise time and was followed by a long period of reduced firing. We found that the net synaptic gain was determined not only by the amplitude of the EPSP, but also by the firing frequency of the cell. In addition, a background fluctuating conductance reduced the time window of perturbation of spike patterns generated by EPSP related spikes.

Taken together, these results indicate that in vivo, the level of the background synaptic activity may regulate spike-timing precision and affect synaptic gain.
\end{abstract}




\section{TABLE OF CONTENTS}

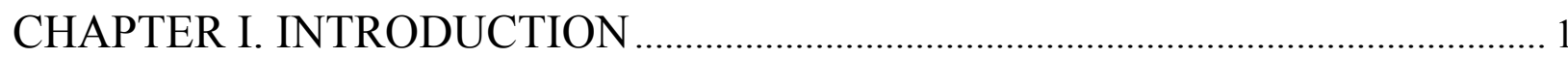

Strategies for decoding neuronal spike trains ............................................................. 1

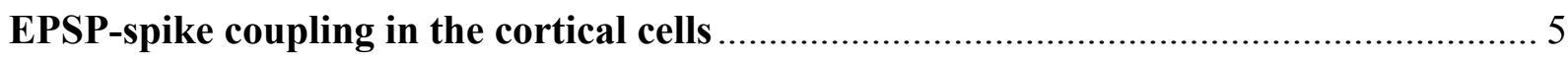

Effect of network activity on EPSP-spike coupling ……………….............................. 6

Response variability and ongoing synaptic activity in cortical cells ................................. 8

Functional consequences of ongoing synaptic activity ………........................................ 10

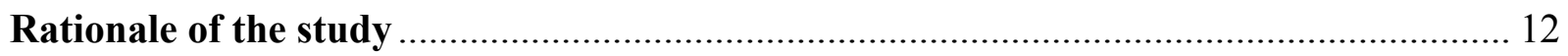

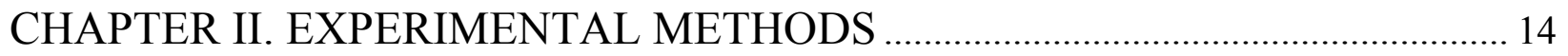

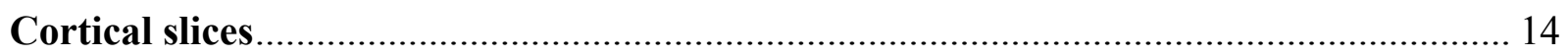

Selection criteria for recording from layer 5 pyramidal cells......................................... 15

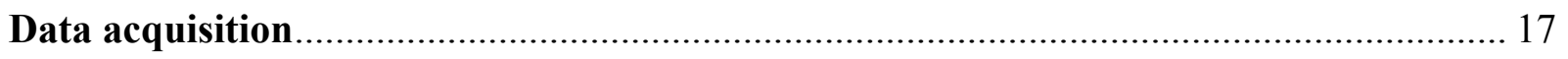

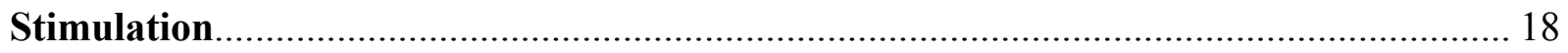

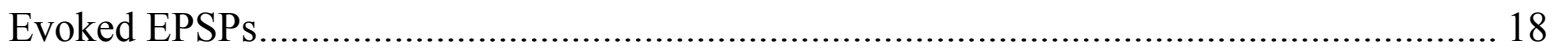

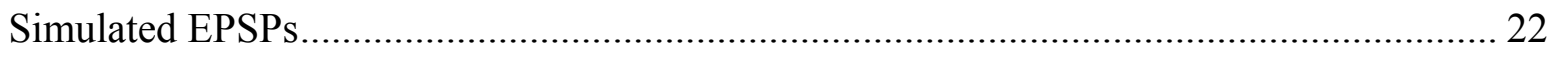

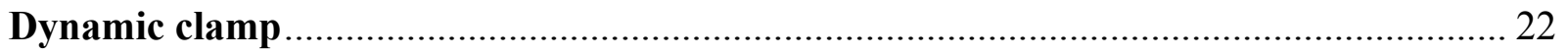

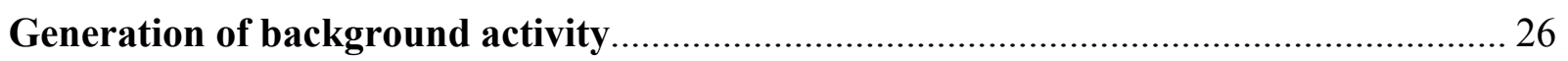

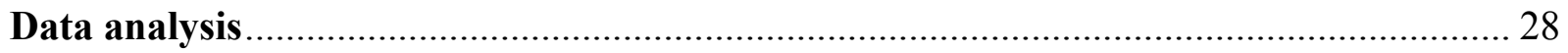

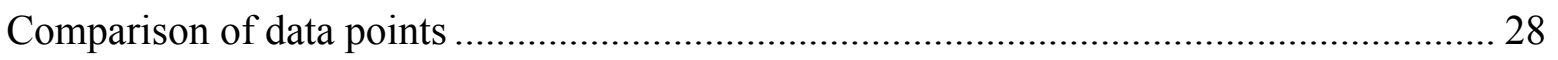

Calculation of peristimulus time histograms and cumulative sums................................... 29

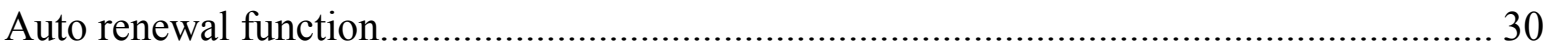

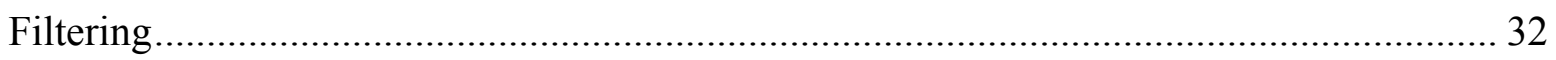


Anatomy

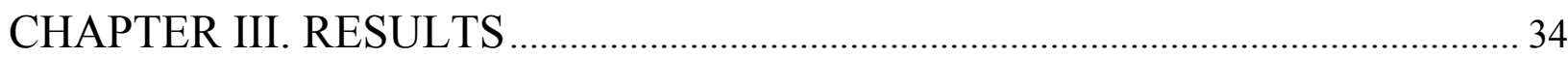

The effect of conductance on the properties of the cell ............................................ 34

Conductance reduces depolarization induced prolongation of EPSP........................... 37

Effect of membrane potential fluctuation and the amount of conductance on EPSP

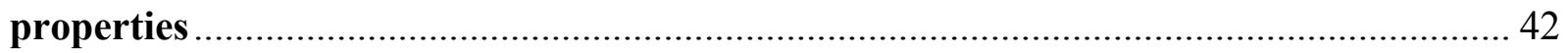

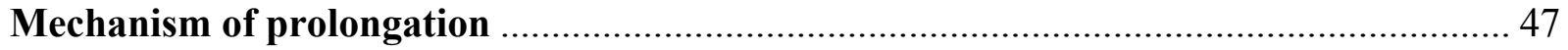

Background conductance improve spike timing precision ...................................... 49

Relationship between PSTH and EPSP properties............................................... 53

The role of fluctuation in the background activity ................................................ 62

Effect of fluctuating background conductance on perturbation of spike timing............ 65

CHAPTER IV. DISCUSSION ....................................................................... 73

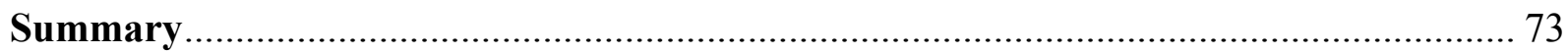

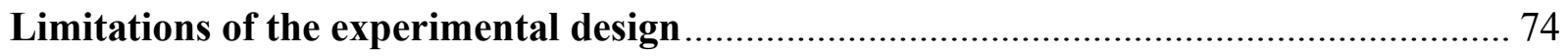

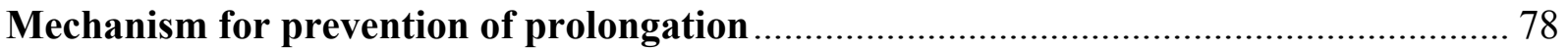

How much conductance is sufficient to improve the precision of spike timing? ............ 81

Refractory period and the effect of a single spike on the spike timing ........................ 83

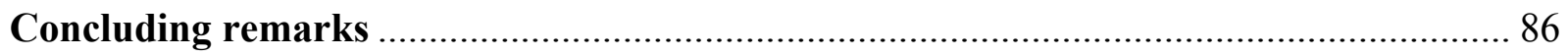

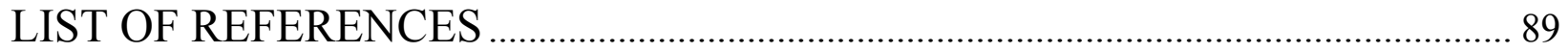

DEFINITIONS AND ABBREVIATIONS ............................................................ 104

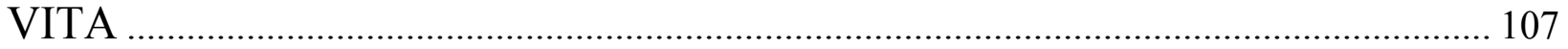




\section{LIST OF FIGURES}

Figure 1. Evoked EPSP in the cortex. 16

Figure 2. Comparison of spontaneous, simulated and evoked EPSPs on layer 5 pyramidal cells. 21

Figure 3. The data acquisition system used for dynamic clamp experiments. 23

Figure 4. Test of the dynamic clamp. 25

Figure 5. Generation of fluctuating background activity..... 27

Figure 6. The renewal density function. 31

Figure 7. Effect of injection of conductance on the membrane properties of the cell. 35

Figure 8. Effect of background synaptic conductance on the properties of eEPSP. 38

Figure 9. Effect of background synaptic conductance on the properties of simulated EPSP.

Figure 10. Effect of tonic and fluctuating conductance on EPSP half width. 44

Figure 11. Prolongation of simEPSP is related to the activation of sodium channels. 48

Figure 12. Effect of background conductance on the precision of spike timing. 51

Figure 13. Effect of eEPSP amplitude on peak synaptic gain........................................ 55

Figure 14. Relationship between firing frequency and spike loss. ............................... 60

Figure 15. Effect of fluctuating conductance on spike loss. . .......................................... 63

Figure 16. Perturbation of spike timing following an action potential generated by an EPSP. 


\section{CHAPTER I. INTRODUCTION}

\section{Strategies for decoding neuronal spike trains}

During the process of synaptic integration, synaptic inputs are combined and generate action potential patterns to transmit information. Activity from presynaptic fibers converges onto the dendrite and the soma of the neuron generating postsynaptic potentials. However, the membrane potential reaches the action potential threshold of the postsynaptic neuron only if the inputs temporally overlap during the integration window. In computational theories, neurons have two possible ways to decode the information they receive and create a message. Depending on the length of the integration window compared to the average interspike intervals, neurons can operate as a rate coding devices (long integration window) or detectors of coincidentally arriving synaptic inputs during the short integration window. There is extensive literature on coding in different neuronal systems from different perspectives (see references in deCharms and Zador (2000)).

In reality, transmission of information takes place in a noisy channel. This means there is always some error superimposed on the top of the "true signal" of the coded message. This error is referred as noise. Noise can be systematic or random. Examples for random noise are the opening of voltage gated ion channels that is probabilistic in nature and may limit the reliability of neuronal responses (White et al., 2000, White et al., 1998), or the low reliability of synaptic transmission (Hessler et al., 1993). Systematic noise could originate from processes that affect the nervous system in a more general way, like metabolic changes, global anesthesia, sleep and awake states or pathological conditions. Theoretically, the organization of the connectivity also may contribute to systematic noise in coding. Cortical cells have high degree of convergence and 
divergence in their synaptic connections. When numerous neurons are activated, a systematic noise can be generated from the interactions between responses unrelated to the stimuli.

In the case of rate coding, a multiple synaptic inputs contribute to a single spike, because the integration window is long. Hence, variability in the firing rate of the presynaptic elements due to a random noise is filtered out by the averaging process (Shadlen and Newsome, 1994). For this reason, rate coding performs robustly in the presence of random noise, but the strength of the system is its limitation too. Rate coding neurons have a restricted range of operation in terms of frequency, and their input-output transformation is relatively slow, which in turn may limit the capacity for transmitting information. Systematic noise in the communication also could be accumulated by temporal integration (Konig et al., 1996).

The view that neurons utilize rate coding gained support very early on with the introduction of recording of electric activity from nerve fibers. For example in his classical work on frog sciatic nerve, Adrian (1926) found that the frequency of nerve impulses increases as the weight (stimulus intensity) used for stimulation of the muscle spindle receptors increases. He also made it clear that the content of information transmitted depends on the nature of the axon stimulated and not on the nature of stimulus, because the specificity of stimuli evoked similar "action currents" only in nerve fibers connected to specific receptors of the given stimulus, but not in other axons that were not connected receptor.

Similar findings were reported for the cochlea, where the increase of the intensity of an acoustic stimulus of constant frequency increased the probability of firing within the dynamic range of the recorded unit (Rose et al., 1967). Firing rates are locked to the frequency and phase of the stimulus. 
Barlow (1972) provided a more elaborate concept for rate coding of sensory input, in which the frequency of firing in the sensory neuron represents the magnitude of the signal-tonoise ratio. The neuronal element is not noisy itself, but the external noise originates in the environment. Consequently, the only efficient way of coding can be achieved is when a single neuron integrates input signals over a longer interval, and visual features are represented by a minimum number of neurons. Examples of external noise in the visual system are photon absorption, a stochastic process, or the "crosstalk" between neurons as a result of their concurrent activity.

Evidence in support of the rate coding model includes the length of the membrane time constant, which may be too long to allow detection of coincident signals (Shadlen and Newsome, 1994). Also, spike trains with similar statistical properties to those recorded in vivo could be generated by a simple integrate and fire neuron with balanced inhibition and excitation. Thus, the timing of spikes does not have to be meaningful for efficient coding (Shadlen and Newsome, 1998).

The concept of the neuron as coincidence detector originated from von der Malsburg (1981) and Abeles (1982a and 1982b) to provide a possible model for the "binding problem". Binding refers to grouping sensory features together into a coherent representation of an object and separating it from other objects in the field of perception. If neurons are coincidence detectors, then the fine structure of the postsynaptic spike pattern represents the temporal pattern of its presynaptic inputs (Singer, 1993). In coincidence detectors, the only inputs that participate in spike generation are those that arrive during the short (ms) length integration window. The number of individual synaptic inputs necessary to generate a spike is lower compared to neurons with rate coding; because they are synchronized they produce an EPSP with a shorter rise-time, 
which promotes a temporally precise input-output transformation (Engel et al., 1992). The capacity to convey information is higher than with rate coding. However neurons become more susceptible to random error messages (Konig et al., 1996). For that reason, nonlinearities that allow the neuron to weigh the synaptic inputs and select the relevant ones could be important in the signal processing. Examples of possible mechanisms producing nonlinear summation of synaptic inputs are: (1) ion channels or synaptic receptors that can amplify the individual synaptic input (Stafstrom et al., 1984, Stuart and Sakmann, 1995, Thomson, 1997, Fricker and Miles, 2000, Stuart, 1999) or modify its time course (Magee, 1998, Magee, 1999, Hoffman et al., 1997, Williams and Stuart, 2000); (2) plastic modulation of the synaptic input depending on the activity of the neuron, e.g. during long term potentiation or depression (Bliss and Lomo, 1973, Hausser et al., 2001, Markram et al., 1997, Tsodyks and Markram, 1997, Abbott et al., 1997); and (3) local computing in the dendritic branches that in other ways, not mentioned under (1) and (2), may contribute to the change in the relevance of the synaptic input, e.g. activation of neighboring synaptic inputs that creates a dendritic spike, which has an increased probability of propagation to the soma, than an individual synaptic input (Segev and Rall, 1998).

The issue of coding strategies at neuronal level is still unresolved (Shadlen and Newsome, 1998, Softky, 1993, Softky, 1995), and it is also suggested that the two methods for coding information coexist or at least they are not mutually exclusive (Tsodyks and Markram, 1997). For example Singer and Gray (1995) proposed that features of an object in the visual field are coded in the firing rate of the neuron, however the different visual features of the same object are bound together by the synchronous activity of the neuronal assemblies representing the visual features. 


\section{EPSP-spike coupling in the cortical cells}

Calvin and Stevens (1968) have shown that, in spinal cord motoneurons, the main source of randomness in the interspike intervals originates from the fluctuating synaptic input.

Similarly, the irregularity of spike times in cortical neurons might be explained by the irregularly arriving synaptic inputs they receive. Stevens and Zador (1998) suggested that the irregularity of spike trains reflects temporal correlation among synaptic inputs (but see Destexhe et al., 2001).

Pyramidal neurons, which are the most common cells in the cortex (White, 1989), can generate very precise spike trains in response to rapidly fluctuating currents (Mainen and Sejnowski, 1995), showing that the spike generating mechanism is reliable. When cells from the visual cortex were stimulated with current waves containing combinations of lower and/or higher frequency sinus waves of the same amplitude that created fluctuations in the membrane potential, the cells behave differently in the presence of these frequencies. Lower frequencies were sufficient to depolarize the cells, only higher frequencies, which caused large fluctuations in the membrane potential, facilitated generation of spikes with high precision (Nowak et al., 1997). When the neuron was stimulated with a waveform that was generated from recordings of the membrane potential in vivo during visual stimulation and corrected for the filtering due to the capacitive and resistive properties of the neuron, produced a similar response in the term of reliability and precision than that of evoked with waveforms containing higher frequency sinus waves. Spike counts during visual stimulation were correlated with the magnitude of the high frequency components in the subthreshold membrane potential (Azouz and Gray, 1999b). Thus, these studies suggest that the kinetics of the individual synaptic inputs, which determines the frequency of fluctuation in the membrane potential, may be important for spike timing. 
Steady depolarization to membrane potentials close to spike threshold prolongs EPSPs in pyramidal cells (Stafstrom et al., 1984, Thomson et al., 1988, Stuart and Sakmann, 1995). The prolongation may lead to an imprecise timing of spike initiation. The balance between inward (mainly sodium) and outward (potassium) currents shifted toward the inward current as the cell membrane became more depolarized. The increased level of inward currents was attributed to the activation of persistent sodium currents (Deisz et al., 1991, Stuart and Sakmann, 1995, Fricker and Miles, 2000, Andreasen and Lambert, 1999), which has its threshold for activation near the firing threshold (Alzheimer et al., 1993). EPSP prolongation was not observed in interneurons (Fricker and Miles, 2000, Galarreta and Hestrin, 2001).

In summary, understanding the mechanisms underlying EPSP-spike coupling in cells with ongoing activity may provide an insight into the process of coding.

\section{Effect of network activity on EPSP-spike coupling}

In order to make predictions about EPSP-spike coupling in intact brains, we should consider that neurons are embedded in a complex neuronal network, and are receiving a continuous barrage of synaptic activity in vivo. Consequently, when neurons are more depolarized, the input resistance is reduced (Destexhe and Pare, 1999) and their membrane potential is continuously fluctuating (Azouz and Gray, 1999a, Lampl et al., 1999) compared to a neuron in vitro.

Pare and co-workers (Pare et al., 1998, Destexhe and Pare, 1999) applied TTX by microinjection within the cortex and monitored the changes in the input resistance of pyramidal cells in vivo using sharp electrode recordings. TTX inhibited spiking in the presynaptic cells and 
in this way it prevented the synaptic activity on the postsynaptic cell. As a result, it dampened the fluctuations in the membrane potential and produced a $30-70 \%$ increase in the cells' input resistance. In contrast, in slice preparations, where the level of spontaneous spiking activity is low, the effect of TTX on input resistance was insignificant. They concluded, that the main source of conductance originates from the ongoing spike-related synaptic activity in the network.

In vivo whole cell recording from cortical cells also showed that conductance increases substantially during visual stimulation (Borg-Graham et al., 1998, Hirsch et al., 1998, Anderson et al., 2000). The purpose of these studies was to elucidate the role of inhibition in the operation of visual circuitry and determine how the inhibitory conductances are related to the tuning curves of the cell. There was a general agreement that conductance reaches its maximum with optimal stimulus features, resulting in an increase of $20-300 \%$ in the level of conductance compared to the values measured at the resting state before stimulation (Borg-Graham et al., 1998, Hirsch et al., 1998, Anderson et al., 2000). The occurrence of excitatory and inhibitory conductances overlapped in time and the presence of "push-pull" inhibition was also reported (that is when the excitation increased, the inhibition decreased) in simple cells (Anderson et al., 2000, Hirsch et al., 1998). The presence of inhibition was also reported to be prominent in all cells (BorgGraham et al., 1998, Hirsch et al., 1998, Monier et al., 2003, Anderson et al., 2000), which is in contrast with the report by Berman et al., (1991), which stated, that inhibitory conductances did not contribute substantially to the cell's conductance level even during optimal stimulation. Inhibitory and excitatory conductance seemed to share preferred orientation selectivity of the cell to the stimuli (Martinez et al., 2002, Monier et al., 2003). Inhibition also occurred in the case of nonoptimal orientation of the bars used for stimulation (Volgushev et al., 1993, Monier et al., 2003 but see Berman et al., 1991). The amount of conductance increase depended on receptive 
field properties (Anderson et al., 2000) and orientation tuning of inhibition also depended on the laminar position of the cells (Borg-Graham et al., 1998, Martinez et al., 2002). Results from these in vivo studies suggest that large amount of conductance originates from the synaptic inputs to the pyramidal cells and the balance between inhibitory and excitatory ongoing synaptic activity in cortical cells.

\section{Response variability and ongoing synaptic activity in cortical cells}

Repeated presentation of the same sensory stimuli evokes highly variable spiking activity (Heggelund and Albus, 1978, Tolhurst et al., 1981, Tolhurst et al., 1983, Schiller et al., 1976), but see (Mountcastle, 1969). There is also a high trial-to-trial variability in the recorded membrane potential (Azouz and Gray, 1999a, Arieli et al., 1996). For that reason it was a widely accepted view that only spike rates averaged over a longer time period are reproducible and that the timing of spikes in the recorded spike train is essentially random. However, some recent in vivo recording studies support the notion that timing of spikes can be meaningful. The data suggest that cortical cells can generate precisely repeated spike patterns. Visual stimulation with a dynamic pattern generated highly precise spike trains (Buracas et al., 1998, Bair and Koch, 1996, de Ruyter van Steveninck et al., 1997). Short segments of recurrent spike patterns were also reported for acoustic stimulation (Villa and Abeles, 1990) or from single unit activity collected in the forebrain (Frostig et al., 1990b). Another example for the importance of timing are the synfire chains that are precisely timed spikes from sets of neurons connected to fire in a

feed-forward manner: when neurons from the first pool fires action potential synchronously that elicits spiking in the second pool of neurons in the sequence after a certain, precisely set length 
of time. Synchronous spiking in the second pool is followed by spiking in the neurons of the third pool in the sequence, keeping the time window constant with a submillisecond precision. The neuron pools participating in the synfire chains are not necessary located anatomically close. Synfire chains were detected on several occasions during physiological conditions (Prut et al., 1998).

Several studies suggested that variability in the level of ongoing synaptic activity might affect spike timing. For example, fluctuation of the membrane potential and spike timing were correlated prior to and during the stimulus, suggesting that variability in response to a stimulus is related to the basal state of ongoing activity in the neuronal network before stimulation (Arieli et al., 1996, Azouz and Gray, 1999a). Another study reported that variability in subthreshold activity was reduced in cells that had strong inhibition for the visual stimulation (Monier et al., 2003). Variability in spike timing during a visual stimulus also depends on the dynamics of the stimulus presented. Transient (e.g. drifting edges) but not static stimuli evoke highly precise spike trains in V1 neurons (Mechler et al., 1998). Similar observations were reported from the H1 neuron, in the fly's visual system (Warzecha and Egelhaaf, 1999).

Taken together, these data suggest that the presence of background synaptic activity interacts with the stimulus to control the spike output of the cell. The level of synaptic activity changes during physiological stimulation, and this itself have an effect on the spiking behavior of the cell. 


\section{Functional consequences of ongoing synaptic activity}

Based on modeling studies, it was predicted that ongoing synaptic activity could have a profound impact on synaptic integration (Bernander et al., 1991, Tanabe et al., 1999, Ho and Destexhe, 2000). Bernander et al., (1991) studied the effect of conductance originating from ongoing synaptic activity using a morphologically reconstructed pyramidal cell with passive dendritic processes receiving 4000 excitatory and 1000 inhibitory inputs. The background synaptic activity generated spontaneous firing and consequently reduced the membrane time constant. Hence, the window for synaptic integration time became much shorter and at the same time, the electrotonic distance from the soma to the distal part of the cell increased. The "strength" of the connection between the soma and dendrite also depended on the kinetics of membrane fluctuation and the cell's membrane potential. In the presence of the ongoing synaptic activity a large number of coincidentally activated synapses were necessary to generate a spike, which was estimated to be on the order of 100-1000 (Destexhe and Pare, 1999, Bernander et al., 1991, Segev and Rall, 1998). The shorter integration time also allowed the cell to discriminate better between synchronized or asynchronized inputs.

When voltage dependent conductances, such as $\mathrm{Ca}^{++}$or $\mathrm{Na}^{+}$-channels, or NMDA receptors were inserted in the dendrites of a similar model cell with ongoing spiking activity, those voltage dependent conductances amplified distal synaptic input locally, reducing the difference between the distal and proximal synaptic inputs (Bernander et al., 1994).

Depending on the experimental conditions, it was concluded, that increasing the level of background synaptic activity could result in a reduced precision (Cope et al., 1987) or reduced efficacy (Poliakov et al., 1996) in EPSP-spike coupling (defined as a probability of discharge due 
to the EPSP) compared to lower levels of synaptic activity. In contrast, theoretical studies concluded that network activity might improve responsiveness (Ho and Destexhe, 2000, Rudolph and Destexhe, 2001b, Destexhe et al., 2001, Rudolph and Destexhe, 2001a), or precision of spike timing (Pei et al., 1996, Tanabe et al., 1999) to subthreshold inputs.

In the theoretical models by Pei et al. and Tanabe et al. (Pei et al., 1996, Tanabe et al., 1999) the expression "noise" included two components: random background activity, and the possible noise originating from the spike generating mechanism (Gutfreund et al., 1995). For example, Tanabe et al., (1999) connected single compartments, where the behaviors of the compartments were described by Hodgkin-Huxley equations containing $\mathrm{Na}^{+}-$and $\mathrm{K}^{+}$- currents. Noise was introduced in this system by randomly setting the values of state variables of Hodgkin-Huxley equations of the compartments and the "cell" characterized by the distribution of resulting membrane potentials of the compartments. With the introduction of small amplitude noise, the average membrane potential was close to the resting potential and had a Gaussian distribution. Although the net effect of the low level noise was the depolarization of the cell, this depolarization was not large enough to create a higher frequency of discharge. When large amplitude noise was introduced, the membrane potential spent a significant time hyperpolarized to the resting potential as well as at a more depolarized membrane potential. Increasing the level of noise creates more excitation, which is reflected in higher frequency of firing: the response of the neuron to suprathreshold stimuli is still enhanced. When the fluctuation was increased further, it no only increased the probability of firing, but also the probability that a synaptic input activates during the relative refractory period of a spike. Thus, an increase in spiking probability may antagonize the response to a stimulus. They concluded that there should be an optimal level 
of noise to create spike trans to transmit information. The same model similarly explains the increase of the precision of spike timing for suprathreshold stimulus (Tanabe et al., 1999).

\section{Rationale of the study}

The neurons fundamental property is how synaptic inputs are integrated and generate spike outputs. Although the importance of spike timing in the coding of temporal information is unknown, there are arguments supporting both rate coding and temporal coding. Spike generation mechanism itself could be very precise and fluctuation in the membrane potential, which may originate from the synaptic activity of the presynaptic neurons, may facilitate precision of spike timing. Our knowledge on EPSP-spike coupling based on studies made under in vitro conditions, when the synaptic activity in pyramidal cells is low, unlike under in vivo conditions. According to modeling studies ongoing activity might influence the integration time window of EPSP-spike coupling. It was observed that at near threshold membrane potentials, voltage dependent conductances prolong the EPSP and the same conductances might affect how the cell responds to temporal information in its spike output.

In the slice, cells receive their synaptic input in a low rate, and as a consequence, they are silent. This condition however provides a better experimental control for the investigator over the stimulation parameters. In order to study the mechanism of EPSP-spike coupling at layer 5 cortical pyramidal cells in vitro, we mimicked the in vivo synaptic activity in the cell by injection of conductance waveforms using a dynamic clamp system (Sharp et al., 1993).

There are several advantages to using the dynamic clamp. It allows studying, how the presence of conductance might interact with both the passive and active properties of the cell. It 
also allows manipulating independently the level of conductance in the background activity. Two aspects of EPSP-spike coupling modulation were studied, the precision of spike timing, and the efficiency of EPSP-spike coupling. We also considered the effect of a single EPSP on the timing of spikes in a spike train. 


\section{CHAPTER II. EXPERIMENTAL METHODS}

\section{Cortical slices}

Animal care and usage for experimental purposes was in accordance with National Institutes of Health and Institutional protocols (http://www.nap.edu/readingroom/books/labrats/ chaps.html). 18-28 day old Sprague-Dawley rats were deeply anaesthetized with a mixture of ketamin-xylasine (80 $\mathrm{mg}$ and $6 \mathrm{mg} / \mathrm{kg}$ body weight, respectively). In some experiments mouse cells were used. In these cases 20-26 day old mice were anaesthetized with ketamine-xylazine mixture of $80 \mathrm{mg}$ and $16 \mathrm{mg} /$ body weight, respectively. When the animal did not show any response to a pinch in its tail, it was decapitated and the brain was quickly removed and placed into ice-cold oxygenated artificial cerebrospinal fluid (ACSF). ACSF contained (in mM) 125 $\mathrm{NaCl}, 2.5 \mathrm{KCl}, 1.25 \mathrm{NaH}_{2} \mathrm{PO}_{4}, 1 \mathrm{MgSO}_{4}, 2 \mathrm{CaCl}_{2}, 26 \mathrm{NaHCO}_{3}, 20$ glucose, 0.4 ascorbic acid, 1.2 pyruvic acid and 4 lactic acid. The ACSF was bubbled with a mixture of $95 \%$ oxygen and $5 \% \mathrm{CO}_{2}$ at $\mathrm{pH}=7.4$ during the dissection, incubation period and experiments. The hemisected brain was glued onto the stage of vibrating microtome (Leica, Nussloch, Germany), which had an angle of $30^{\circ}$, and parasaggittal slices with a thickness of $300 \mu \mathrm{m}$ were cut. After the dissection, the slices were incubated in a holding chamber at a temperature of $34^{\circ} \mathrm{C}$ for 30 minutes, and then stored at room temperature until recording. The recording temperature was 34$36^{\circ} \mathrm{C}$. Slices were transferred to the recording chamber and observed under an upright Zeiss microscope (Carl Zeiss Inc., Thornwood, NY, USA) equipped with a 40x water immersion lens, infrared differential interference contrast and a video camera (Cohu, Inc. San Diego, CA, USA) with a monitor. 


\section{Selection criteria for recording from layer 5 pyramidal cells}

Layer 5 pyramidal cells (Figure 1A) from the somatosensory or visual (2) cortex were identified visually with infrared microscopy and neurons were selected by their morphological properties and electrophysiological responses to hyperpolarizing and depolarizing step current

(McCormick et al., 1985). Cells were selected only from those slices that showed the presence of pyramidal cell dendrites running parallel with the surface of the slice. The same procedure was used both for rat and mouse cells.

To establish homogeneity in the sample cell population, input resistance, spiking pattern, spike properties were assessed. In case of mouse cells, the presence and amount of depolarization-induced prolongation of EPSP (see later) were also tested at the beginning of the experiments from $\mathrm{N}=10$ cells. However using the selection criteria above, no cells in mice were found that did not have the depolarization-induced prolongation of EPSP (measured as normalized EPSP half width, when cell was depolarized with a current step) and we did not find significant differences in this property between mouse and rat cells $(\mathrm{p}>0.1, t$-test for independent samples, $\mathrm{N}=15$ cells for rats, $\mathrm{N}=10$ cells for mice). Therefore the cells were pooled in the experiments. Similarly the values from the visual cortex were in the range the values measured from the somatosensory cortex of the rat, data from every cell were pooled.

Input resistance values were 22-62 MOhms measured at resting potential by injection of current step (100 pA or $-100 \mathrm{pA})$. Other selection criteria for including the cells in the sample populations were: (1) Resting potential value less than $-60 \mathrm{mV}$, which where not changed more than $5 \mathrm{mV}$ throughout the experiment, (2) Spike has an overshoot of $0 \mathrm{mV}$ throughout the experiment, (3) Membrane input resistance values did not change more than $10 \%$ during the 
A Pial surface

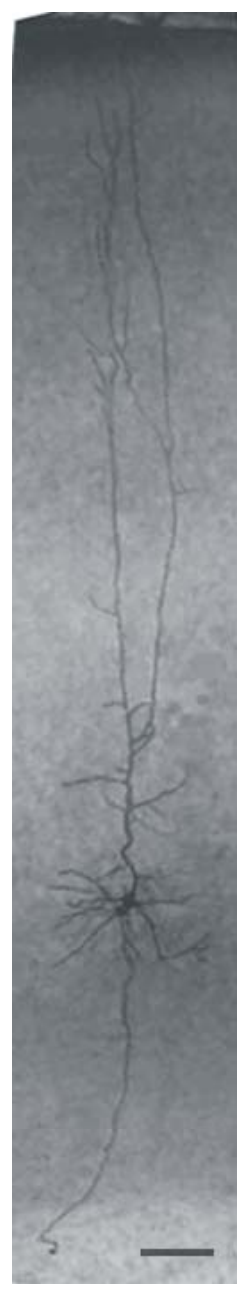

White matter
B

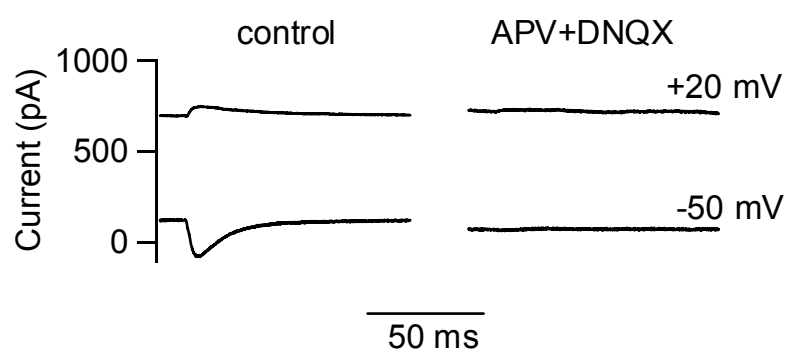

C
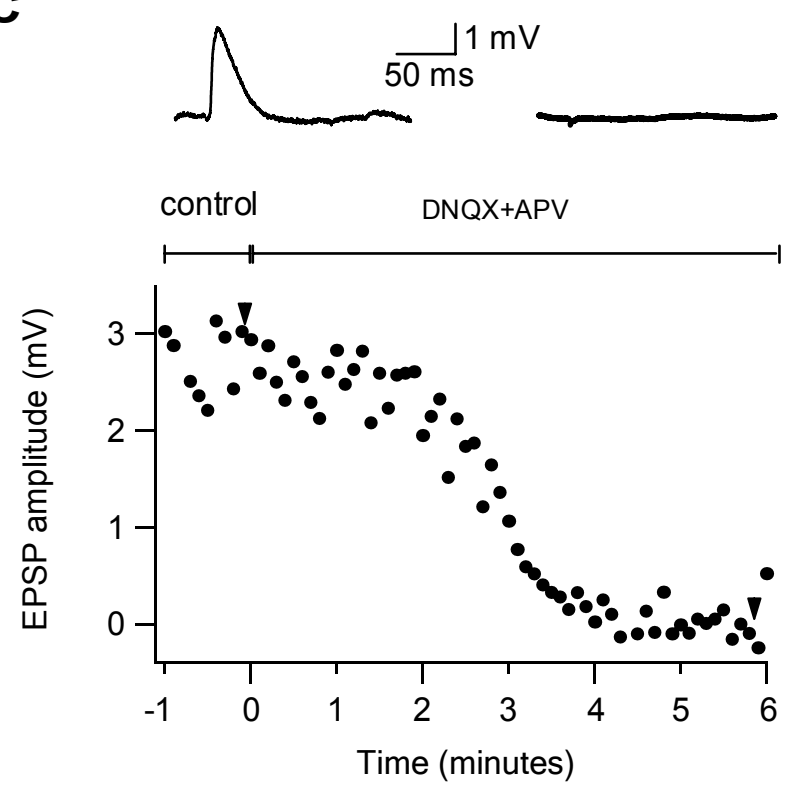

Figure 1. Evoked EPSP in the cortex. (A) Biocytin filled (see later in Experimental Methods) layer 5 pyramidal cell from the somatosensory cortex. Scale bar is $100 \mu \mathrm{m}$. (B) Mixture of APV and DNQX inhibits evoked EPSC. EPSC was measured under voltage clamp conditions at -50 and $+20 \mathrm{mV}$. Application of APV and DNQX at a concentration of $100 \mu \mathrm{M}$ and $10 \mu \mathrm{M}$, respectively, prevented the inward current at $-50 \mathrm{mV}$ and the outward current at $20 \mathrm{mV}$ in Csgluconate internal. (C) Evoked EPSP from a separate experiment, recorded in current clamp with the standard internal before and during the application of DNQX and APV. Arrows correspond to traces on upper panel. 
experiments (4) Experiments with a series resistance higher than $25 \mathrm{MOhm}$ were not included. Experimental data were not compensated for either series resistance or junction potential.

\section{Data acquisition}

Patch electrodes (2-4 MOhm) were filled with an internal solution containing (in $\mathrm{mM}$ ) $130 \mathrm{~K}$-methylsulphate, $4 \mathrm{KCl}, 10$ 4-(2-hydroxyethyl)-1-piperazineethanesulfonic acid (HEPES), 4 MgATP, 20 sodium-phosphocreatine, $0.3 \mathrm{NaGTP}, 0.2$ EGTA. Osmolarity of the internal solution was $295 \mathrm{mOsmol}$ at $\mathrm{pH}=7.3$. In some experiments $6.3 \mathrm{mM} \mathrm{KCl}$ was used. Except where otherwise noted, the internal solution above was used in all of the experiments, and it is referred as standard internal solution in the text.

The data acquisition system utilized an ITC-18 digitizing board (InstruTECH Co, Port Washington, NY, USA) as an interface between the amplifiers and the computer. The amplifiers were an Axopatch 200B and an Axoclamp 2A (Axon Instruments, Union City, CA, USA).

Dynamic clamp experiments were performed with either single or double electrodes.

When two electrode recordings where made, one electrode was used for data acquisition and the other electrode injected the current. Double electrode recording was preferred during the experiments and it was always used, whenever EPSPs were generated by injection of current (simEPSPs). The reason for using double electrode recording was to eliminate errors in measuring membrane potentials related to pipette the series resistance. Most of the experiments with evoked EPSPs, however, were made with using single electrode for dynamic clamp, because of the use of a third electrode for the inhibition of GABA receptors (see below). 


\section{Stimulation}

In order to characterize EPSP-spike coupling, we wanted to generate a monosynaptic EPSP. There is an advantage of evoking EPSPs by extracellular stimulation over evoking EPSPs by current injection, because the effect of reversal potential is considered, thus the level of current flow is not constant. The closer the membrane potential to the reversal potential to the ions related to synaptic activity, the less amount of current is generated. Another advantage is that extracellular stimulation in different layers results in EPSPs with time courses related to the dendritic location and receptor composition of the synapses.

Excitatory synapses are anatomically more prevalent in the cortex than inhibitory ones (White, 1989). However extracellular stimulation of afferent fibers elicits multiphase responses with an excitatory component mixed with a fast and a slow inhibition. The most common source of fast inhibition is $\mathrm{GABA}_{\mathrm{A}}$-receptor mediated, but applying $\mathrm{GABA}_{\mathrm{A}}$-receptor blockers in the bath is not a feasible solution to block GABA receptors, because by changing the balance between excitation and inhibition, the recurrent excitatory activation leads to epileptiform activity (Gutnick et al., 1982).

Evoked EPSPs (eEPSP) were characterized by their amplitude, half width and rise time. Half width was defined as the time between the rising and decay phase of the EPSP at half the amplitude. Rise time was defined as the time span during the rising phase of the EPSP between the $10 \%$ and $90 \%$ levels of the amplitude.

\section{$\underline{\text { Evoked EPSPs }}$}

Several different techniques are used to evoke monosynaptic EPSPs in the cortex. Stimulation of thalamocortical fibers (Agmon and Connors, 1991) in slices cut at a special angle 
or corticocortical stimulation on coronal slices (e.g., Kumar and Huguenard, 2001) yields a monosynaptic EPSP. A vertical cut from the deep layers to layer 1 and placing the stimulating electrode in layer 1 (Gonzalez-Burgos and Barrionuevo, 2001) also can generate monosynaptic EPSPs. GABA $A_{A}$ receptors can be blocked internally by picrotoxin, by 4,4 '-

Diisothiocyanostilbene-2,2'-Disulfonic Acid, or by eliminating ATP and GTP from the internal solution e.g. in (Nelson et al., 1994).

After reviewing and eliminating the possibilities discussed above, we developed a method based on Feldman (2000) and Castro-Alamancos et al. (1995) to evoke a monosynaptic EPSPs with focal application of a GABA-receptor antagonist, near the stimulating electrode. We used a theta-type glass electrode for extracellular stimulation. The tip of the theta electrode was broken to yield a tip diameter of 3-7 $\mu \mathrm{m}$ and filled with the ACSF used in the bath. A pair of Teflon coated platinum wires were stripped at the ends that interfaced with the ACSF, and were fitted into the holes of the theta electrode. These were then used for local, bipolar electrical stimulation. Stimulation was controlled by an optically isolated stimulation unit (DS2A Isolated Stimulator, Digitimer LTD., Welwiyn Garden City, UK). The electrode was positioned along the apical dendrite, 50-850 $\mu \mathrm{m}$ from the soma, and 10-100 $\mu \mathrm{m}$ from the apical dendrite horizontally. Pulses of 20-500 $\mu$ s were used for electrical stimulation. These had an amplitude of 30-75 V. The distance between recording and stimulating electrodes was estimated from the video monitor, which we had previously calibrated for distance measurements. GABA receptor blockers were dissolved in ACSF and applied by pressure near the stimulating electrode using a third pipette, or the from the stimulating theta electrode itself. $\mathrm{GABA}_{\mathrm{A}}$ and $\mathrm{GABA}_{\mathrm{B}}$ receptors were blocked by picrotoxin and CGP 55845 at a concentration of $1 \mathrm{mM}$ and $500 \mu \mathrm{M}$, respectively. 
The conditions necessary to evoke a monosynaptic EPSP in the cortex were tested and improved with voltage clamp recording, using a Cs-gluconate internal solution. The Csgluconate internal solution contained (in mM) gluconic acid 120-130, CsCl 6.3, HEPES 10, EGTA 10, MgATP 4, sodium-phosphocreatine 20, NaGTP 0.3. The pH was set at 7.3 with $\mathrm{CsOH}$, and the osmolarity was 290-295 mOsmol.

The majority of excitatory transmission in the cortex involves ionotropic glutamate receptors (Seeburg, 1993). Under our experimental conditions these receptors have a reversal potential around $0 \mathrm{mV}$, hence the monosynaptic EPSCs is an inward current at negative holding potentials and an outward currents at positive to $0 \mathrm{mV}$. We tested the presence of inhibitory synaptic activation during extracellular stimulation with application of a mixture of the ionotropic glutamate receptor antagonists, APV and DNQX, at concentrations of $100 \mu \mathrm{M}$ and 10 $\mu \mathrm{M}$, respectively (Figure 1B). These antagonists eliminated both the inward current at $-50 \mathrm{mV}$ and the outward current at $+20 \mathrm{mV}$ (Figure 1) related to the activation of the synapse. Had inhibitory conductances been present they would have created an inward current at holding potentials positive to $0 \mathrm{mV}$ in a solution containing glutamate receptor antagonists. We concluded from these experimental conditions were suitable to evoke a monosynaptic EPSP. Presence of inhibition was also tested after individual experiments made in current clamp (Figure 1C). Experiments with polysynaptic activity were discarded. An EPSP was considered polysynaptic, when either its rise time or decay time could not have been described as a smooth curve at resting potential.

EPSPs with amplitudes of 0.6-6.0 mV were evoked by extracellular stimulation for studying EPSP-spike coupling. Evoked EPSPs had a rise time of 1.6-7.7 ms, and half width of 15.6-37.0 ms at resting potential (Figure 2). 

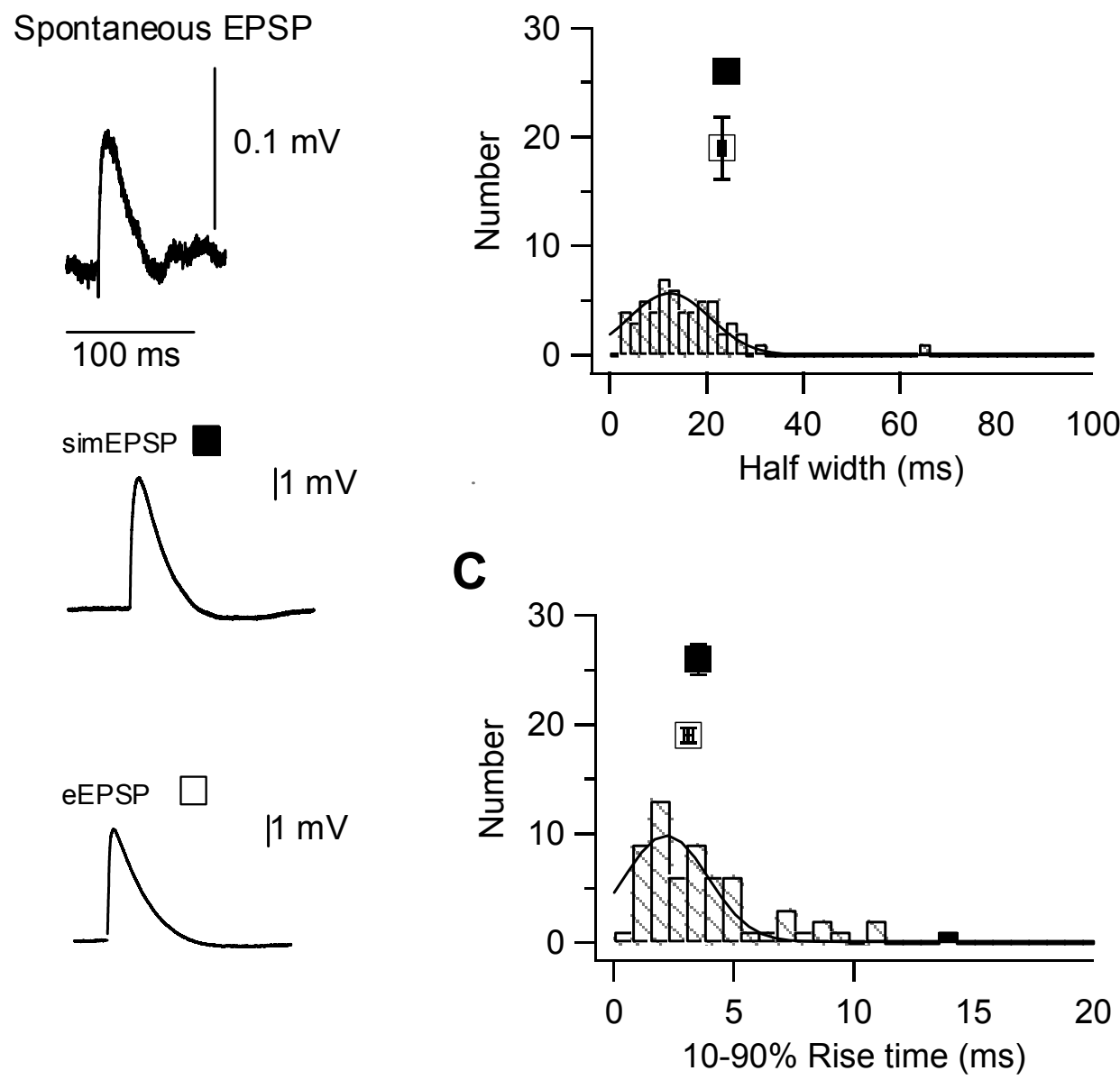

Figure 2. Comparison of spontaneous, simulated and evoked EPSPs on layer 5 pyramidal cells. (A) Spontaneous EPSPs $(\mathrm{N}=62)$ characterized with monotonically increasing rise phases and monotonically decreasing decays were collected from $\mathrm{N}=21$ traces of a single cell. EPSPs were selected visually and parameters were measured after a boxcar filter smoothing (see later in Data analysis). The distribution of half widths (B) and rise time constants (C) of these spontaneous EPSPs are shown with bars and the distributions fitted with Gaussian curves. Simulated EPSPs (simEPSP; closed squares) were generated in $\mathrm{N}=19$ cells by injection of a double exponential shaped current transient with an amplitude of $400 \mathrm{pA}$, rise and decay time constant of 0.1 and $5 \mathrm{~ms}$, respectively. The evoked EPSP (eEPSP; open squares) was generated by extracellular stimulation along the apical dendrite at $100 \mu \mathrm{m}$ from the soma. In comparison to the half widths (B) and rise times (C) of the spontaneous EPSPs we also included the average half widths and rise times of the simEPSPs (marked with filled squares) and eEPSPs (marked with empty squares). Markers for eEPSP and simEPSP were placed at their sample sizes. The marker also shows the standard error of mean (horizontal whiskers) and the standard deviation of the sample (vertical whiskers). The average half width of the spontaneous EPSP was $15.3 \pm 0.2$ $\mathrm{ms}$ and average rise time of the spontaneous EPSP $4.5 \pm 0.09 \mathrm{~ms}$. 


\section{$\underline{\text { Simulated EPSPs }}$}

SimEPSPs were generated by injection of a double exponential shaped EPSC (simEPSC) (equation 1).

$$
i=i_{\max } *\left(1-\exp \left(-t / \tau_{r}\right)\right) \star \exp \left(-t / \tau_{d}\right)
$$

The parameters for the equation are $i_{\max }$, which is the maximal amplitude of the injected current, $\tau_{r}$ is the rise time constant, $\tau_{d}$ is the decay time constant and $t$ is time. In most of the experiments, the same parameters were used: $i_{\max }=400 \mathrm{pA}, t_{r}=0.1 \mathrm{~ms}, t_{d}=5 \mathrm{~ms}$. The resultant simEPSPs had amplitude of 2.2-5.2 mV, a half width range of 18.6-24.3 ms, and a rise time range of 1.7-4.2 ms at resting potential (Figure 2). Parameters of simEPSP were measured only from experiments with double electrode recording.

When properties of eEPSPs and simEPSPs were compared to the properties of spontaneous EPSPs in layer 5 pyramidal cells, it was found that half widths and rise times of simEPSPs and evoked EPSPs were in the range of the half-widths and rise-times of spontaneous EPSPs (Figure 2).

\section{Dynamic clamp}

The ITC-18 board used as an interface between the computer and amplifiers (Figure 3) had a built-in implementation of dynamic clamp using the equation (2),

$$
I_{o}(t)=G_{c} \cdot T_{c}(t)+G_{e} \cdot T_{e}(t) \cdot\left(E_{e}-V_{m}(t)\right)+G_{i} \cdot T_{i}(t) \cdot\left(E_{i}-V_{m}(t)\right)
$$




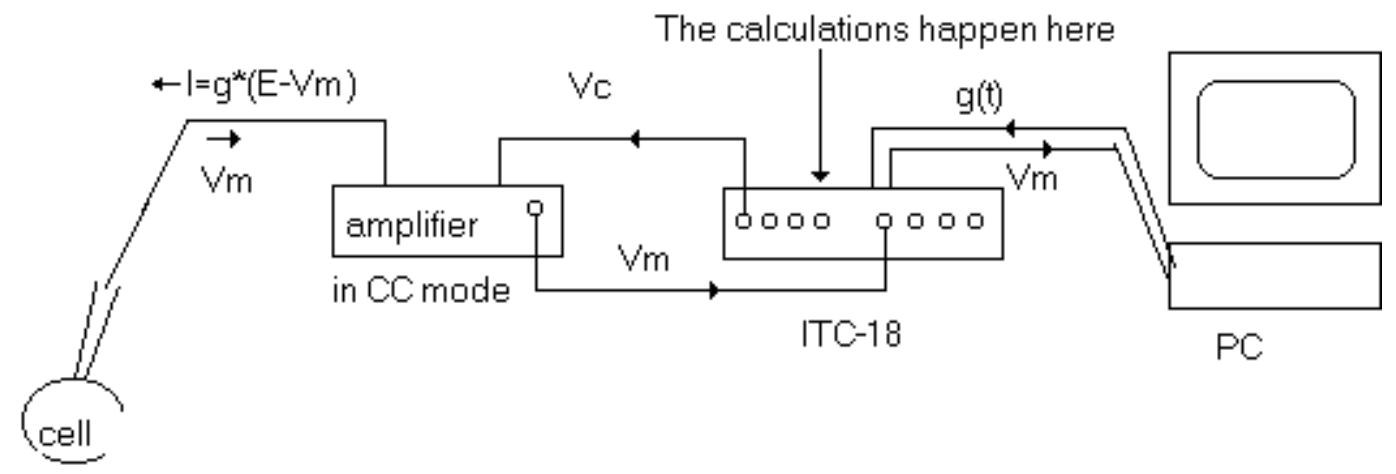

Figure 3. The data acquisition system used for dynamic clamp experiments. The computer was connected to an ITC-18 board and supplied the conductance values $(g(t))$. The board calculated the current using the membrane potential $\left(V_{m}\right)$ that were necessary to inject the conductance. The outgoing signal from the board was a voltage value $\left(V_{c}\right)$, which commanded the amplifier in current clamp mode to inject current. Note: The same system was used for voltage clamp and current clamp experiments. 
where $I_{0}(t)$ is the current injected into the cell in Amperes, $V_{m}(t)$ is the cell's membrane potential in Volts, $E_{e}$ is the reversal potential for excitatory conductances in Volts, $E_{i}$ is the the reversal potential for inhibitory conductances in Volts. In all experiments $E_{e}$ was set at $0 \mathrm{mV}$ and $E_{i}$ was $-60 \mathrm{mV} . T_{c}(t)$ is the forcing conductance for currents in Siemens (to include the constant current in equation (2) as a part of the linearization procedure, but practical value is 1 ; see below), $T_{e}(t)$ is the excitatory conductances in Siemens, $T_{i}(t)$ is the inhibitory conductances in Siemens, $G_{c}$ is the forcing gain in Volts, $G_{e}$ is the forcing gain for the excitatory conductance and $G_{i}$ is the forcing gain for the inhibitory conductance. Forcing gains $\left(G_{c}, G_{e}, G_{i}\right)$ are related to the voltage control of current output on the amplifier in current clamp mode. These numbers are derived from the amplifier gain in current clamp mode and the amplification of the digitalizing board. Inhibitory and excitatory conductances linearly added to an independent current injection $\left(T_{c}(t) * G_{c}\right)$ during the linearization procedure. According to equation (2), the conductances injected by the dynamic clamp does not depend on the membrane potential, similarly to the currents flowing trought AMPA and $\mathrm{GABA}_{\mathrm{A}}$ receptors. $T c(t), T e(t)$ and $T i(t)$ conductances were controlled by the computer.

The sampling frequency of $50 \mathrm{kHz}$ was fast enough to control conductance injection with the least chance for escape during fast signals, like action potentials. (Figure 4). The basic operation of the system was the following: in every sampling period $(0.02 \mathrm{~ms})$ the board collected one sample point of the membrane potential, multiplied with the voltage dependent parameters and offset it according to the voltage independent parameters in equation (1), then it injected the current into the cell. 
A1

injected excitatory current

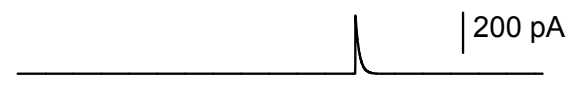

A2

desired conductance

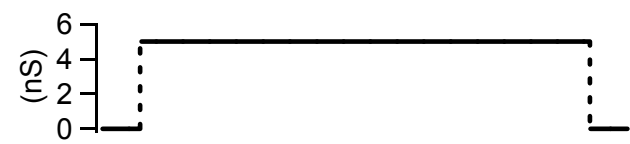

A3

measured voltage

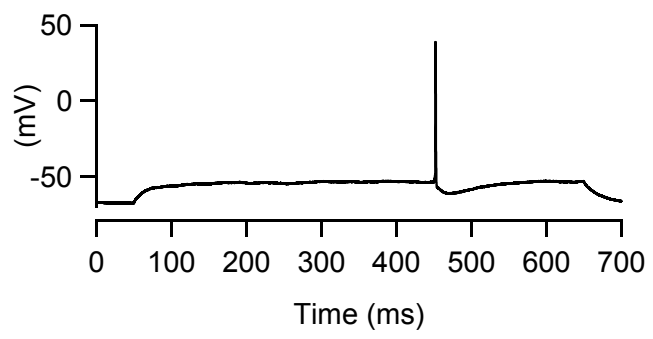

B1

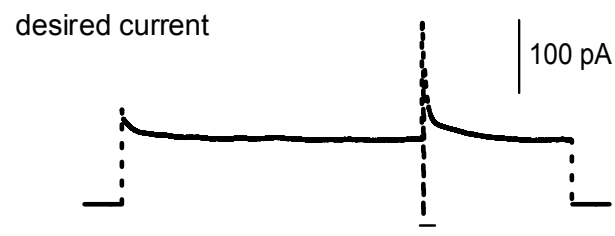

B2

measured current

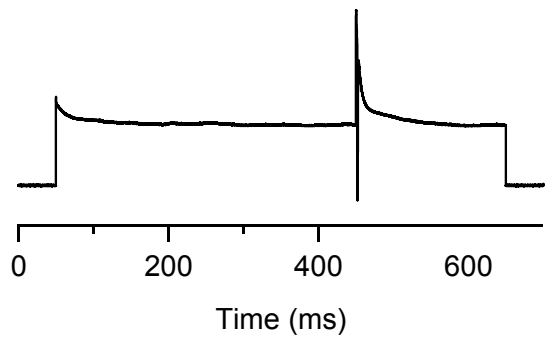

B3

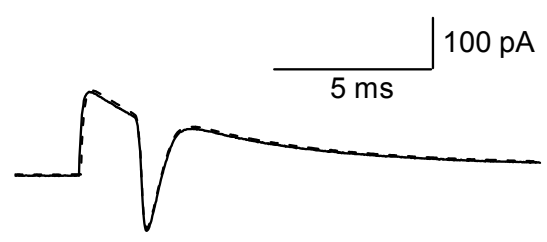

Figure 4. Test of the dynamic clamp. Dynamic clamp requires an accurate and continuous monitoring of the membrane potential and injection of current to generate the desired amount of conductance in the cell according to Ohm's law. The dynamic clamp system achieves this function in discrete steps: it acquires the membrane potential for a short period and injecting current. A fast change in the membrane potential may cause an oscillation in the membrane potential if the acquisition-current injection phase of the dynamic clamp system is not fast enough. To exclude this possibility and test how the dynamic clamp system controls the membrane conductance during spiking (A1-A3), we tested our system by evoking a spike (A3) by injecting an excitatory current transient (A1), while the cell was depolarized with conductance injection (A2). (B1) The desired current necessary to maintain the injected conductance according to I $=(\mathrm{V}-\mathrm{Erev}) * \mathrm{~g}$ equation. (B2) The time course of current was also measured during the experiment, which showed a good fit to the desired current on the expanded trace (B3). (B3) blown up version of traces on (B1) and (B2) marked by the bar. 


\section{Generation of background activity}

We simulated background synaptic activity using the dynamic clamp system. Unitary excitatory (Figure 5A1) and inhibitory current transients (Figure 5A2) were obtained from paired recording experiments between pyramidal cell presynaptic to another pyramidal cell and from fast spiking cell-pyramidal cell pairs, where the fast spiking cell was presynaptic to the pyramidal cell (Galarreta and Hestrin, 1998 and 1999). These unitary excitatory and inhibitory current transients from single traces were used to calculate conductance transients using Ohm's law and knowing the reversal potentials of the ions underlying the activation of excitatory and inhibitory synaptic activity (Figure 5A1 and 5A2 lower panels). The conductance transients were convolved with Poisson trains of $3000 \mathrm{~Hz}$ for the excitatory and $500 \mathrm{~Hz}$ for the inhibitory conductance waves (Figure 5B1 and 5B3, respectively), which generated fluctuations in the membrane potential (Figure 5B2) and spiking (inset). The injected conductance was offset by a current pulse $(-200$ to $+150 \mathrm{pA})$, or scaled to evoke a desired firing rate in the range of $0-30 \mathrm{~Hz}$. In some experiments step conductance was used instead of fluctuating conductance. When conductance was scaled, inhibitory and excitatory conductances were multiplied with the same number and injected together. The level of injected conductance varied between 2-50 nS and 1$25 \mathrm{nS}$ for the excitatory and inhibitory conductance levels, respectively. In most cases, the injected average conductance was between 20-30 nS for excitatory and 10-15 nS for inhibitory conductance. Generally, a step conductance of this value prevented the ongoing firing activity of the cell and allowed only spiking evoked by adding an extra EPSP (Figure 4). Fluctuating conductance however could evoke spikes even without an additional EPSP, when the cell was depolarized enough by the stimulation. 
A1

unitary EPSC

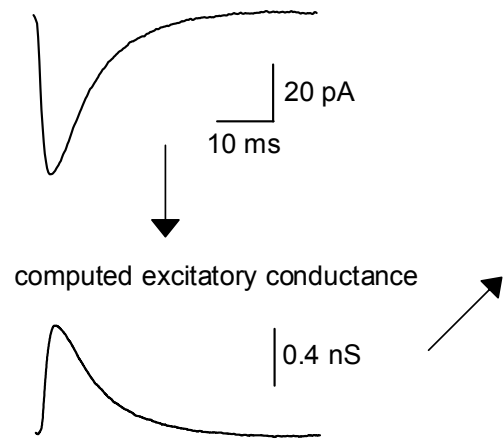

A2

unitary IPSC

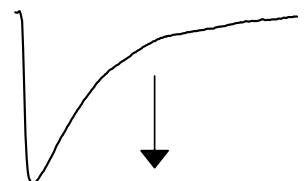

computed inhibitory conductance

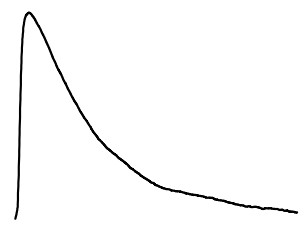

B1

excitatory conductance stimulation

B2
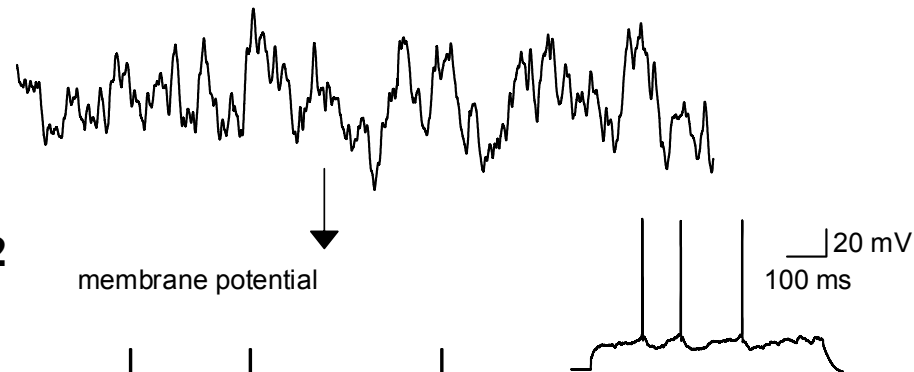

membrane potential

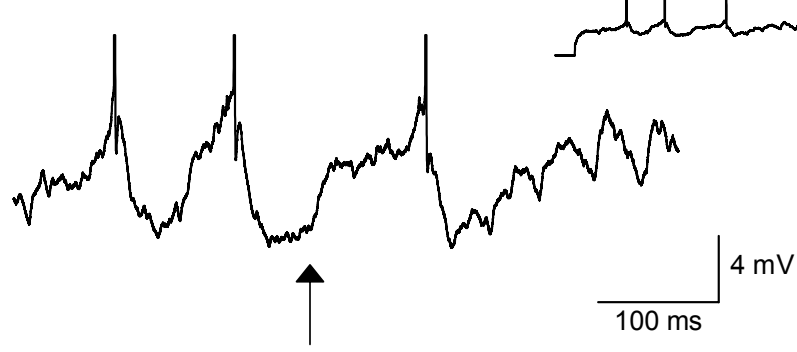

inhibitory conductance wave

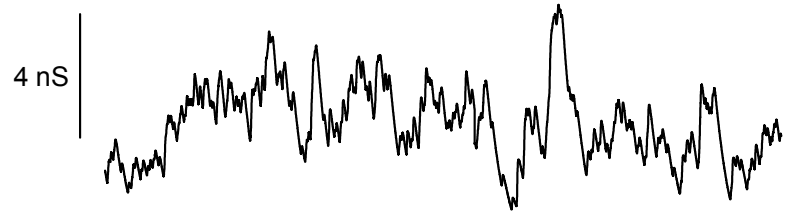

Figure 5. Generation of fluctuating background activity. (A) Unitary EPSC (A1 top) and IPSC (A2 top) from single traces were measured in paired recording experiment (Galarreta and Hestrin, 1998) and were used to calculate the unitary excitatory (A1 bottom) and inhibitory conductance transients (A2 bottom). (B) The conductance transients were convolved with Poisson-trains into excitatory $(3000 \mathrm{~Hz})$ and inhibitory $(500 \mathrm{~Hz})$ conductance waves $(\mathrm{B} 1$ and B3, respectively). Injection of these conductance waves using the dynamic clamp system generated a fluctuation in the membrane potential (B2) and initiated spiking activity (inset). 
In the experiments stimulated with fluctuating current, the stimulation was generated in a similar way to the fluctuating conductance stimulation. The average membrane potential was estimated to be $-50 \mathrm{mV}$ and the unitary conductance transients (Figure 5A1 and 5A2 top panels) were used to calculate putative unitary current transients at $-50 \mathrm{mV}$ using Ohm's law. The internal and external ionic concentrations were assumed to be equal to the ones used in the external and internal solutions during the experiments for the calculations. Then, these putative excitatory and inhibitory current transients were combined with the exact Poisson-train that were used to calculate excitatory and inhibitory conductance waves (e.g. in Figure 5B1 and B3) and the excitatory and inhibitory current waves they were summed and injected into the cell in current clamp mode.

\section{Data analysis}

\section{Comparison of data points}

Several hundred interleaving traces containing alternation of control traces and test traces were collected using a custom-made acquisition program. For the test traces only a single parameter was changed (e. g. the presence of an EPSP or the presence of conductance in the stimulation) in the stimulation protocol compared to the control traces in every experiment. Because of the simultaneous collection of data from a cell stimulated with these different experimental protocols, systematic changes in the cells' properties (see selection criteria) affected the response of the cells to the control and test stimulation in a similar way. Since changes in the EPSP properties under a given condition were related to the changes in the EPSP 
properties under a different condition, EPSP parameters originating from the same cells were compared by paired $t$-test.

Similarly in experiments, where the effects of fluctuating current and fluctuating conductance were measured on peristimulus time histogram (PSTH) parameters, data were collected in interleaving traces, allowing us to used the same paired $t$-test. In other cases, where the compared sample populations were generated from two different cell populations, independent $t$-test was used. These cases were mentioned in the text. Significance level was accepted at, when $\mathrm{p}<0.05$ and marked by “*”. Significance level $\mathrm{p}<0.01$ was marked with “**” and $\mathrm{p}<0.001$ was marked with “***”. Nonsignificant difference was marked with "ns". Data points are reported as means \pm standard errors of the mean.

\section{$\underline{\text { Calculation of peristimulus time histograms and cumulative sums }}$}

To analyze the effects of EPSPs on the discharge of the cell, PSTHs were generated from 70-400 traces. PSTH is a histogram of spike timing locked to the timing of the stimulus. The method is based on that spike trains are independent Poisson trains and the chosen bin size of the histograms is small enough to collect either 0 or 1 spike during a sweep (the probability of having a multiple spike in a bin during a sweep is 0 ). Spikes were collected into histograms with a bin size of $1 \mathrm{~ms}$. Spike number in every bin was normalized by the number of sweeps $(\mathrm{N})$ and the time span corresponding to the bin size. In this way the histogram shows the instantaneous frequency corresponding to the individual bins (Abeles, 1982, Perkel et al., 1967). The background firing frequency was calculated from instantaneous firing rate of those PSTH that were generated without the presence of EPSP, by averaging the bins from $200 \mathrm{~ms}$ from the starting point to the end of the stimulation. 
Experiments in these cases contained alternating traces of background stimulation only and EPSP superimposed on the same background stimulation. To follow how the probability of spike generation changes after an EPSP and measure the transient increases in the discharge frequency more accurately, cumulative histograms were calculated in the following way: Two PSTHs were generated from the alternating traces of background stimulation only and background stimulation with an EPSP superimposed on it. After subtraction of the first (PSTH made with stimulation only) from the second (PSTH made with stimulation and EPSP together), the difference was integrated. Peak synaptic gain was defined as the maximum on the cumulative sum histogram after the onset of the EPSP. The width of the peak synaptic gain was defined as a window between the 10 and $90 \%$ of the maximum on the cumulative sum.

\section{Auto renewal function}

After a spike a refractory period follows, that involves inactivation of sodium channels and activation of potassium conductances that hyperpolarizes the cell. The refractory period may manifests itself by reducing the probability of spiking for a stimulus for a longer period of time. For measuring how probability of spiking changes after a spike on cells with ongoing activity, auto renewal density functions (Perkel et al., 1967) were calculated (Figure 6). In every trace all the spikes were considered as reference points and the timing of spikes following each reference spike in a $100 \mathrm{~ms}$ window were measured and collected in a histogram with a bin size of $1 \mathrm{~ms}$. The time necessary to reach the half the average firing rate was assumed to be related to the refractory period of the cell. To quantify this window, a sigmoid curve was fitted (equation (3)) to the envelope of the histogram. The parameters are: $\max$ is the maximum of the function (the 

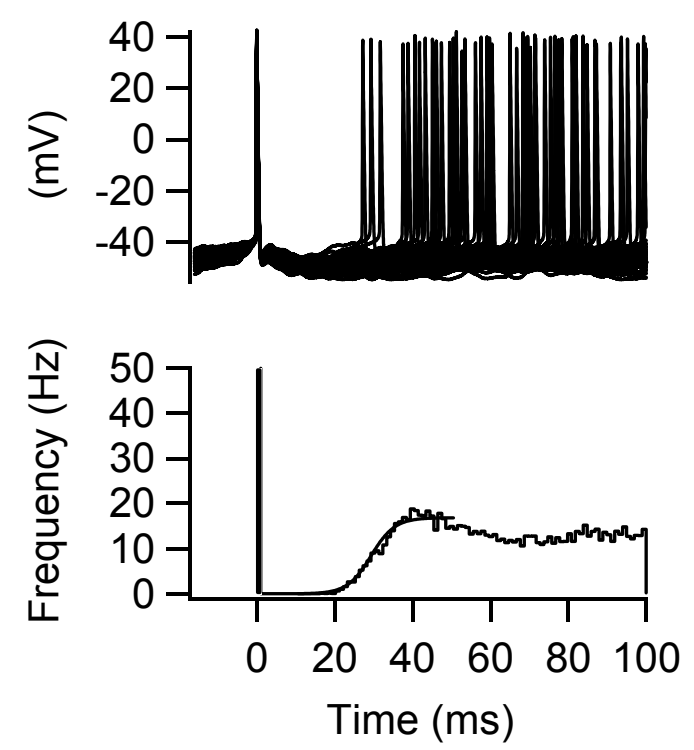

Figure 6. The renewal density function. Timing of every spike in the experiment was considered as a reference point (upper panel) and the timing of follower spikes were calculated in a $100 \mathrm{~ms}$ window. The spike timing was collected to a histogram with a $1 \mathrm{~ms}$ bin size. The beginning of the histogram was fitted by a sigmoid curve (solid line) and used to quantify the refractory period of the cell. 
asymptote, base is vertical offset from abscissa, in our condition was equaled 0 . Rate measured the rise time of the sigmoid curve and xhalf was the time window to reach the half the maximum.

$$
f(x)=\text { base+ } \frac{\max }{1+\exp ((\text { xhalf-x }) / \text { rate }}
$$

\title{
Filtering
}

Traces for collection of spontaneous EPSPs on Figure 2 were smoothed with a moving average filter, called boxcar filter, where 9 points before and after the smoothed value were averaged together with the smoothed value. Similar boxcar filtering was used for calculation of reliability and precision of spiking.

\begin{abstract}
Anatomy
Our purpose was to select pyramidal cells (see the selection criteria above), however we were also interested in if the chosen population is not homogenous, for example if the sample population contained interneurons. For this reason cells $(\mathrm{N}=10)$, chosen from the sample population randomly, were stained intracellularly by biocytin. $0.2 \%$ biocytin was added to the intracellular solution and diffused in the cell. After completion of the experiments, slices were fixed for 2-4 days at $0-4{ }^{\circ} \mathrm{C}$ in $4 \%$ paraformaldehyde dissolved in phosphate buffered saline (PBS) at $\mathrm{pH}=7.35-7.45$. At the beginning of the staining, slices were rinsed two times in PBS for 5 minutes, then again, for 10 minutes. Endogenous peroxidase activity was neutralized by incubating the slices in PBS containing 10\% methanol, $1 \% \mathrm{H}_{2} \mathrm{O}_{2}$ and $90 \%$ PBS for 10 minutes.
\end{abstract}


After the incubation period, slices were washed 3 times for 10 minutes in PBS. Cell membranes were permeabilized by washing the slices in a $2 \%$ Triton X-100 in PBS for 1 hour, then biocytin staining was processed by standard avidin-biotinylated-horseradish peroxidase complex (Vecstatin ABC-kit, Vector Laboratories, Burlingame, CA, USA). After a 2 hour incubation in the $\mathrm{ABC}$ (made from 2 drops of solution A, 2 drops of solution $\mathrm{B}$ added to a $1 \%$ Triton $\mathrm{X}-100$ solution), the slices were rinsed 2 times for 10 minutes, a third time for 15 minutes and a fourth time for 1 hour in PBS. 0.5\% 3-3' Diaminobenzidine (DAB) solution contained $3.34 \mu 130 \%$ $\mathrm{H}_{2} \mathrm{O}_{2}$ and $5 \mathrm{mg}$ DAB in $10 \mathrm{ml}$ PBS (Figure 1A). The reaction was followed under the microscope and it took about two minutes. After the reaction, slices were rinsed 3 times for 15 minutes in PBS and placed in $0.1 \%$ osmiumtetroxyde in PBS for about 2 minutes then, washed overnight in PBS.

The slices were then mounted on slides in Mowiol (Calbiochem, San Diego, CA, USA) containing mounting medium to prevent fading, covered with a cover slip, and left out to dry overnight. Slices were observed with a binocular microscope under 10x and 40x magnification. Picture on Figure 1A was taken with a CCD camera mounted on a Zeiss microscope (Axiophot, Zeiss Inc., Thornwood, NY, USA).

All the recovered $(\mathrm{N}=10)$ were layer 5 pyramidal cells. 


\section{CHAPTER III. RESULTS}

\section{The effect of conductance on the properties of the cell}

Injection of conductance in the soma of pyramidal cells affected the input resistance and membrane time constant of the cell. The reduction of the membrane input resistance by conductance was measured with a small $(50 \mathrm{pA})$ depolarizing or hyperpolarizing current at the maximal value of the voltage deflection. (Figure 7A). These experiments also confirmed that changes in the membrane input resistance can be estimated from the value of input resistance of the cell and the amount of conductance injected. In all the following experiments this reduction is reported as a percentage reduction in the value of input resistance measured at resting potential with no conductance injected in the cell. For example, if the overall input resistance was reduced by $60 \%$ that means that the membrane potential deflection due to the hyperpolarizing current was $40 \%$ of the original membrane potential deflection after the application of the conductance.

The membrane time constant was measured at resting potential by injection of $5 \mathrm{~ms}$ current pulses, whose amplitude was $-100 \mathrm{pA}$ (Fig. 7B). To restore the resting potential of the cell when conductance was present, a hyperpolarizing current step was also injected. The decay of the deflection in the membrane potential due to the current pulse was fitted with a single exponential curve. (Figure 7B). The membrane time constant was between 19-25 ms with no background conductance and the amount of conductance we injected in most of the experiment reduced it to 7-9 ms ( $\mathrm{N}=5$ cells). $20-30 \mathrm{nS}$ excitatory and 10-15 $\mathrm{nS}$ inhibitory conductance were injected in most of the experiments.

Spike properties were also affected by the conductance (Table 1.) 
A

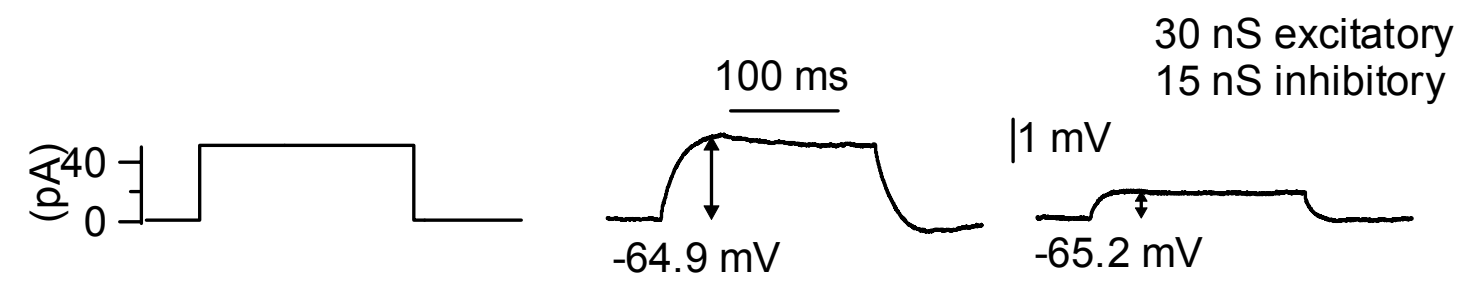

B
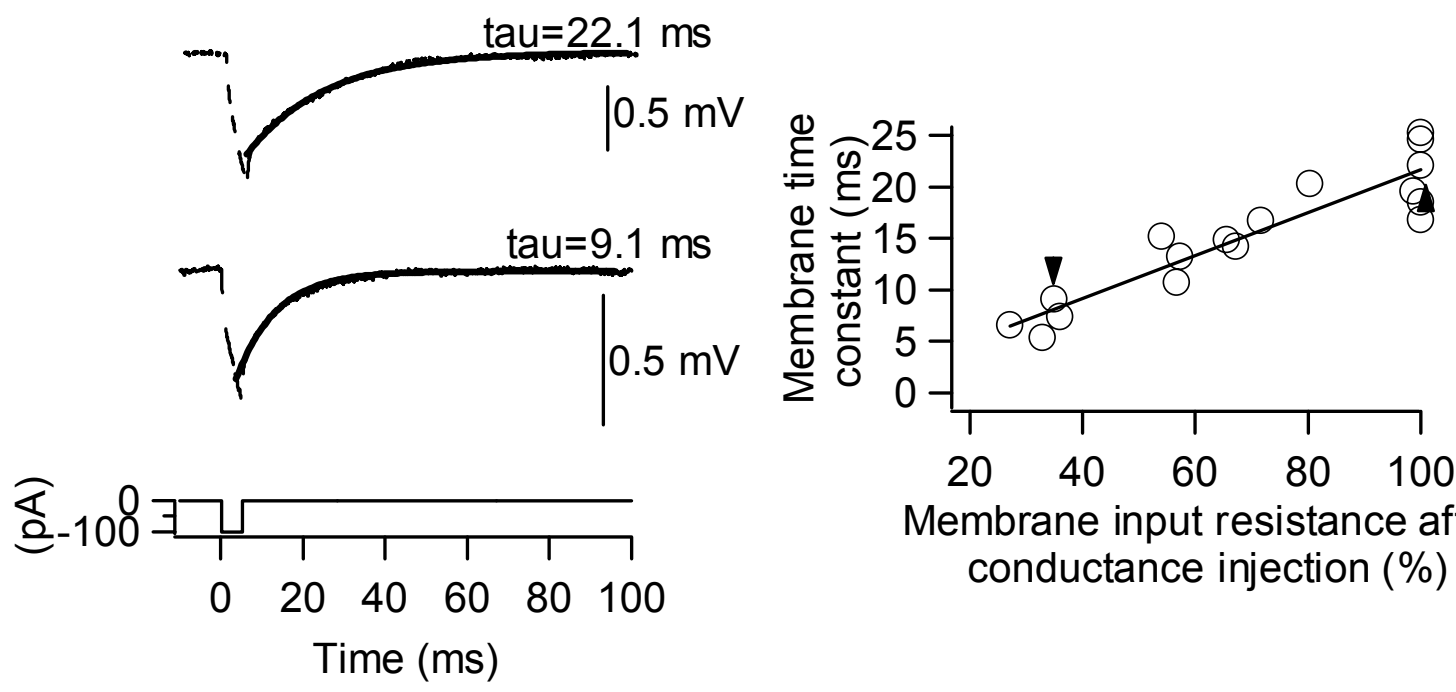

Membrane input resistance after conductance injection (\%)

Figure 7. Effect of injection of conductance on the membrane properties of the cell. (A) Injection of conductance reduces the membrane input resistance. Arrows show the points for measurement of the input resistance. (B) Injection of conductance reduces the membrane time constant. Sample traces on (A) and (B) are from the same cell. Membrane potential in response to an injection a $5 \mathrm{~ms},-100 \mathrm{pA}$ current pulse was fitted with a single exponential curve to characterize the membrane time constants (tau). Different levels of conductance were injected in the cells and all tau values from the exponential fits were pooled together. Measurements are from 5 cells and every cell contributes to at least 2 data points. Arrows depict actual measurements from a single cell (dashed lines) on first panel. 
Table 1. Spike properties and the presence of background conductance. Several number of spikes (at least 50 for every data point from every cell) were averaged from $\mathrm{N}=7$ cells under two conditions: when the cells were stimulated with fluctuating current (Current) or fluctuating conductance (Conductance). Stimulation was executed in interleaving traces, stimulation with fluctuating conductance was followed by stimulation with fluctuating current and vice versa. Data from experiments with double electrode recording (3) and single electrode recording (4) showed no difference, and all are pooled and included in the table. Spike amplitude was measured between the spike threshold and maximum of the spike. Half width was measured between the points defined the spike amplitude at $50 \%$ size between the rise phase and decay phase of the spike. The AHP was defined as the voltage difference between the threshold and the minimum of afterhyperpolarization after the spike. "*” marks significant differences tested with paired $t$-test $(\mathrm{p}<0.05)$.

\begin{tabular}{l|cc} 
& Current & Conductance \\
\hline Threshold $(\mathrm{mV})$ & $-41.6 \pm 1.62$ & $-42.7 \pm 1.25$ \\
Amplitude $(\mathrm{mV})^{*}$ & $83.8 \pm 2.55$ & $74.2 \pm 2.91$ \\
Half width $(\mathrm{ms})^{*}$ & $0.70 \pm 0.05$ & $0.62 \pm 0.04$ \\
AHP $(\mathrm{mV})^{*}$ & $-16.8 \pm 1.55$ & $-11.0 \pm 1.43$
\end{tabular}




\section{Conductance reduces depolarization induced prolongation of EPSP}

Depolarization to potentials near firing threshold in response to current injection results in an increase in the amplitude and prolongation of the half width of EPSPs in layer 5 pyramidal cells (Stafstrom et al., 1984, Deisz et al., 1991, Stuart and Sakmann, 1995, Thomson, 1997) and hippocampal pyramidal cells (Fricker and Miles, 2000). However under in vivo conditions, the depolarization is caused by synaptic conductances, and the interaction of the EPSPs with the background activity could influence the prolongation and increase in the amplitude of EPSPs. To test this hypothesis we evoked monosynaptic EPSPs with extracellular stimulation, while the cell was depolarized by injection of a conductance step (Figure 8). The level of conductance was chosen to reduce the input resistance of the cell by about $49-73 \%$ with an average of $63.1 \pm$ $3.1 \%(\mathrm{~N}=7$ cells $)$. Depolarized state was measured at close to firing threshold where probability of firing was about $50 \%$. The amount of reduction in the input resistance had a range that was reported in the cortical cells in vivo (Destexhe and Pare, 1999, Pare et al., 1998, Borg-Graham et al., 1998). To compare the EPSP properties at resting potential and at membrane potentials close to firing threshold, we injected the same conductance together with a hyperpolarizing current to restore the original resting potential. Half widths of eEPSPs had a range of 18.2-34.6 ms with an average of $26.5 \pm 2.3 \mathrm{~ms}(\mathrm{~N}=7$ cells $)$ at resting potential and depolarization near firing threshold by a current step increased this value 2.0-4.1 -fold. We defined normalized half width as the ratio of half widths measured at depolarized potentials and measured at resting potentials when the same amount of conductance were injected. The average normalized half width was of $3.0 \pm$ 0.3 ( $\mathrm{N}=7$ cells) with no conductance injected. However depolarization by a conductance step from resting membrane potential to near the firing threshold dramatically reduced the 
A2 Conductance
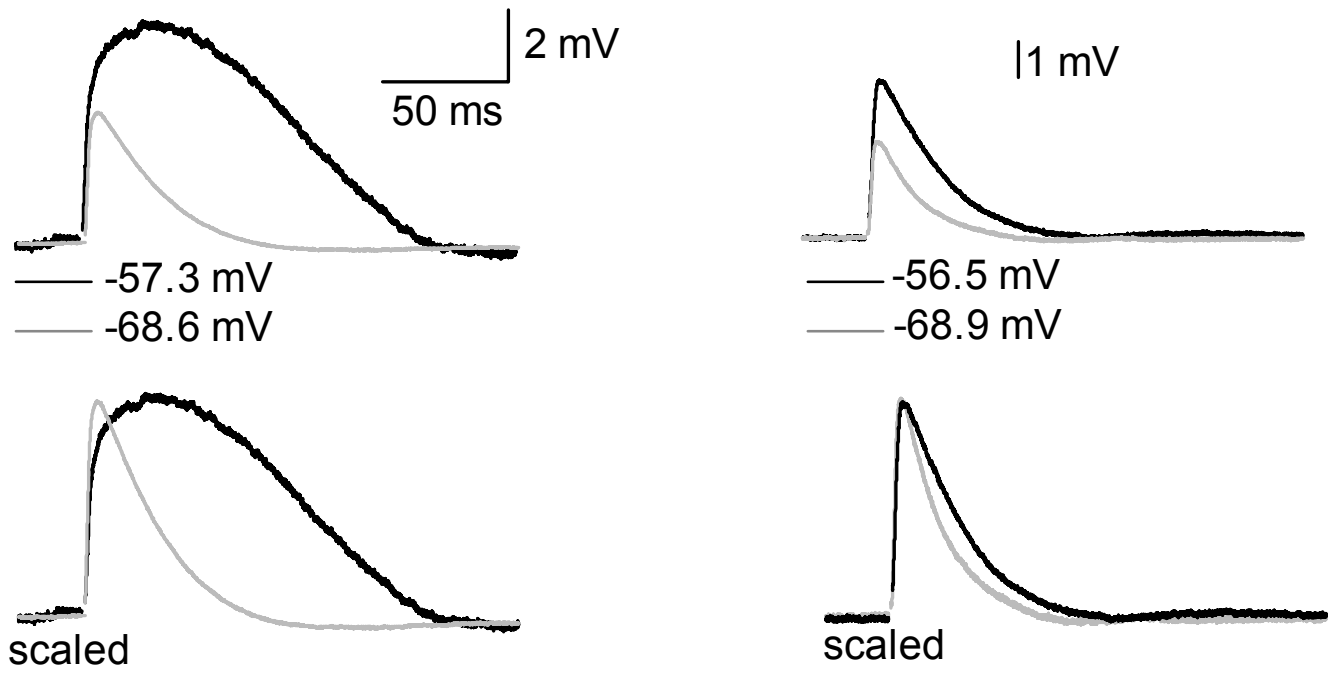

B
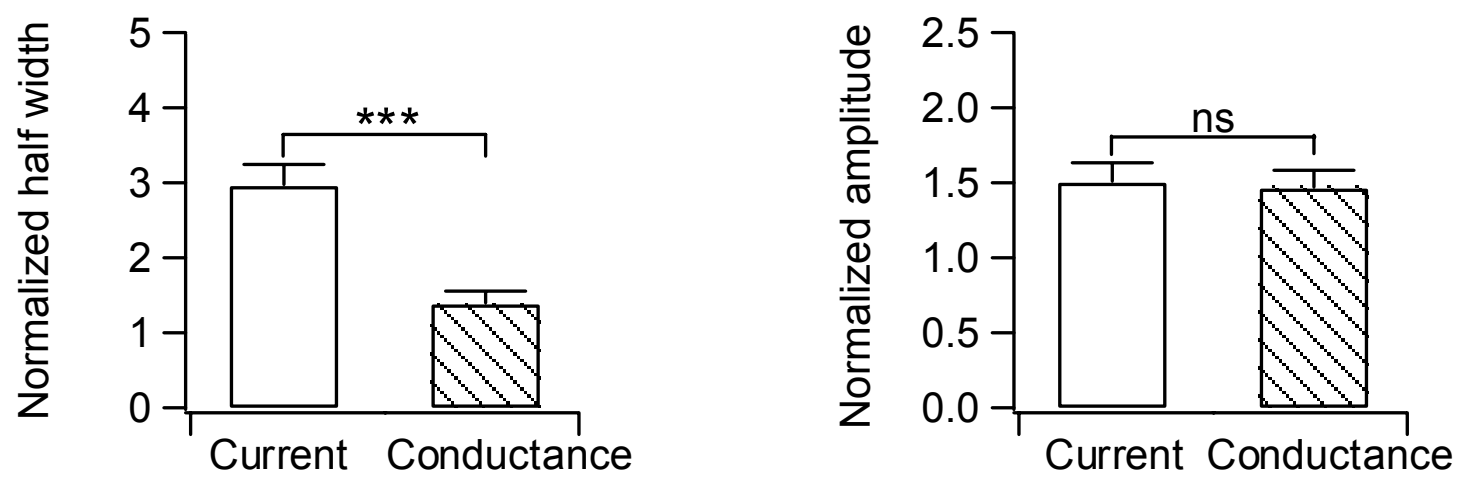

Figure 8. Effect of background synaptic conductance on the properties of eEPSP. (A) EPSPs with amplitude in the range of $0.6-3.1 \mathrm{mV}$ at resting potential were evoked by stimulation with a glass electrode placed $50-700 \mu \mathrm{m}$ from the soma along the apical dendrite. The cell was depolarized by a step current (A1) or a step conductance (A2). Averages from 3-50 traces. Note the scale difference between traces with current or conductance background on the upper panels. (B) Depolarization by conductance reduced the depolarization-induced prolongation of the EPSP $(\mathrm{N}=7)$. (C) Depolarization increased the amplitude of eEPSP under both conditions $(\mathrm{N}=7$ cells). 
prolongation of the EPSP half width and the normalized half width was $1.4 \pm 0.2$ on the same cells $(\mathrm{N}=7)$. The difference in the prolongation of the EPSP half width when the cell was depolarized with conductance or depolarized with a step current was significantly different $(\mathrm{p}=0.001, \mathrm{~N}=7$, Figure $8 \mathrm{~B})$.

Depolarization was also reported to increase the EPSP amplitude together with the prolongation of half width (Stuart and Sakmann, 1995, Fricker and Miles, 2000, Stafstrom et al., 1984, Thomson, 1997, Deisz et al., 1991). Because of the extra conductances, the amplitude of EPSP is expected to be reduced, when compared to the amplitude obtained without the presence of conductance (Figure 8A1 and A2). Amplitudes of the eEPSPs at resting potential values ranged from 0.6 to $3.6 \mathrm{mV}$ without the presence of conductance and they ranged from 0.3 to 2.7 $\mathrm{mV}$ with the presence of conductance on the same cells $(\mathrm{N}=7)$. We defined the normalized amplitude as a relative increase in the EPSP amplitude due to depolarization from the resting potential to membrane potential values close to firing threshold. When we measured the normalized amplitude there was no significant difference under the two conditions. The average value of the normalized amplitude was $1.5 \pm 0.1$ and $1.5 \pm 0.1$ for current and conductance, respectively ( $\mathrm{p}=0.773, \mathrm{~N}=7$ cells).

The previous experiment was repeated with simulated EPSPs (simEPSP) instead of eEPSPs. To evoke simEPSPs with similar amplitude with and without the presence of the background conductance, the size of the injected double exponential shaped current (simEPSC) was scaled to compensate for the effect of background conductance. The amplitude of the simEPSPs was in the range of 4.0-4.6 $\mathrm{mV}$ with an average of $4.2 \pm 0.1 \mathrm{mV}$ at the resting potential (Figure 9; $\mathrm{N}=9$ cells). The injected conductance reduced the input resistance in the 
A

Current

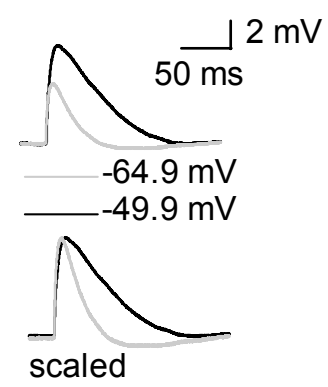

C

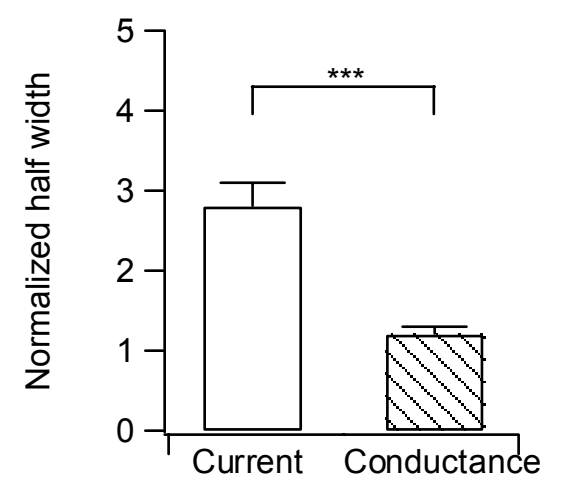

B

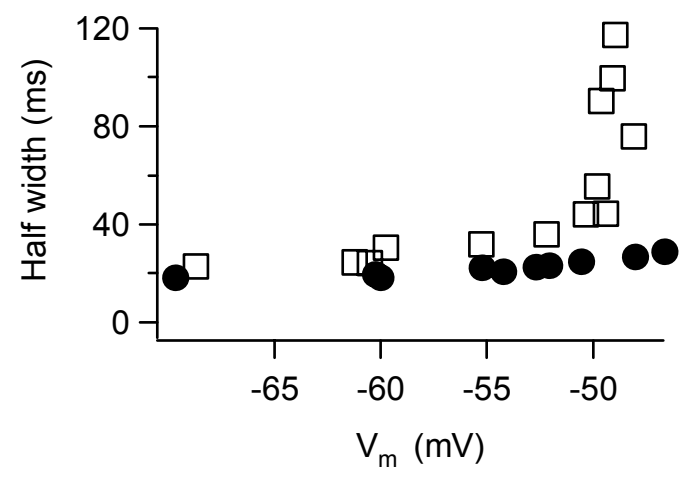

D

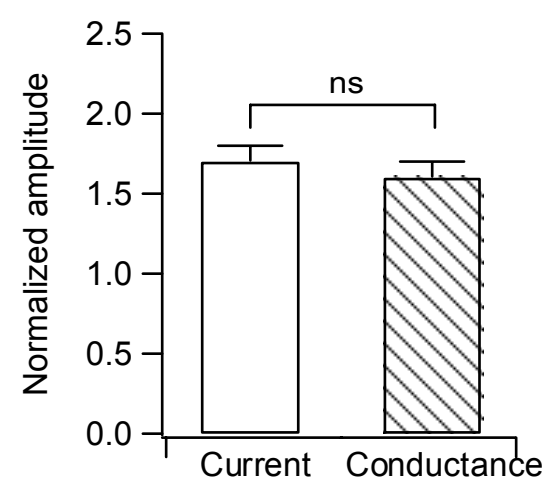

Figure 9. Effect of background synaptic conductance on the properties of simulated EPSP. (A) The experiments on Figure 8. were repeated with simEPSPs. The amplitude of the simEPSC was scaled to yield a simEPSP with similar size at resting potential with and without the background conductance. (B) Membrane potential determines the EPSP half width prolongation in a different way with (filled circles) and without (empty squares) conductance in the background. Same cell as on (A). (C) Effect of conductance on the EPSP half width at membrane potential close to firing threshold relative to that of at resting potential ( $N=9, p<0.001)$. (D) Amplitude of the EPSP with and without conductance $(\mathrm{N}=9, \mathrm{p}>0.117)$. 
range of 27.9-65.7 with an average of $48.4 \pm 5.3 \%$ ( $\mathrm{N}=9$ cells). Small changes in the membrane potential in the range close to firing threshold generated large differences in the prolongation in the simEPSP half width (Figure 9B). Stuart and Sakmann (1995) reported similar results. Depolarization by conductance changed the nature of this dependence (Figure 9B), resulting in a diminished prolongation of the EPSP half width near threshold membrane potentials.

The average of normalized half width (depolarized/rest) was of $2.8 \pm 0.3$ without the presence of conductance $($ range $=2.1-5.2, \mathrm{~N}=9$ ). Depolarization by a conductance step from resting membrane potential to near the firing threshold significantly reduced the prolongation of the simEPSP half width and the normalized half width became 1.2 \pm 0.1 -fold (Figure 9C; range $=0.9-1.6, \mathrm{p}<0.001, \mathrm{~N}=9$ cells $)$.

The increase in the simEPSP amplitudes due to the depolarization from the resting potential was not significantly different under the two conditions (Figure 9D; $p=0.117, \mathrm{~N}=9$ ), similarly to the eEPSP amplitudes. Depolarization also increased the rise time of the EPSPs, but this effect was reduced when background conductance was injected. We assessed with calculating the normalized EPSP rise time, which was defined as the ratio (depolarized/resting) of the EPSP rise time obtained at membrane potentials near spike threshold and at resting potentials. The normalized EPSP rise time was $1.6 \pm 0.2$ when the neuron was depolarized with current from resting potential. The presence of a background conductance reduced normalized EPSP rise time to $1.3 \pm 0.2$ The comparison showed that the background conductance significantly affected the rise time of the eEPSPs ( $\mathrm{p}=0.039, \mathrm{~N}=7$ cells). Normalized rise time of simEPSPs was $1.9 \pm 0.2$ (range: 1.3-2.7), when conductance was not present and it was reduced to $1.3 \pm 0.1$ with conductance in the background (The range was: $0.9-1.5$ ). This difference was also significant ( $\mathrm{p}=0.003, \mathrm{~N}=9$ cells $)$. 
There was no significant difference between the normalized half widths of evoked EPSPs and the normalized half widths of simulated EPSP obtained in the presence of conductance or between the ones, obtained without the presence of conductance $(\mathrm{p}=0.478$ with conductance and and $\mathrm{p}=0.188$ without conductance, respectively; $\mathrm{N}=7$ for eEPSP and $\mathrm{N}=9$ for $\operatorname{simEPSPs}$ ). Similarly, there were no significant differences in the samples of eEPSP and simEPSP for normalized amplitudes and normalized rise times either in the presence or in the absence of background conductance $(\mathrm{p}=0.214$ for normalized amplitude with the presence of conductance, $\mathrm{p}=774$ for normalized amplitude depolarized with a step current, $\mathrm{p}=0.883$ for normalized rise times in the presence of conductance and $\mathrm{p}=0.294$ for normalized rise times depolarized with a step current).

These results indicated that background conductance reduces EPSP prolongation related to membrane potential depolarization without affecting the increase of the EPSP amplitude in a similar way both on evoked and simulated EPSPs.

\section{Effect of membrane potential fluctuation and the amount of conductance on EPSP properties}

The previous experiments were carried out with a conductance step as a background activity, instead of fluctuating conductance. Ho and Destexhe (2000) suggest that it is important to study separately the effect of tonic conductance from the fluctuating membrane potential, because these two conditions could influence physiological parameters in an opposite manner. While conductance attenuates subthreshold activity, a fluctuation in the membrane potential might increase the sensitivity of the cell to subthreshold stimuli compared to the conditions with 
no fluctuation, resulting in higher probability of firing for stimuli of the same size. One explanation could be that tonic conductance simply affects the passive properties of cell. However, a fluctuation in the membrane potential may influence the activation and inactivation of voltage-dependent ionic channels, which in turn can amplify or reduce EPSP amplitude or half width. To test whether a fluctuation in the background activity elicits any additional change in the reduction of depolarization-induced prolongation of simEPSPs, the same amount of conductance was injected as a step or fluctuation $(20 \mathrm{nS}$ excitatory and $10 \mathrm{nS}$ inhibitory conductance, $\mathrm{N}=6$, Figure 10A and B). To obtain the parameters of the EPSP at resting potential, a hyperpolarizing current was injected while depolarizing with the step conductance or the fluctuating conductance wave. In the case of fluctuating conductance, traces with both background and a simEPSP was interleaved with traces in which only the background conductance was simulated. The same fluctuating conductance wave was used in every trace throughout the experiments (see Figure 5). To measure the properties of the simEPSP more reliably, background only traces were subtracted offline from the traces stimulated with simEPSP and background together, then these trace differences were averaged from 5-20 traces for every data points from each cell.

Membrane potential values were calculated as an average membrane potential of those traces stimulated with background conductance only during a $5 \mathrm{~ms}$ interval that was related to the first $5 \mathrm{~ms}$ of the simEPSP on traces stimulated with simEPSP superimposed on background activity. The $5 \mathrm{~ms}$ interval was chosen, because in most cases simEPSP rise times were smaller than $5 \mathrm{~ms}$, and background stimulation did not show abrupt changes during this short window. The amplitude of simEPSP increased by depolarization. However, there were no significant differences in the normalized amplitude of simEPSPs or half widths measured at depolarized 

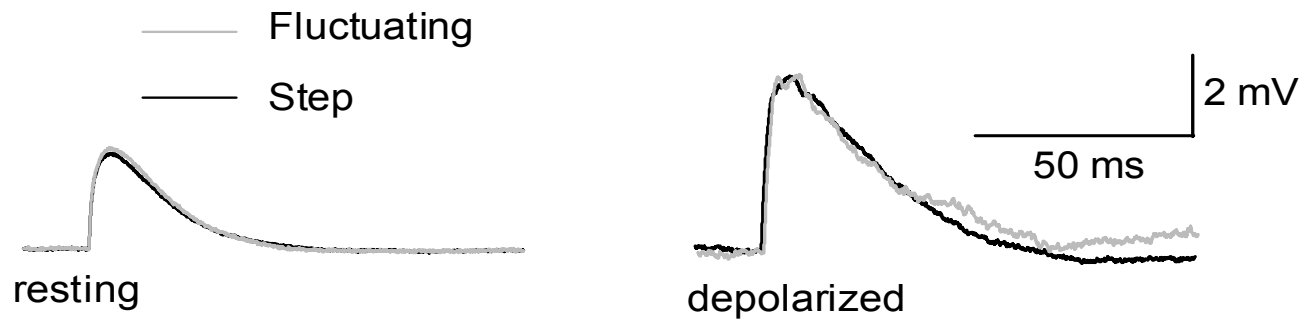

\section{B1}

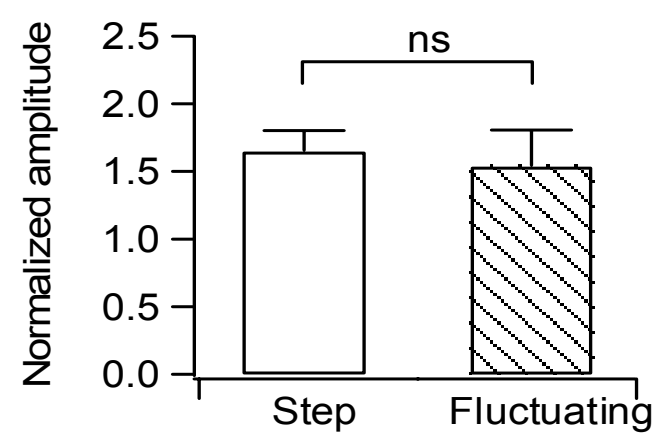

B2
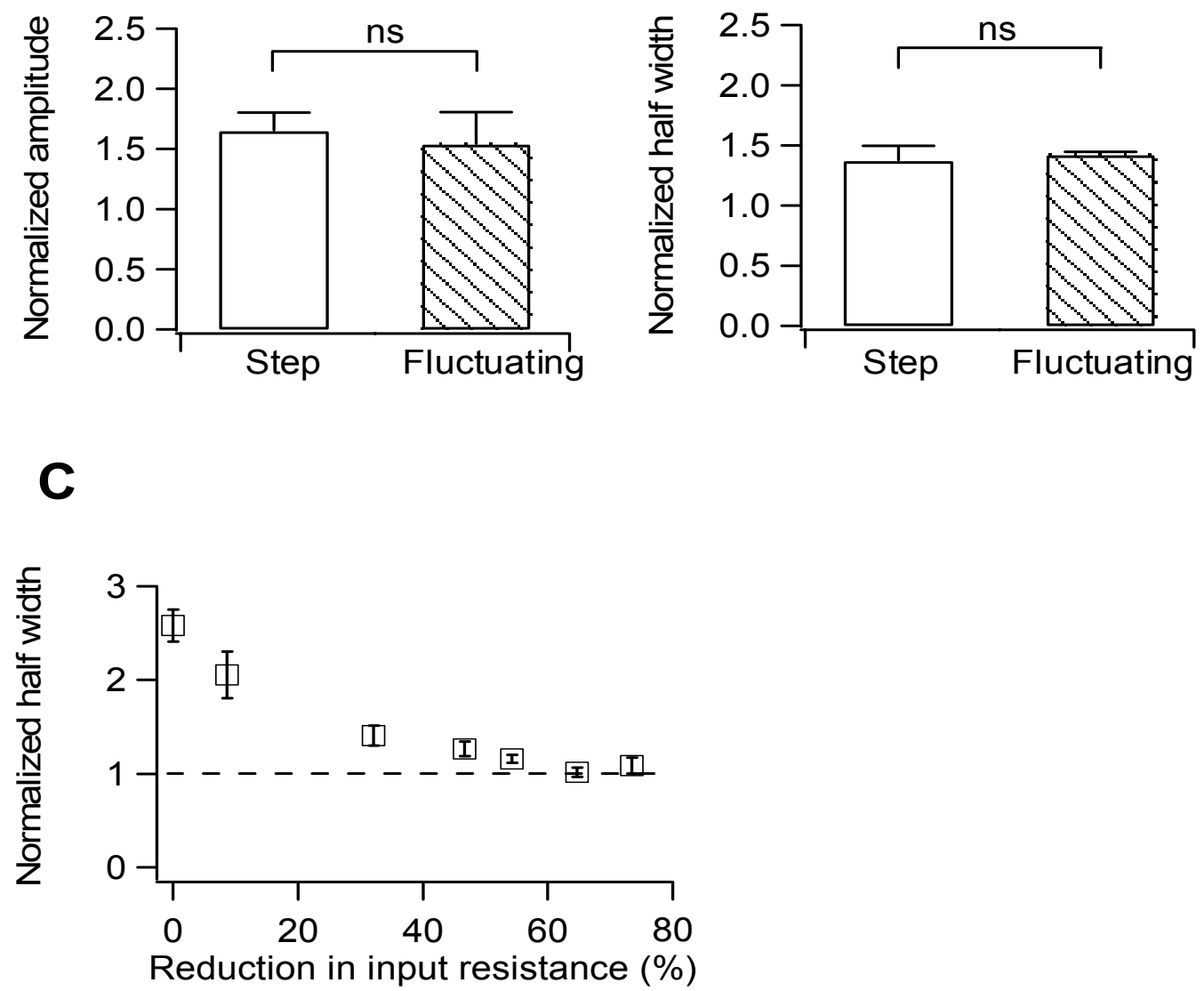

Figure 10. Effect of tonic and fluctuating conductance on EPSP half width. (A) A simEPSC was superimposed either on a conductance step or on a fluctuating conductance wave of the same averages. (B1) Normalized amplitude of EPSP compared to that of at resting potential. (B2) Normalized half width of EPSP compared to that obtained at resting potential. Parameters of EPSP were not significantly different under these conditions ( $\mathrm{p}>0.1, \mathrm{~N}=6$; data from rats (3) and mice (3)). (C) Normalized EPSP half width vs. reduction in the input resistance. SimEPSC was applied together with different levels of tonic conductance and normalized half width of EPSP (depolarized/resting) was measured ( $\mathrm{N}=5$ cells; data from rats (3) and mice (2)). 
membrane potentials and at resting potential under the two conditions. $(1.4 \pm 0.3$-fold with step conductance vs. $1.4 \pm 0.3$-fold with fluctuating conductance for the amplitude (Figure 10A and $\mathrm{B} ; \mathrm{p}=0.329, \mathrm{~N}=6)$ and $1.6 \pm 0.2$-fold with step conductance vs. $1.5 \pm 0.3$-fold with fluctuating conductance for the half width $(\mathrm{p}=0.233, \mathrm{~N}=6)$.

Moreover to ensure that the results above are not related to the amount of conductance used for comparison (20 nS of excitatory and $10 \mathrm{nS}$ of inhibitory conductances) or the specific samples of fluctuating excitatory and inhibitory conductance traces used for stimulation during the experiment. The properties of EPSPs (the sample contained both simEPSPs and eEPSPs) in $(\mathrm{N}=7)$ cells were depolarized with step and fluctuating conductance in interleaving traces were compared. Different fluctuating conductance waves were used for stimulation for each trace when stimulated with fluctuating conductance. The average conductance injected as fluctuating and step conductance was the same in the cell, but the level varied from one cell to another. These included $13 \mathrm{nS}$ excitatory and $4 \mathrm{nS}$ inhibitory or $20 \mathrm{nS}$ excitatory and $10 \mathrm{nS}$ inhibitory or $30 \mathrm{nS}$ excitatory conductance paired with $15 \mathrm{nS}$ inhibitory conductances. Similarly to the previous experiment, there were no significant differences detected between the amplitude, halfwidth and rise-times of EPSP under these two conditions ( $p=0.186$ for the half width, $p=0.537$ for the amplitude and $\mathrm{p}=0.149$ for rise time, $\mathrm{N}=7$ cells; pair-wise comparison with $t$-test).

We also studied how the level of conductance interacts with the properties of simEPSPs. Increasing the level of conductance reduces the half width (Figure 10C), amplitude and risetimes (Table 2) of simEPSPs. Concerning the half-width of the EPSP about 35\% reduction in the input resistance was sufficient to reduce the depolarization-induced EPSP prolongation, and additional conductance could even entirely prevent it (Figure 10C). These results indicate that the level of the background conductance is the main factor in the diminishing of the EPSP half 
Table 2. Effect of conductance on EPSP amplitude and EPSP half-width. E marks excitatory, I marks inhibitory conductances injected. Parallel measurements are from $\mathrm{N}=5$ cells.

\begin{tabular}{|c|c|c|c|c|c|c|c|c|}
\hline & $\mathrm{E}$ & 1 & $\mathrm{E}$ & 1 & $\mathrm{E}$ & 1 & $\mathrm{E}$ & 1 \\
\hline Conductance (nS) & 0 & 0 & 2 & 1 & 10 & 5 & 20 & 10 \\
\hline Reduction in input resistance (\%) & \multicolumn{2}{|c|}{0.0} & \multicolumn{2}{|c|}{10.2} & \multicolumn{2}{|c|}{35.7} & \multicolumn{2}{|c|}{52.9} \\
\hline EPSP amplitude $(\mathrm{mV}) \begin{array}{l}\text { Resting } \\
\text { Depolarized }\end{array}$ & \multicolumn{2}{|c|}{$\begin{array}{l}4.6 \pm 0.3 \\
8.0+0.4\end{array}$} & \multicolumn{2}{|c|}{$4.1 \pm 0.3$} & \multicolumn{2}{|c|}{$3.2 \pm 0.2$} & \multicolumn{2}{|c|}{$\begin{array}{l}2.5 \pm 0.2 \\
4.5 \pm 0.7\end{array}$} \\
\hline \multirow[t]{2}{*}{$\begin{array}{ll}\text { EPSP rise-time (ms) } & \begin{array}{l}\text { Resting } \\
\text { Depolarized }\end{array}\end{array}$} & \multicolumn{2}{|c|}{$\begin{array}{l}3.5 \pm 0.1 \\
6.2 \pm 0.6\end{array}$} & \multicolumn{2}{|c|}{$3.1 \pm 0.6$} & $\begin{array}{l}2.4 \\
3.7\end{array}$ & & \multicolumn{2}{|c|}{$\begin{array}{l}2.3 \pm 0.2 \\
2.7 \pm 0.5\end{array}$} \\
\hline & $\mathrm{E}$ & 1 & $E$ & 1 & $E$ & 1 & & \\
\hline Conductance (nS) & 30 & 15 & 40 & 20 & 50 & 25 & & \\
\hline Reduction in input resistance (\%) & \multicolumn{2}{|c|}{59.0} & \multicolumn{2}{|c|}{65.8} & \multicolumn{2}{|c|}{73.0} & & \\
\hline EPSP amplitude $(\mathrm{mV}) \begin{array}{l}\text { Resting } \\
\text { Depolarized }\end{array}$ & \multicolumn{2}{|c|}{$\begin{array}{l}2.0 \pm 0.3 \\
4.2 \pm 0.3\end{array}$} & \multicolumn{2}{|c|}{$\begin{array}{l}1.9 \pm 0.2 \\
3.4 \pm 0.5\end{array}$} & \multicolumn{2}{|c|}{$\begin{array}{l}1.5 \pm 0.2 \\
3.1 \pm 0.4\end{array}$} & & \\
\hline $\begin{array}{ll}\text { EPSP rise-time (ms) } & \text { Resting } \\
\text { Depolarized }\end{array}$ & \multicolumn{2}{|c|}{$2.0 \pm 0.1$} & $1.9 \pm 0.1$ & & \multicolumn{2}{|c|}{$1.8 \pm 0.0$} & & \\
\hline
\end{tabular}


width prolongation, rather than the membrane potential fluctuation associated with ongoing activity.

We also concluded that the depolarization-induced amplitude increase is present in the layer $\mathrm{V}$ pyramidal cells independently from the amount of conductance injected.

\section{Mechanism of prolongation}

It has been shown that depolarization-induced EPSP prolongation is related to axosomatic sodium channel activation, and the activation of persistent sodium currents (Stuart and Sakmann, 1995, Fricker and Miles, 2000, Andreasen and Lambert, 1999). To test the involvement of the voltage-dependent sodium channels in the simEPSP prolongation and amplitude increase, we measured simEPSP parameters at resting and at membrane potentials close to firing threshold, with and without the presence of TTX. In these experiments tonic conductance was used and the levels of conductance were set at $30 \mathrm{nS}$ for the excitatory and at $10 \mathrm{nS}$ for inhibitory conductances. This amount of conductance almost completely prevented the simEPSP prolongation at depolarized membrane potentials (the normalized EPSP half width was close to 1), but it did not change the normalized amplitude (Figure 10C and Table 2).

Application of TTX at a concentration of $1 \mu \mathrm{M}$ prevented both the increase of simEPSP amplitude and the prolongation of simEPSP half width when the cell was depolarized to membrane potentials close to firing threshold by either a step current or the tonic conductance (Figure 11). The parameters of resulted EPSP were not different from that of measured at resting potential under control conditions (Figure 11). At resting potential the simEPSP amplitude was $4.5 \pm 0.2 \mathrm{mV}$ and $4.2 \pm 0.2 \mathrm{mV}$ without TTX and when TTX was present and there was no 


\section{Current}
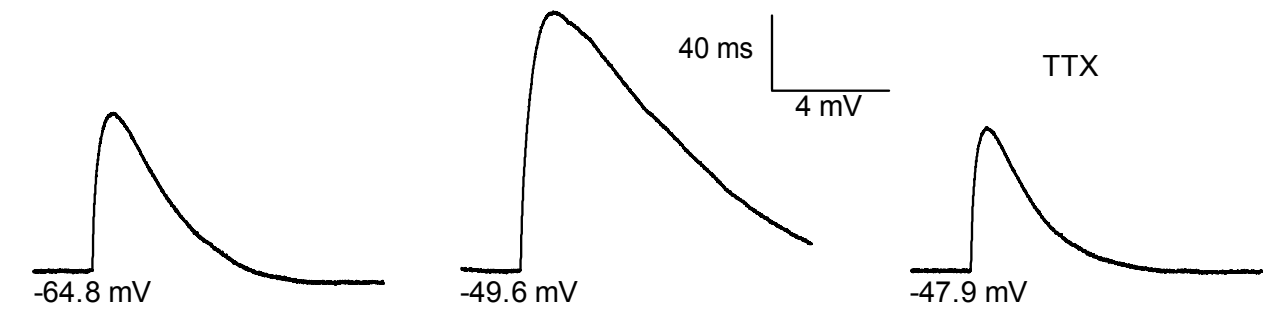

TTX

\section{Conductance}
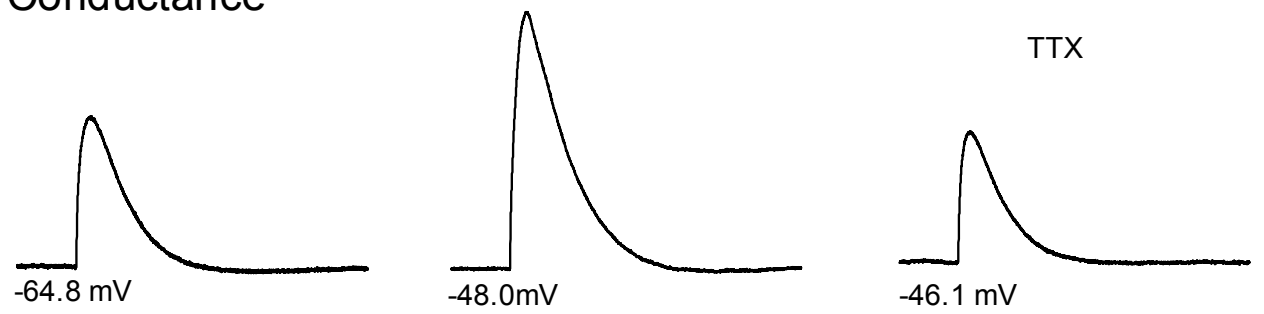

Figure 11. Prolongation of simEPSP is related to the activation of sodium channels.

Parameters of simEPSP were measured at resting potential, and at membrane potentials close to the firing threshold. The cell was depolarized by either a current step or static conductance. SimEPSP was scaled to generate EPSPs of similar size at resting potential under both conditions. Application of TTX at a concentration of $10 \mathrm{mM}$ prevented both prolongation and amplitude increase of EPSPs due to depolarization. 
conductance injected $(\mathrm{p}=0.285, \mathrm{~N}=4$ cells). Under similar conditions, the EPSP half with was $22.9 \pm 0.8 \mathrm{~ms}$ and $22.5 \pm 0.7 \mathrm{~ms}$ without TTX and TTX, respectively $(\mathrm{p}=0.264, \mathrm{~N}=4)$.

When conductance was present at resting potential, EPSP amplitude was $3.9 \pm 0.1 \mathrm{mV}$ and $3.7 \pm 0.2$ without and with TTX applied, respectively $(\mathrm{p}=0.0 .219, \mathrm{~N}=4)$. The EPSP half widths were $14.9 \pm 0.4$ without TTX and $14.7 \pm 0.4$ with TTX $(p=0.078, N=4)$ in the presence of the given conductance. We also measured what happens with the EPSP when the cell was depolarized. The normalized amplitudes were $1.6 \pm 0.1$ and $1.0 \pm 0.04$ without TTX and with TTX, respectively, when the cell was depolarized with a step current $(\mathrm{p}=0.004, \mathrm{~N}=4)$. The normalized half widths of EPSPs were $2.4 \pm 0.3$ and $1.0 \pm 0.03$ without and with TTX, respectively, for the same conditions $(\mathrm{p}=0.021, \mathrm{~N}=4)$. When the cell was depolarized with conductance, the normalized amplitudes were $1.4 \pm 0.1$ and $1.0 \pm 0.07$, without and with conductance, respectively $(\mathrm{p}=0.001, \mathrm{~N}=4)$. Depolarization with conductance resulted in normalized EPSP half widths of $1.0 \pm 0.1$ and $1.0 \pm 0.1(\mathrm{p}=0.246, \mathrm{~N}=4)$.

These results show that the increase in the amplitude and in half width of simEPSP due to depolarization is related to the activation of voltage-dependent sodium channels, because blocking these channels removes the effect.

\section{Background conductance improve spike timing precision}

We hypothesized that the presence of depolarization-dependent prolongation of EPSPs at membrane potentials close to firing threshold prevents pyramidal cells from responding to EPSPs with precise firing (Fricker and Miles, 2000, Maccaferri and Dingledine, 2002). If this 
hypothesis is true, then a condition, which reduces EPSP prolongation close the firing threshold, may improve the precision of spike timing.

Cells were depolarized with a current step a conductance step (Figure 12A and B) in interleaved traces, and the average latency of spikes generated in response to the simEPSP was measured (Figure 12A2 and B2). The level of depolarization (both with current and conductance) and the applied simEPSC together produced spikes with a probability between $0.3-0.8$ after the onset of simEPSP in every experiment. To quantify the distribution of spike timing under the two conditions, we defined a spike time window. Cumulative probabilities of firing (Figure 12C1) were calculated from the latencies of spikes from the onset of the EPSP and the spike time window was measured between the points corresponding to 0.1 and 0.9 probabilities in the cumulative histogram of the probability of firing (Figure 12C). In all experiments, pyramidal cells showed a shorter spike time window when depolarized with background conductance (Figure $12 \mathrm{C} 2$ and D). Also, when the cell was depolarized with conductance, the EPSP half width was shorter, similarly to Figure 8 . and 9. (data not shown). The data together with the data shown previously supports the hypothesis that increased precision correlates with a reduction in the half width of the simEPSP at more depolarized membrane potential values. Spike windows were $44.9 \pm 9.6 \mathrm{~ms}$ and $14.9 \pm 3.6 \mathrm{~ms}$ for the current and conductance, respectively $(\mathrm{p}=0.004$, $\mathrm{N}=8$ cells). In a separate set of experiments $(\mathrm{N}=9$ cells), which was stimulated in interleaving traces with fluctuating conductance and step current, the effect of fluctuating conductance was evaluated on the spike time window and was compared to the spike time window generated from traces depolarized by a current step and tonic conductance step. The average spike time window was $9.2 \pm 3.6 \mathrm{~ms}$ in the presence of fluctuating background conductance, which was not 
Figure 12. Effect of background conductance on the precision of spike timing. (A) Cells were depolarized close to firing threshold by a current step and a simEPSP was superimposed on the background activity. (B) Same as (A), except cells were depolarized with a step conductance. (A2) and (B2) Latency of spikes due to the simEPSP was measured (histogram bin width is 1 $\mathrm{ms}$ ). (C) Cumulative probabilities from the latency of spike histograms: rise times of cumulative histograms measured as time-span between 0.1 and 0.9 probabilities $(\mathrm{C} 1)$ is defined as the spike time window. (C2) Spike time window is always shorter, when the cell is stimulated with conductance compared to when stimulated with current. Experiment pairs on the same cells are marked with the same symbols. (D) Length of spike time windows when stimulated with step current (empty bar), tonic conductance (bar with stripes) and fluctuating conductance (filled bar). (E) Reduction in spike time window vs. the amount of reduction in the input resistance. Sample traces on (A), latency histograms on (B1) and cumulative probabilities on (C1) are from the same cell. 
Current

A1

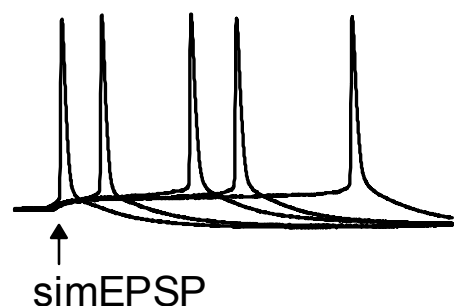

A2

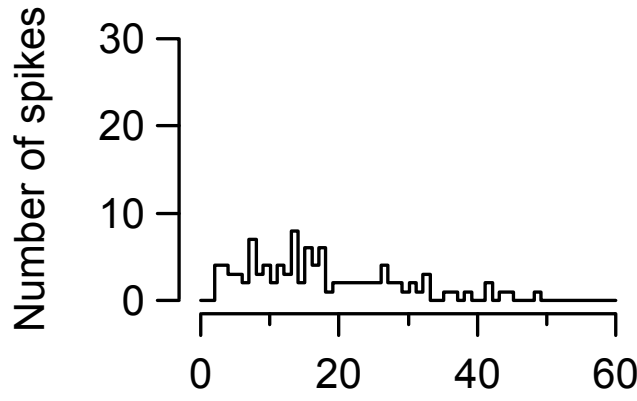

Latency to first spike (ms)

C1

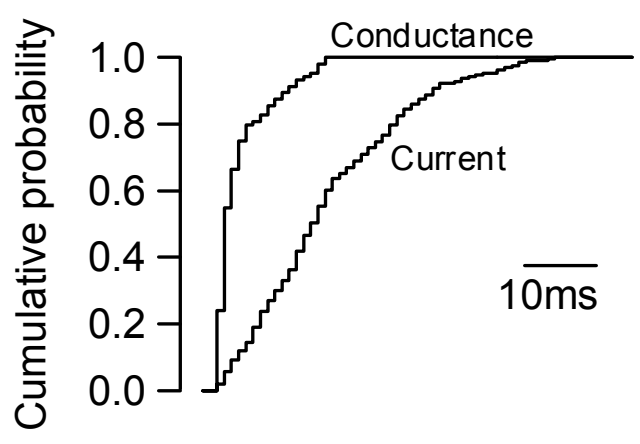

D

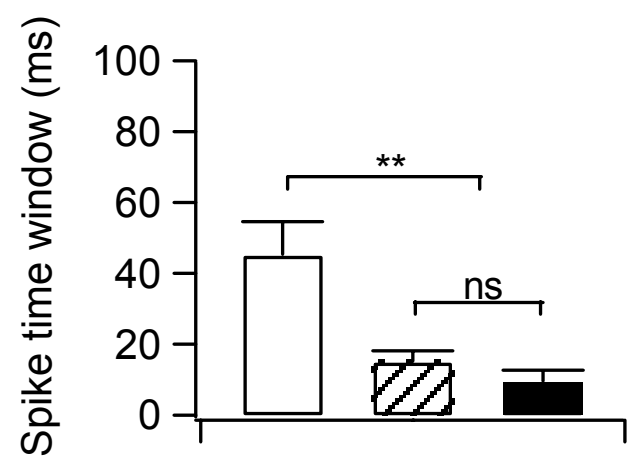

Conductance

B1

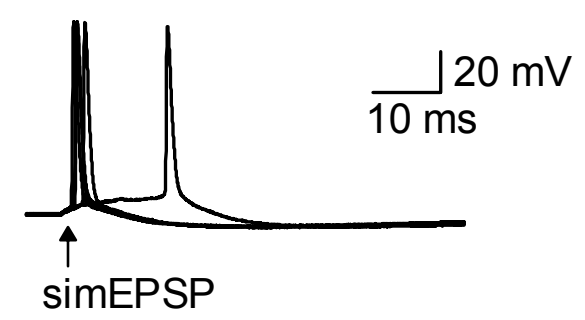

B2

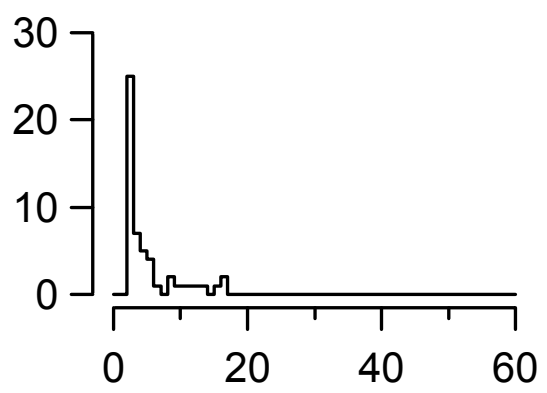

Latency to first spike (ms)

C2
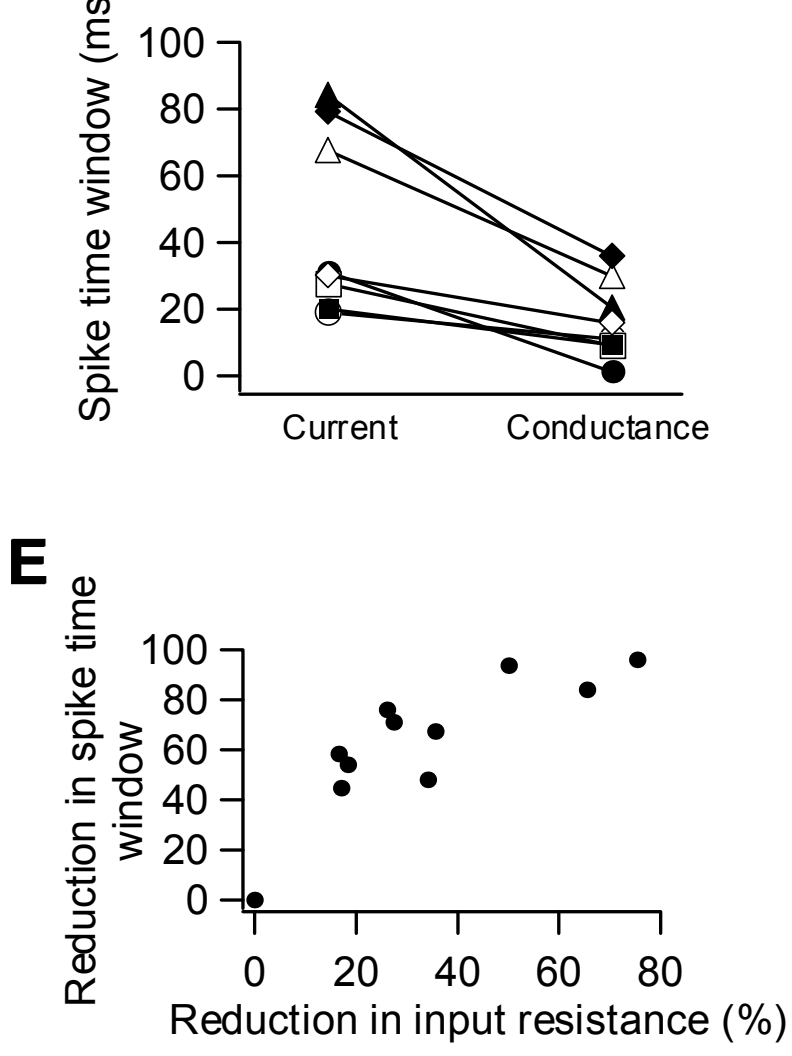
significantly different from that measured in the presence of the step conductance, (independent t-test, $\mathrm{p}=0.273, \mathrm{~N}=9$ ).

We also tested the relationship between the reduction of the spike time window and the reduction of input resistance of the cell by the injection of tonic conductance in the experiment pairs on Figure $12 \mathrm{C} 2$. When the values of reduction, defined as Spike time window CURRENT STEP- $^{-}$

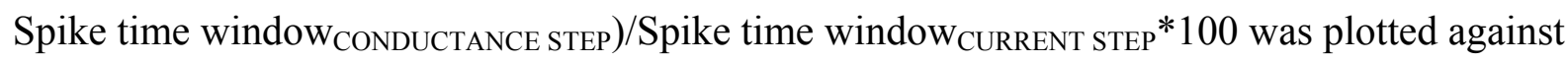
the reduction in the input resistance the points showed a step decrease with the increasing input resistance. When fitted with a single exponential curve the tau value was about $23 \%$, meaning that a $23 \%$ reduction in the input resistance caused a $66 \%$ reduction in the spike time window.

Thus, these data indicate, that background conductance improves temporal precision of EPSP-spike coupling in pyramidal cells by reducing the depolarization-induced prolongation of EPSP time course.

\section{Relationship between PSTH and EPSP properties}

Background synaptic activity might increase spike-timing precision in cells with no ongoing spiking activity (Figure 12). However cortical cells fire continuously due to their constant synaptic drive reflecting the activity of the presynaptic neurons in the network. The reported discharge frequency in awake animals has a range of $5-50 \mathrm{~Hz}$, as reported from experiments made with single unit (Mountcastle et al., 1969, Hubel, 1959) and intracellular (Steriade et al., 2001) recordings. In anesthetized animals the discharge frequency is somewhat lower, but it is never ceased (see references above). A recent study, however, using in vivo whole 
cell patch-clamp recording, reported much lower values for both in awake and anaesthetized animals (Margrie et al., 2002).

It has been suggested based on the results on spinal cord motoneurons with ongoing activity that a single EPSP transiently increases the probability of discharge, and the time-course of this increased firing probability is shorter than the time-course of the EPSP itself (Cope et al., 1987, Fetz and Gustafsson, 1983, Kirkwood and Sears, 1982, Poliakov et al., 1997). The probability of firing related to the stimulus can be plotted in the peristimulus time histogram (PSTH; see Experimental Methods). Therefore the relationship between PSTH and EPSP properties of cortical pyramidal cells was experimentally determined similar to Fetz et al. (1991) and Matsumura et al. (1996).

We injected background activity of fluctuating conductance that created a firing frequency of 15-30 Hz (background firing frequency), and an eEPSP was superimposed on the top of it. EPSP amplitude and half width were measured at resting potential with no conductance in the background. The amount of injected conductance was the same in these experiments. Peak synaptic gain was defined as an increase in the probability of spike generation due to an EPSP (see Experimental Methods). In order to calculate peak synaptic gain, we compiled two PSTHs: from traces stimulated with background activity only (Figure 13A1 upper panel) and from traces stimulated with an eEPSP superimposed on the fluctuating activity (Figure 13A1, lower panel). The peak synaptic gain and the time window of the peak synaptic gain was measured using the cumulative sum from the two PSTHs: one is generated from traces with background stimulation only and the other is generated from traces where background stimulation was applied together with an EPSP (See Experimental Methods about the generation of cumulative sums.). The rising phase on the cumulative sum corresponded to the peak synaptic gain. The maximum of the 
A1
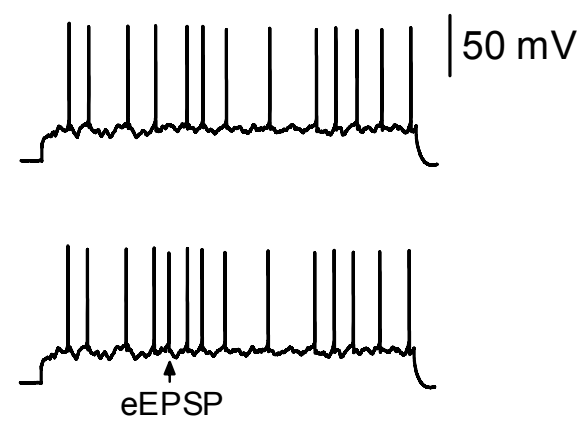

$12 \mathrm{mV}$

\section{A2}

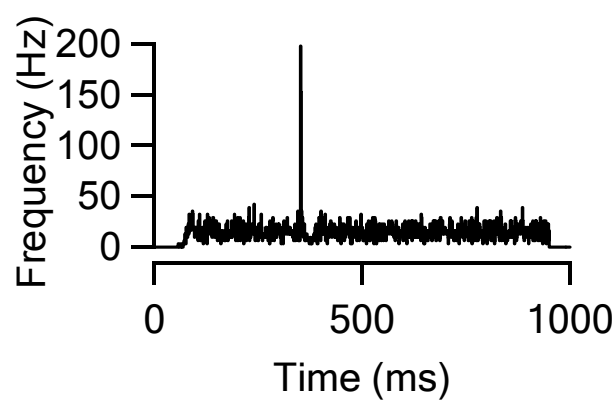

A3

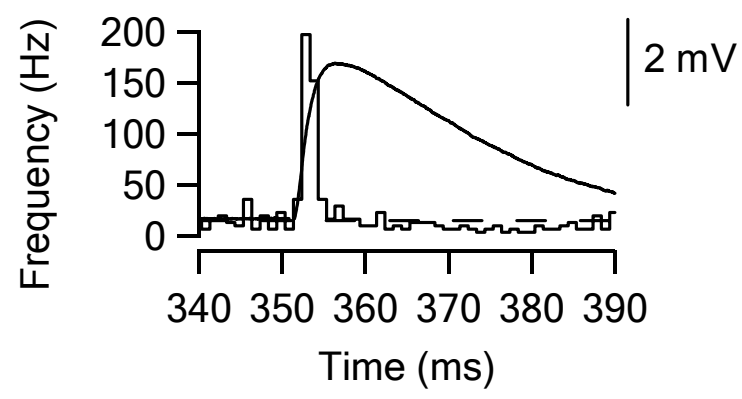

B

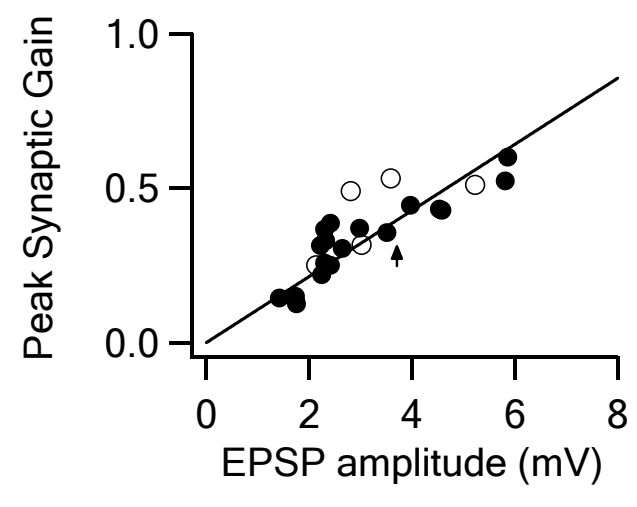

Figure 13. Effect of eEPSP amplitude on peak synaptic gain. (A) Peristimulus time histograms (PSTH) were compiled from $\mathrm{N}=307$ traces stimulated with an eEPSPs (A1) middle panel. Background stimulation without applying an eEPSP is shown on the upper panel and the timing of eEPSP is shown on (A1) lower panel. (A2) Peristimulus time histogram from traces with eEPSP stimulation. (A3) PSTH and eEPSP at high resolution. Dashed line symbolizes average baseline activity. (PSTH with no eEPSP stimulation is not shown.) (B) Peak synaptic gain versus EPSP (eEPSP: filled circle, $\mathrm{N}=19$ cells; simEPSP: empty circle, $\mathrm{N}=5$ cells). 
cumulative sum gave the number of the peak synaptic gain, because the baseline values were zero after the subtraction of two PSTHs. The time window of the peak synaptic gain was defined as a period between $10 \%$ and $90 \%$ of the maximum value of the cumulative sum.

There were two characteristics of the spike timing observed from the comparison the two kinds of PSTH (Figure 13A2 and A3):

(1) EPSPs evoked spikes only during the rise time of EPSPs, which is in agreement with the previous findings (Cope et al., 1987, Fetz and Gustafsson, 1983, Kirkwood and Sears, 1982, Poliakov et al., 1997).

(2) Spiking probability was reduced after the PSTH peak, and we observed a "loss" in the number of spikes relative to the control traces when compared to the value of baseline activity (dashed line, Figure 13A3). Since the reduced activity differ only a small value from the baseline activity, we used cumulative histograms to study its properties in following part of the study.

The peak synaptic gain was plotted versus EPSP amplitude, both for eEPSP and simEPSP (Figure 13B). The EPSP amplitude and peak synaptic gain had a linear relationship with about $10 \%$ increase in the probability of spiking with every $1 \mathrm{mV}$ increase in the amplitude of EPSP. These results were similar to those reported in cat spinal cord motoneurons (Gustafsson and McCrea, 1984, Cope et al., 1987), and cortical neurons measured in vitro (see reference in Fetz et al. (Fetz et al., 1991), but lower than in monkey cortex measured in vivo (Matsumura et al., 1996).

Evoked EPSPs had a rise time range of 1.6-6.6 ms, and resulted in peak synaptic gain with time windows of 0.8-7.3 ms. Average time window of the peak synaptic gain was $1.4 \pm 5.8$ ms $(\mathrm{N}=19)$. The large variability of the average time window may be attributed to the variability in eEPSP rise times. The rise time of eEPSP and the time window of the peak synaptic gain was 
correlated with a $\mathrm{r}=0.67,(\mathrm{p}<0.05, \mathrm{~N}=19)$. These results show that EPSP amplitude and rise time are critical in determining the peak synaptic gain.

Neurons are characterized by a refractory period after a spike, which is related to the inactivation of sodium channels and activation of potassium channels (Hodgkin and Huxley, 1952). It is not possible to generate a spike during the absolute refractory period, which follows the spike immediately. During the relative refractory period only a stronger stimulus can evoke an action potential. If we use a stimulus just strong enough to generate a spike with a probability of 1 , then a stimulus of the same size would trigger an action potential with less likelihood if it arrives during the relative refractory period. Using the definition above, auto renewal functions of firing were calculated (See Experimental methods). The auto renewal function was fitted with a sigmoid curve (See description of the fitting in the Experimental Method). The refractory period was characterized as the period necessary to reach half of the average firing rate after spikes (xhalf). The range of values of the refractory period was $18-45 \mathrm{~ms}$ with an average of $26.7 \pm 7.8 \mathrm{~ms}$ (Figure 6; $\mathrm{N}=11$ ).

Next the possible perturbation of action potential patterns arising from an EPSPgenerated spike was examined. Suppose that a spike train was generated by a given background stimulation. A new spike due to an EPSP that was not part of the original background stimulation may disturb the probability of occurrence of the spikes following the perturbation. The term "loss of spikes" was defined to measure the probability that the spike count of a spike train does not increase after a transient increase due to the EPSP (peak synaptic gain). It is calculated as the average value of the longer, stabilized part of the cumulative sum after the initial peak due to the EPSP, normalized to the peak synaptic gain. The "new" spike generated by the EPSP may cause a realignment of the timing of the subsequent spikes resulting in a disappearance of a subsequent 
spike generated by the background activity. Or, the spike generated due to the EPSP causes a period of limited excitability during its refractory period.

An important condition for this analysis is that spike generation mechanism can be precise and reliable in pyramidal cells (Mainen and Sejnowski, 1995) in response to stimulations that generate high frequency fluctuations in the membrane potential (Mainen and Sejnowski, 1995, Nowak et al., 1997). For that reason, the reliability of spike trains was measured as was described by (Mainen and Sejnowski, 1995), with slight modifications. Briefly, fluctuating background conductance was used to generated a discharge frequency of 4-25 Hz, and a histogram was calculated from the timing of spikes from 50 traces with a bin width of $1 \mathrm{~ms}$. After smoothing (9 step boxcar filter, see Experimental Methods), the reliability was measured as the probability of getting the spikes in the peaks as opposed to outside the peaks of PSTH. Peaks were defined as an increased probability of discharge above a threshold, which was 3-fold the average background frequency. The two immediate neighboring bins of from each side of the peak that contained a probability of discharge below the threshold was also included. The reliability ranged from 0.68 to 0.98 with an average of $0.85 \pm 0.04(\mathrm{~N}=10)$. In comparison when reliability was measured with step current stimulation, the average reliability was $0.35 \pm 0.05$ with a range of $0.18-0.52(\mathrm{~N}=6)$. The difference between the two groups was significantly different ( $t$-test for independent samples, $\mathrm{p}<0.001)$.

We concluded from these data that fluctuating conductance generates spike trains occurring with high reliability.

The refractory period depends on the properties of the cell, it limits the frequency of firing, and reduces the variability of spike timing, imposing a more regular spiking activity (Gray, 1967, Gaumond et al., 1982). Refractory period also offers a possible mechanism for the 
regulation of the net synaptic gain, because in a cell discharging with high frequency the chance that an additional spike due to the EPSP arrives during the refractory period of a spike is higher, which would decrease the probability of spike generation. An additional synaptic input to a cell with ongoing spiking activity shortens the interspike interval transiently. If this input is strong enough to generate a spike, then a peak will occur on the PSTH. Then a spike on the stimulated train will occur at the time of the peak synaptic gain and at the same time a spike which otherwise would occur due to background synaptic activity disappear, as it seen on the control trace. Such a stimulus cannot add to the net gain, but adds to peak synaptic gain. To analyze the relationship between firing frequency and the "loss of spikes", fluctuating conductance waves were used to evoke spiking with a frequency range of 1-25 Hz (Figure 14A and B) and simEPSP parameters kept constant in every experiment (amplitude: $400 \mathrm{pA}$, rise time constant: $0.1 \mathrm{~ms}$, decay time constant: $5 \mathrm{~ms}$ ). To generate the desired frequencies, a constant hyperpolarizing or depolarizing current step was added to the background conductance. Cumulative histograms were calculated (see in Experimental Methods) to measure the distribution of simEPSP-related spikes in time (Figure 14A3 and B3). The dependence of "loss of spikes" and the frequency was determined. At lower frequencies the "loss of spikes" was close to 0 , and the relationship between the "loss of spikes" and background firing frequency was steep, and close to 1 at higher frequencies. At the same time, background frequency showed no relationship to the peak synaptic gain (Figure 14D; r=0.03).

Our results show that two major factors contribute to the efficacy of EPSP-spike coupling, synaptic gain and synaptic background activity. The EPSP amplitude determines the peak synaptic gain, while its time course is related to the time window of the peak synaptic gain; 
Figure 14. Relationship between firing frequency and spike loss. (A) and (B) Background activity of fluctuating conductance created a discharge frequency of 1-24 Hz. A simEPSC with amplitude of $400 \mathrm{pA}$ was added and PSTHs were compiled from traces with simEPSPs (A1 and B1). (A1) PSTH from an experiment with a low background firing frequency $(2.1 \mathrm{~Hz}, \mathrm{PSTH}$ made from $\mathrm{N}=381$ traces). (B1) PSTH from an experiment with a higher background firing frequency (14.6 Hz; PSTH is from $\mathrm{N}=400$ traces). (A2) and (B2) shows PSTHs on (A1) and (A2) with higher resolution, respectively. (A3) and (B3) Cumulative sum of PSTHs from the experiments to follow the time course of probability changes in spike generation due to simEPSP. (C) Relationship between the loss of spikes and background frequency ( $\mathrm{N}=29$ cells). (D) Peak synaptic gain and background frequency from the cell on $(\mathrm{C}),(\mathrm{r}=0.03)$. 
A1 Background frequency: B1 $2.1 \mathrm{~Hz}$

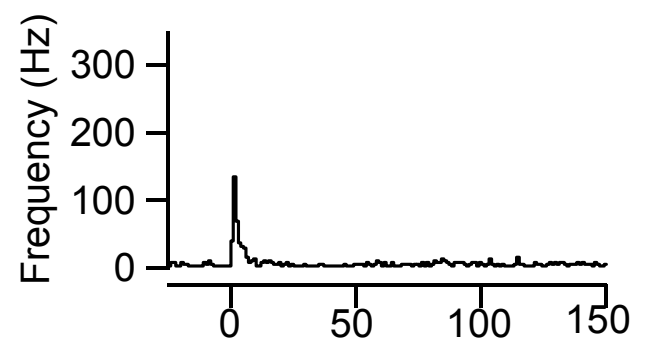

A2

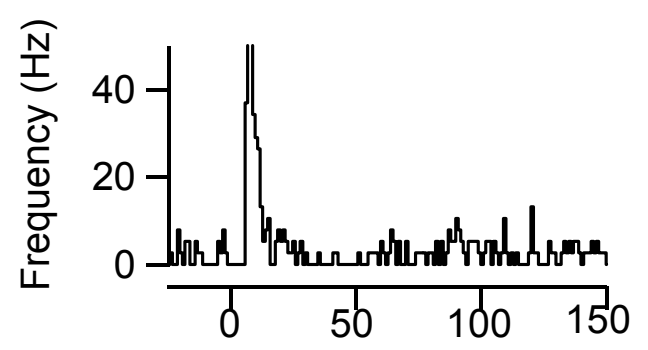

A3

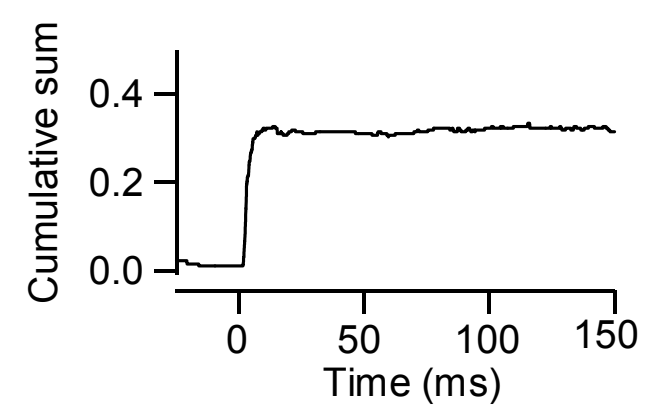

C

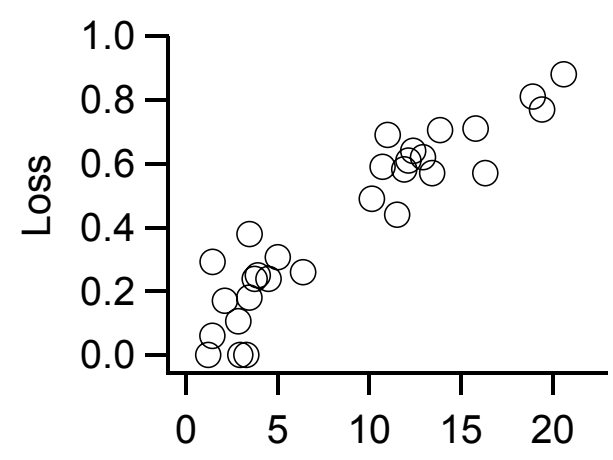

Background frequency $(\mathrm{Hz})$
Background frequency: 14.6 Hz

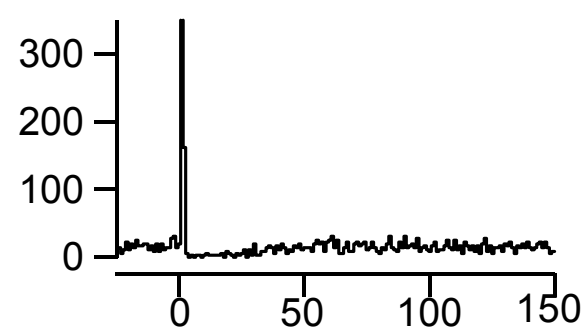

B2

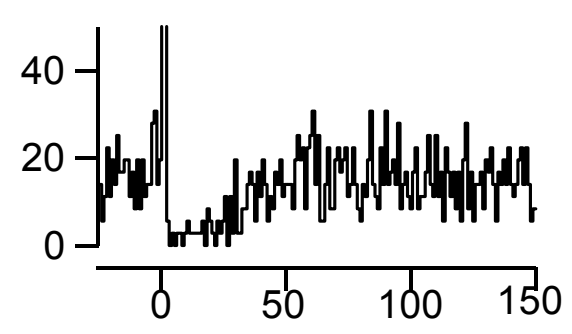

B3

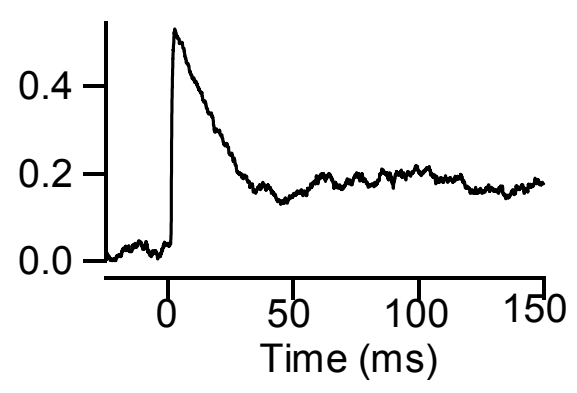

D

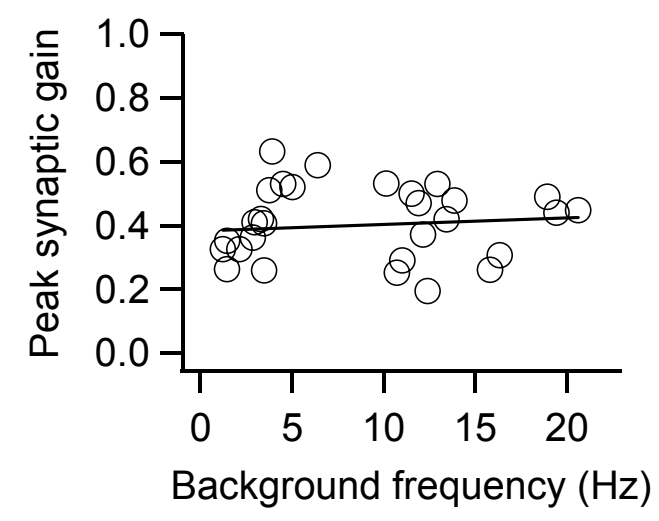


and the synaptic background activity that generates ongoing discharge, which in turn reduces the probability of spiking after the peak synaptic gain.

\section{The role of fluctuation in the background activity}

Background stimulation with low variability produces a regular discharge pattern, in which the timing of spikes depends mainly upon the timing of the previous spikes (Figure 15A1 and A2). We characterized the regularity of spiking by calculating the coefficient of variation (CV) of the interspike intervals. We defined a spike train as regular, if the coefficient of variation was under 0.15 . Spike trains evoked by step current were always regular, with an average $\mathrm{CV}$ of $0.1 \pm 0.01$

How the regularity in the interspike interval related to the "loss of spikes" and consequently, if an irregularity in the spike timing can prevent the "loss of spikes" remained an open question: This was hypothesized that regularity in spiking related to the loss of spikes on the PSTH and any mechanism that introduces irregularity in spike timing may increase the efficacy of EPSP-spike coupling. To address this question, the cells were stimulated in interleaving traces of step current or fluctuating conductance that produced a firing frequency of $2-10 \mathrm{~Hz}$. The protocol was the following in every experiment: first trace was a stimulation with step current (Figure 15A1 and A2, solid lines), which was followed by the same step current with and simEPSP evoked on the top of it (Figure 15A1 and A2 dashed lines). The third trace was stimulated with fluctuating conductance (Figure 15B1 and B2 solid lines) and the forth contained the same fluctuating conductance with a simEPSP evoked on the top of it (Figure 15B1 and B2 dashed lines). During the experiment the same fluctuating conductance was repeated. 


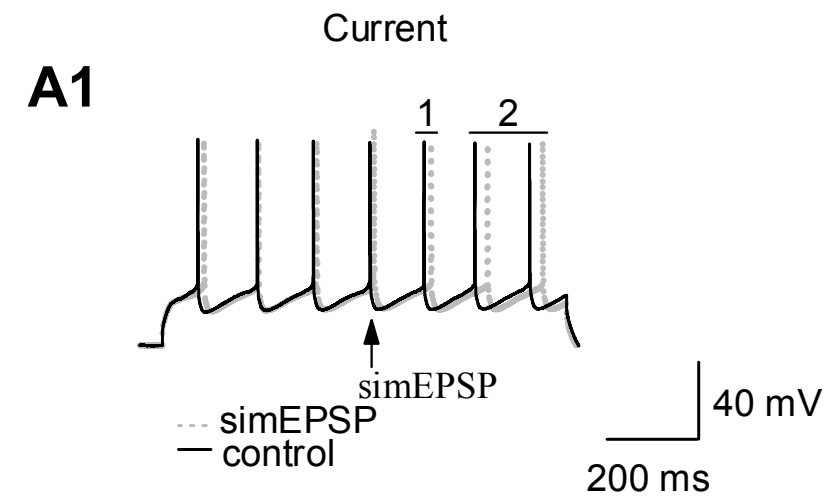

B1

Conductance

A2

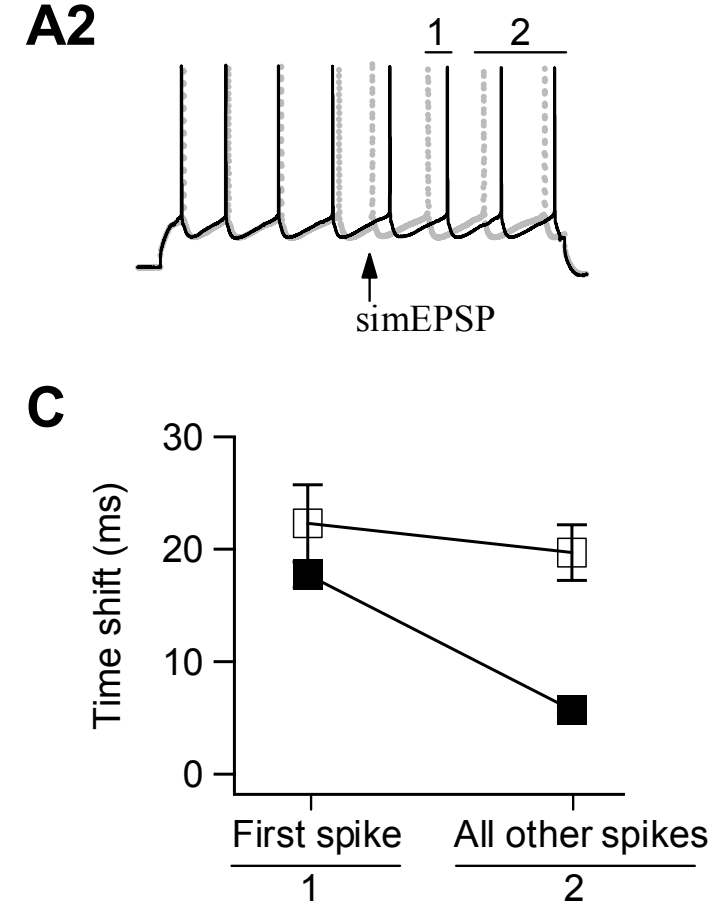

B2
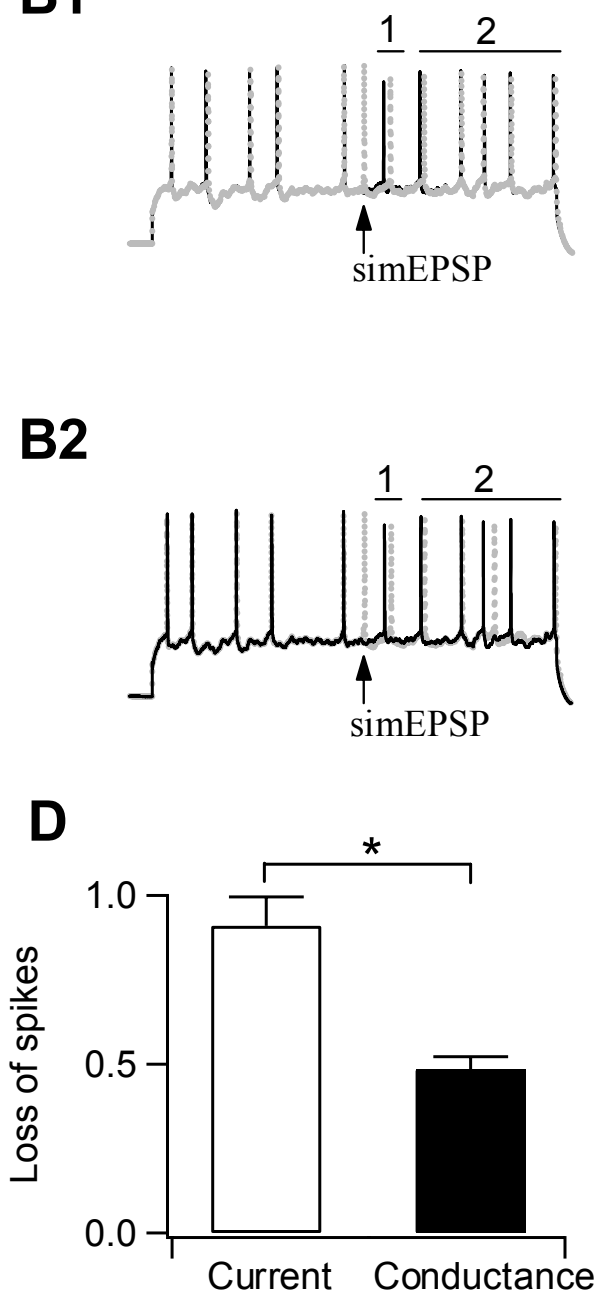

Figure 15. Effect of fluctuating conductance on spike loss. Spiking was evoked either by step current (A) or fluctuating conductance (B) and the effect of a simEPSP related spikes (simEPSPspike) were studied on the cell's firing. Traces on (A) and (B) are single examples. Interleaving traces of background stimulation only (solid) and stimulation with EPSP (gray dashed line) were collected. With current step simEPSP-spike resulted in either no change at all (A1) or in a shift in the timing of all spikes (A2) occurring after the simEPSP-spike. With fluctuation, an extra spike could be generated with no loss (B1) or simEPSP-spike suppressed another spike somewhere later in the spike train. (C) Comparison of the absolute time shift in spike timing related to simEPSP-spike under the conditions in the experiment marked with 1 and 2 on panels (A) and (B). (D) Comparison of loss of spikes under the two conditions $(\mathrm{p}<0.05, \mathrm{~N}=6$ cells). 
In all experiments, when stimulated with step current, the firing frequency was lower or equal to the level of that stimulated with fluctuating conductance. When the EPSP arrived in the middle of an interspike interval, it shortened it and increased the discharge frequency transiently (Reyes and Fetz , 1993). Because the amount of current that evoked spiking did not change in the longer time scale, the cell continued firing with the same frequency after this transient increase.

Consequently, the timing of all the subsequent spikes was also affected (Figure 15C). We should note here, that in certain cases the EPSP prolonged the interspike interval instead of shortening it. In these cases the membrane potential deflection due to the EPSP looked similar to those on Figure 12A1, measured under at below spike threshold depolarization. Nevertheless, a change in the interspike interval length due to the EPSP was preserved in the following interspike intervals.

An extra spike due to the simEPSP, which was not part of the spike train in the control traces, caused a delay in the first following spike (Figure 15B1), which in turn could influence the timing of following spikes (Figure 15A2 and C). However, the amount of delay decreased monotonically towards the end of the spike train (see below a more quantitative analysis). About $50 \%$ of the traces showed spike loss under this experimental condition: a spike was suppressed relative to the control, due to the simEPSP related spike, but not necessarily during the relative refractory period of that spike (Figure 15B2). The probability that a spike in the train disappeared after a single spike was generated due to the EPSP (called the loss of spikes) was $0.9 \pm 0.1$ when stimulated with a step current, and $0.5 \pm 0.1(\mathrm{p}=0.036, \mathrm{~N}=6)$, with fluctuating conductance.

In summary, the presence of fluctuation in the background activity regulated how an EPSP-evoked spike affected spike timing in the spike train. This in turn affects the how spikes are accumulated in the spike train and determines the net synaptic gain. 


\section{Effect of fluctuating background conductance on perturbation of spike timing}

It is assumed that the messages conveyed through the axon are contained in a series of time intervals. This gives rise to two important questions: (1) How long should these time intervals need to be to convey the information reliably, and (2) do these epochs contain independently encoded information, or does the neuron keep a "memory" about the previous messages and use it for coding further messages. In many studies spike timing is considered to be a Poisson process in which the individual spikes are independent from other spikes. However, in a neuron with ongoing discharge, the timing of spikes may be determined not only by the stimulus but also the presence and timing of the previous spike(s). We can imagine a cell in which the timing of a single spike is affected by some perturbation, and in which this perturbation generates a perturbation in the next spike, which in turn creates a perturbation in all the subsequent spikes. It is hypothesized that the interplay of slowly activating and non- or slowly inactivating sodium and potassium conductances that are underlying spontaneous oscillation at membrane potential levels close to firing threshold allow the influence of a spike in a train to extend several hundred milliseconds by causing a "jitter" in the spike timing (Gutfreund et al., 1995).

Another coding related question is how neurons handle noise from EPSPs arriving with stochastic patterns. If the integration time window is short, the inputs participating in spike generation should arrive synchronously. It is hypothesized that a system utilizing temporal coding is more susceptible to the transmission of erroneous input than one that uses rate coding, because the number of inputs for spike generation is more limited with temporal coding. As conductance improves the precision of spike timing, but arrival of a single spike can influence 
the timing of other spikes. It remained to be determined as to how long can a single spike perturb the spike train, and whether conductance has an influence on that process?

We injected fluctuating current or fluctuating conductance in interleaving traces to evoke spike trains in $\mathrm{N}=7$ experiments. The purpose of the analysis was to assess whether the presence of conductance in the stimulation shortens the recovery of spike trains from perturbation compared to stimulation with fluctuating current. In this way the role of conductance could be addressed separately from the role of membrane potential fluctuation (Figure 16A and B). To preserve the kinetic properties of the background stimulation we used the same unitary transients for generation of fluctuating current waves (see Experimental Methods for the description of the procedure). With the alternating stimulation of fluctuating current and fluctuating conductance, the corresponding traces of fluctuating current and conductance generated spike trains with similar frequency of discharge, in a range of 3.9-5 and about $15.6 \mathrm{~Hz}$ in the experiment pairs ( $N=7$ cells). Alternating traces of background stimulation only and with a simEPSP superimposed on the background stimulation were used.

Two kinds of perturbation were tested: (1) an EPSPs did not result in a spike and (2) EPSP that resulted in spikes during the peak synaptic gain. The effect of simEPSPs that did not generate a spike, was examined by measuring how much the timing of spikes subsequent to the simEPSP advanced compared to the control traces. Since simEPSP did not generate a spike in the first case, but a depolarization in the membrane potential compared to traces stimulated with the background conductance only, spikes were hypothesized to advance on the trace with simEPSP when compared with the trace with the background stimulation. This advancement was measured as a difference between the timing of spikes on the traces with simEPSP and the timing of spikes with background stimulation only, then the advancement values were grouped 
Figure 16. Perturbation of spike timing following an action potential generated by an EPSP. (A) The cell was depolarized to near threshold by an injection of a fluctuating current wave. The action potential train in control (dark trace) and when a simEPSP generated an action potential (gray dashed trace; indicated by the arrow). Note the delayed action potential generated by the simEPSP (gray dashed trace) and the perturbation of the following action potential relative to the control action potential train (dark trace). (B) The cell was depolarized with an injection of a fluctuating conductance wave. A simEPSP generated a spike, which a caused relatively small perturbation in the spike timing. (A1) and (B1) show full traces, and (A2) and (B2) are blown-up versions, marked by the horizontal lines on (A1) and (B1). (C) Recovery of spike trains from perturbation in the experiments on (A) and (B), as shown by the cumulative delays in the in spike timing. Arrows mark the perturbation window: a spike, arriving after this point had less than $5 \%$ chance to be affected by the presence of simEPSC related spike. (D) Perturbation window in spike trains generated by fluctuating current and fluctuating conductance $(p<0.05, N=6)$. (E) Comparison of loss of spikes in the presence of fluctuating current and fluctuating conductance on individual experiments $(\mathrm{N}=7)$. Values generated with stimulation of fluctuating current and fluctuating conductance on the same cell are connected with the line. 
A1

Fluctuating current

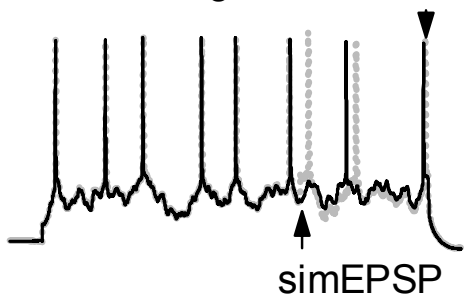

A2

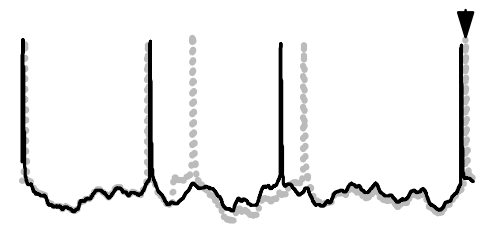

C a Fluctuating current

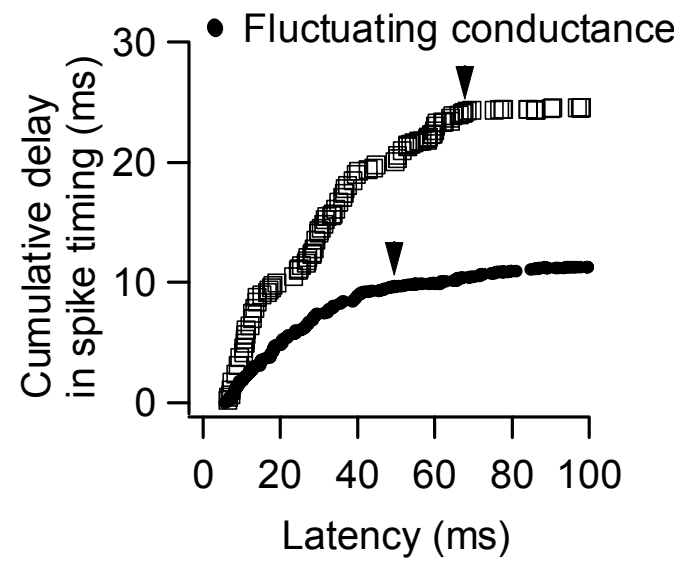

E

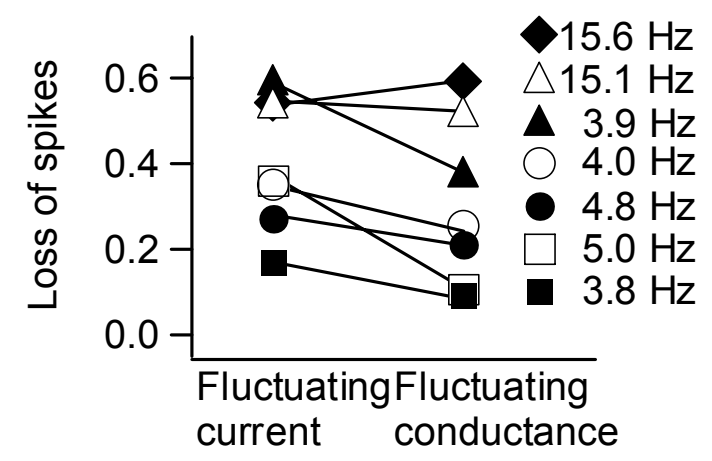

B1

Fluctuating conductance

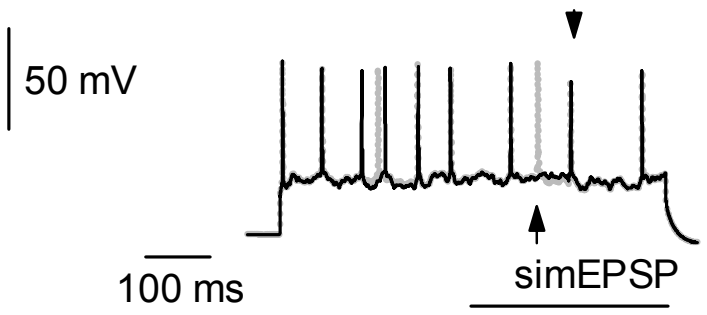

B2

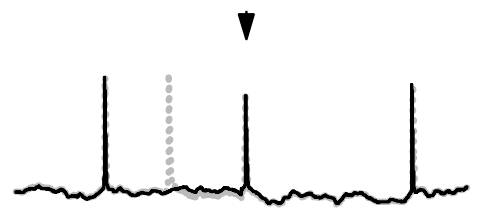

$\overline{50 \mathrm{~ms}}$

D

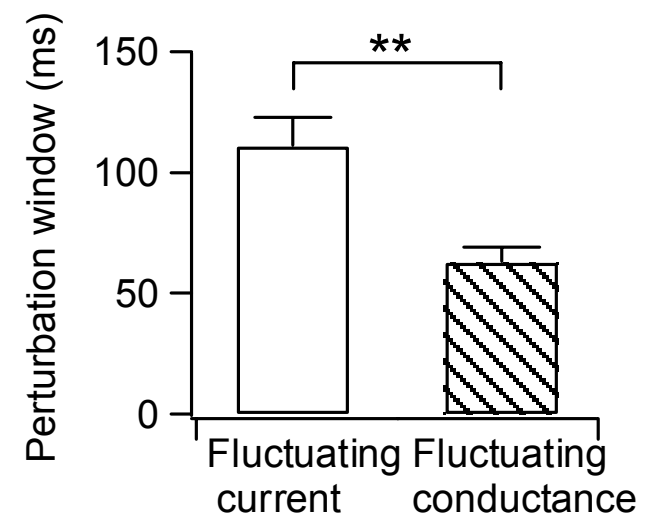


in histograms of $10 \mathrm{~ms}$ bins according to the position of the timing of spikes in the control traces. In $\mathrm{N}=10$ cells the advancement of spikes completely diminished after $20 \mathrm{~ms}$ (after the second bin), and most of the cells even after $10 \mathrm{~ms}$ (the second bin) was no significant difference (p values were larger than 0.1 ) tested with $t$-test for independent samples between every bin pairs. When data was pooled from all the cells $(\mathrm{N}=10)$ from the bins between $0-10 \mathrm{~ms}$, the range of advancement was between $0 \mathrm{~ms}$ and $5.6 \mathrm{~ms}$ with an average of $1.2 \pm 0.8 \mathrm{~ms}$.

When an EPSP resulted in a spike, it delayed other spikes in the spike train. The length of the delay depended on the position of the spike on the spike train, the presence of the preceding spikes and the underlying stimulation (Figure 16A and B). The reliability and precision of the spike train was measured when the spikes preceded the simEPSP and started $100 \mathrm{~ms}$ after the stimulus onset. Reliability was measured as the percentage of single spikes that had no pairs in the corresponding trace within $25 \mathrm{~ms}$ of presentation. Precision was calculated as a standard deviation of the time differences from the corresponding spike pairs that occurred within $25 \mathrm{~ms}$ range in the alternating trace pairs. Precision values were not significantly different with fluctuating current and fluctuating conductance stimulation ( $\mathrm{p}=0.592, \mathrm{~N}=6$ cells). Reliability values were also not different in the pairs of the experiments stimulated with fluctuating current and fluctuating conductance, and they ranged from 3 to $11 \%(\mathrm{p}=0.677, \mathrm{~N}=6)$.

To evaluate how much an EPSP-related spike perturbed the pattern of spike trains, we generated a recovery function (called Cumulative delay in spike timing; Figure 16C) from those traces in which an EPSP contributed a spike to the peak synaptic gain (simEPSP-spike). All the spikes on the control traces that could have been influenced by the simEPSP-spike were characterized by two values: their relative position to the simEPSP-spike, and a "delay", which was calculated from the delay of the corresponding spike in the spike train with simEPSP-spike. 
If a spike on the control train did not occur due to the simEPSP-spike, then its delay was calculated as a delay to the position of the next spike in the simEPSP-spike train, independent of whether that given spike was related to other spikes in the control spike train. In this way the maximum delay was controlled by the frequency. The average delay for spikes that did not occur was the reciprocal value of the frequency.

Our analysis used the notion that when the simEPSP-spike had no effect on a given spike, then the delay approached zero (Figure 16A and B marks spikes with no effect marked with arrows) and the delay was higher for spikes that were supposedly influenced by the simEPSPspike. The delay in the position of the spikes due to the simEPSP-spike monotonically decreased, as the spike occurred later in the spike train. The recovery function was calculated as the cumulative sum of delays in spike timing by the relative position of spikes and normalized to the number of spikes used for analysis. In this way the recovery function follows the time distribution of delays and it has a plateau, which means that there was a point where the simEPSP-spike did not influence the spike train anymore. (Figure 16C). The perturbation window was defined as the time where the recovery function reached $95 \%$ of the plateau value. This means that if a spike occurred after the perturbation window, the probability was only $5 \%$ that its timing was changed because of the presence of the simEPSP spike. Note: although the reliability and precision values were high (see above) they were not $100 \%$. To find the $95 \%$ of the plateau value, the recovery function was fitted with a sigmoid curve (See Experimental Methods section). The perturbation window was significantly lower in the presence of conductance (Figure 16D) $(\mathrm{p}=0.002, \mathrm{~N}=6$ cells). The value of the plateau was always lower for experiments stimulated with fluctuating conductance than that those with fluctuating current and it was related to: 
1) The higher average delays in the individual spike pairs between the control and traces with simEPSP when the cell was stimulated with fluctuating current; and/or

2) The higher number of spikes, which were suppressed by the simEPSP-spike.

Because of (2), it was expected that the loss of spikes would be higher for spike trains generated with fluctuating current (Figure 16E). Here we should also consider that as the frequency gets higher, the loss of spikes gets closer to 1 (see the changes of loss spikes when cell stimulated with conductance in Figure $14 \mathrm{C}$ ). However there may be a difference in the loss of spikes measured at lower frequencies. At lower frequencies $(3.8-5 \mathrm{~Hz})$, the loss of spikes was significantly different from the averages of $0.35 \pm 0.06$ and $0.21 \pm 0.05$ for fluctuating conductance and fluctuating current $(\mathrm{p}=0.034, \mathrm{~N}=5$ cells). The difference in the loss of spikes was not the result of the difference in the peak synaptic gain under the two conditions, which was not significantly different $(0.43 \pm 0.05$ and $0.46 \pm 0.07$, for fluctuating current and conductance, respectively, $\mathrm{N}=7$ cells, $\mathrm{p}=0.465$ ).

The width of the peak synaptic gain in the PSTH with fluctuating current and fluctuating conductance stimulation, as a measurement of spike timing precision in a cell with ongoing spiking activity. Stimulating the cell with fluctuating current generated peak synaptic gains that were $2.2 \pm 0.17$-fold longer than width of the peak synaptic gain generated with fluctuating conductance stimulation. The average width of the peak synaptic gain was $13.3 \pm 4.9 \mathrm{~ms}$ and 5.8 $\pm 2.8 \mathrm{~ms}$ for fluctuating current and fluctuating conductance, respectively ( $\mathrm{p}=0.044, \mathrm{~N}=6)$.

Refractory period was also measured, and the refractory period of spikes generated with fluctuating current was consistently higher in every experiment pair by $1.2 \pm 0.1$-fold, than refractory periods generated with fluctuating conductance The average values of refractory 
periods were $71.1 \pm 9.3$ and $62.2 \pm 10.0(\mathrm{p}=0.033, \mathrm{~N}=6)$ for experiments stimulated with fluctuating current and for experiments stimulated with fluctuating conductance, respectively.

Depending on the properties of the background synaptic activity, the cell can maintain a longer or shorter "memory" of a previous signal of how long it takes for the cell to return to its “original" spiking pattern corresponding to a given input. There are two factors contributing to the length of the perturbation, the presence of fluctuation, which causes irregularity in spiking (Figure 15) and the presence of conductance in the background (Figure 16). Our definition of perturbation included the effect of simEPSP-related spike on those spikes that did not occur due to EPSP spike and those spikes that were delayed due to the simEPSP spike. This suggests that the presence of simEPSP-spike changed the reliability of those spikes and they disappeared from the spike train due to the simEPSP-spike. As a consequence we found a connection between the way a neuron reacted to perturbation by a spike and the loss of spikes. 


\section{CHAPTER IV. DISCUSSION}

\section{Summary}

The effect of background synaptic activity was studied on EPSP-spike coupling. We considered spike timing and the regulation of synaptic gain. In the first part of the study we addressed how the background synaptic activity interacts with the passive and active properties of the cell to facilitate spike-timing precision. Ongoing synaptic activity may promote precision of spike timing in vivo, because in this in vitro system it reduced the depolarization- induced EPSP prolongation. Background synaptic conductance also reduced the increase in the EPSP rise time due to depolarization, but it did not reduce the increase in the EPSP amplitude compared to that of recorded at resting potential. In a cell with ongoing firing activity, an EPSP caused a transient increase in the frequency of discharge. The probability that an EPSP evoked a spike (peak synaptic gain) depended linearly on the amplitude of the EPSP. The time course of the peak synaptic gain was related to the EPSP rise time. The peak synaptic gain was followed by a reduced probability of firing and we examined. We examined the two possible explanations for this reduced probability of firing: (1) a limited window of reduced excitability of the cell, because of the relative refractory period and (2) or the EPSP-related spike cause a realignment of the subsequent spikes in the spike train. To analyze what happen with the spike counts, we calculated the probabilities (termed the "loss of spikes") that if a spike is generated due to the EPSP (increase in the spike count) then what the probability is this increase is not present after a longer period. We found that the peak synaptic gain was not significantly affected by the background firing rate, however the "loss of spikes" increased with the background frequency 
increase. We concluded that background frequency might provide a powerful mechanism to regulate the overall spike count: with higher frequency a single EPSP only affects the timing of spikes but not the spike count.

EPSP-related spikes can also disturb the timing of spikes; in this way a cell appears to preserve a "memory" of the previous activity in the timing of spikes. A single EPSP had a negligible effect on spike train if it is not coupled to a spike. However when the EPSP was coupled to a spike it affected the timing of spikes over a greater period. The two regulators of the length of the perturbation by an EPSP-related spike were (1) the irregularity in the spiking pattern, induced by the fluctuation in the membrane potential and (2) addition of extra conductance also had a significant effect. We also found that the longer an extra spike disturbed the timing of subsequent spikes was, the larger the probability of suppressing a spike became (loss of spikes). Based on these data we concluded that synaptic activity, which cause a fluctuation in the membrane potential and conductance regulates efficacy of EPSP-spike coupling. The effect of conductance is double: (1) increases the precision of spike timing generated by a single EPSP and (2) limits the interaction between the spikes in the train. As a consequence, the capacity to transmit information by its spike train output may be regulated with the presence of background synaptic activity.

\section{Limitations of the experimental design}

Under the present experimental conditions the ongoing synaptic background activity was recreated with injection of point conductances into the pyramidal cells. To study the effect of synaptic activity, unitary current transients were collected at the somata of pyramidal cells from 
paired recordings between pyramid->pyramidal cells and fast spiking cell->pyramidal cells (Galarreta and Hestrin, 1998) and these currents were used to generate unitary conductance transients, which were injected into the soma of other pyramidal cells.

In the experimental design we assumed a very simplified model of the cell. (1) Pyramidal cells have a very elaborate morphology and as a consequence they are not electronically compact, but we did not deal with that fact. (2) Synaptic conductances are widely distributed along the dendrites, but we only studied their interaction on the soma. (3) We assumed that synaptic activity is balanced, that is the ration of excitatory and inhibitory conductance is more or less constant, and (4) their temporal distribution resembles an uncorrelated Poisson process.

According to cable theory, the transmembrane current will attenuate in amplitude and it will have a slower kinetics with distance on a neuron containing passive membrane properties only (Rall, 1989). The level of attenuation will depend on the traveling distance from the site of origination of the current transient, and the larger the ratio of the membrane resistivity to the intracellular resistivity the smaller the attenuation is. We should also consider that voltage attenuation on the dendrites of pyramidal cells depends on the active conductances distributed along the dendrite, such as synaptic activity combined with voltage dependent conductances, and membrane properties (membrane resistivity and capacitance). These properties are not uniform along the cell; for example the membrane resistivity is significantly lower in the dendrites than in the soma (Stuart and Spruston, 1998).

When studying the effect of attenuation of voltage signals from the soma to the distal dendrite under our experimental conditions, it is uncertain how much the dendritic resting voltage dependent conductances interact with the injection of conductance at the soma. 
Injection of conductance into the soma does not affect the attenuation of voltage transients traveling from the soma to the distal part of the dendritic tree. However the transient is affected by the dendritic conductances, which occur as leak compared to the conductance on the soma (Spruston et al., 1993). Moreover, when stimulated with fluctuating conductance, the attenuation of voltage transient is expected to be higher then when stimulated with an invariable step current, because the level of attenuation also depends on the frequency components of the membrane potential fluctuation (Spruston et al., 1993). Since we injected fluctuating conductance, these observations suggest that our experimental design allowed us to study the activity of somatic conductances and the dendrites have relatively small contribution.

However we should consider here that there are active conductances on the proximal dendrites, which may interact with somatic conductances, the level of which in turn depends on the membrane potential. Basal dendrites may be more compact electronically than apical dendrite, which may influence the somatic processes. They also contain NMDA receptors in a higher ratio compared to the ration on the apical dendrite of the pyramidal cell. Considering the facts that slices contain ambient glutamate that may generate a tonic current from the activation of NMDA receptors (Sah et al., 1989), NMDA receptors may serve as a powerful modifier of the somatic activity (Schiller et al., 2000). To evaluate the space clamping by the dynamic clamp and the somatic and dendritic interaction in the EPSP-spike coupling, further investigations are necessary, for example using models.

Other problems may occur due to series resistance between the recording electrode and the cell. When it was possible we used the double electrode recording for this reason. Because the input resistance of pyramidal cell is low and we even made it lower by injecting conductance, measurement of the EPSP parameters could be affected by the series resistance. The effect of 
series resistance is greater on the measurement of the amplitude of synapses that are closer to the soma. The level of distortion is bigger with larger synaptic conductances (Spruston et al., 1993). However, the effect of conductance resulted in very similar values when the ratio of the amplitude measured at depolarized membrane potentials and the amplitude measured at resting potentials was compared between simulated EPSPs and evoked EPSPs. The evoked EPSP were probably originated from several hundred $\mu \mathrm{m}$ from the soma and they also had a wider range of rise time kinetics, as well as wide ranges of amplitude distribution. Similar conclusions could be derived about the comparison of normalized rise times (measured at depolarized potentials/resting membrane potential) between the samples of simulated EPSPs and evoked EPSPs. These latter would be affected in a similar way independently from the location of EPSP. Another simplification was that when the conductance waves were generated, IPSCs and EPSCs of only the same size were used. However, unitary conductances, measured on the soma may have a variability in their amplitude and time course, moreover they are prone to synaptic depression when the cell is continuously active. In our experiments we did not consider the presence of synaptic depression, and even with evoked EPSP, the waiting period between trials was carefully chosen to prevent the depression of the synaptic activity. The variability of EPSPs and IPSP in vivo may generate a fluctuation in the membrane potential with larger variability. (compare Azouz and Gray (1999a to trace on Figure 5.) However, the presence of tonic conductance could be modeled with the dynamic clamp system and have shown a powerful effect both on influencing the precision of spike timing evoked by a single EPSP and limiting the interaction between spikes in the spike train. And we have also shown that fluctuation was enough to generate a quantitatively different interaction between spikes in the spike train. 
Further studies are necessary to study that a combination of a more realistic fluctuation in the membrane potential and tonic conductance would make a qualitative or quantitative difference in the properties of EPSP spike coupling.

\section{Mechanism for prevention of prolongation}

It has been suggested that pyramidal cells cannot transmit information about the timing of presynaptic activity because of the presence of the prolongation of EPSPs at membrane potential values close to firing threshold when depolarized with a current pulse (Fricker and Miles, 2000, Stuart and Sakmann, 1995, Andreasen and Lambert, 1999). The prolongation becomes highly sensitive to changes in the membrane potential just below the spike threshold (Figure 8B; Stuart and Sakmann, 1995, Andreasen and Lambert, 1999), and this prevents the precise time locking of spike generation to the EPSP onset. The prolongation of the EPSP half width could be explained by the presence of a noninactivating sodium current on the soma and proximal apical dendrite of the pyramidal cell (Stafstrom et al, 1984, Stuart and Sakmann, 1995, Deisz et al., 1991, Fricker and Miles, 2000) that has a low, but significant density in the membrane. These currents are activated in a subthreshold voltage range (Alzheimer et al., 1993). The estimated density of persistent sodium currents is ranging from $0.25 \%$ to $1-2 \%$ of the overall $\mathrm{Na}^{+}$-current (French et al., 1990, Alzheimer et al., 1993), but this amount is enough to depolarize the membrane potential in the presence of excitatory inputs in a greater amount compared to the depolarization of a passive membrane.

The persistent sodium currents on the distal dendrites of CA1 pyramidal cells (Andreasen and Lambert, 1999) and layer 5 neocortical pyramidal neurons (Schwindt and Crill, 1995, 
Gonzalez-Burgos and Barrionuevo, 2001) might also participate in the prolongation of the EPSP half width and increase in the amplitude during depolarization. However, the prolongation of the EPSP half width by the activation of dendritic persistent sodium currents might be masked by potassium conductances (Andreasen and Lambert, 1999, Gonzalez-Burgos and Barrionuevo, 2001). In one study (Lipowsky et al., 1996) only the persistent sodium currents that were responsible for the prolongation of EPSP half width on CA1 pyramidal cells originated from the dendrites. We should mention here that other ion channels also participate in the depolarizationinduced prolongation of EPSP half width and relative increase in the EPSP amplitude, compared to that obtained at resting potential. One cause of this result maybe the low threshold activated calcium channel on the dendrites of CA1 pyramidal cells (Gillessen and Alzheimer, 1997). Low threshold Ca-channels can also cause an increase in the prolongation of EPSP half width with depolarization (Gonzalez-Burgos and Barrionuevo, 2001) when the potassium conductances are blocked in the dendrites. Recruitment of synaptic NMDA receptors by depolarization was also reported as a possible mechanism for the prolongation of EPSP (Schwindt and Crill, 1995, Thomson, 1997) because depolarization can remove the magnesium block that is present at more hyperpolarized conditions and also glutamate was shown to be present in the slice in such a concentration that can activate NMDA receptors on the dendrites (Sah et al., 1989).

Our results are in agreement with those results describing the participation of the persistent sodium currents in the depolarization induced prolongation, because TTX restores the simulated EPSP parameters obtained at near threshold conditions to the values measured at resting potential with step current as a background activity. Similarly, when the cells are depolarized by background conductance, which diminished the prolongation of EPSP half width but not the EPSP amplitude increase with depolarization, TTX reduces the EPSP amplitude to 
the value obtained at resting potential. We did not pursue further the underlying mechanism of how conductance reduces the prolongation of EPSP half width due to depolarization by a current pulse because of the lack of selective persistent sodium current blockers. One study reported that phenytoin, an antiepileptic drug selectively blocks persistent sodium currents on CA1 pyramidal cells (Fricker and Miles, 2000). Application of phenytoin (see in Mechanism of prolongation part in the Results section) on layer 5 pyramidal cells reduced the prolongation of EPSP half width and the increase in the EPSP amplitude when the cell was depolarized from resting potential to near threshold. However, it also affects spike threshold and size, which makes it hard to compared the EPSP parameters at a given membrane potential. Thus phenytoin is not the right blocker to study the role of persistent sodium currents. In summary, the results with a putative blocker of persistent sodium channels are inconclusive, we can only hypothesize the mechanism underlying the reduction of EPSP half width when cells depolarized with background conductance. An increase in the input conductance reduces the membrane potential displacement due to the activation of persistent sodium currents at near spike threshold potentials. The transient and persistent voltage dependent sodium currents, however, are activated with depolarization, independent from the level of the background conductances. Thus, the amplitude of EPSP increases with the depolarization, even without the presence of the prominent half width increase. Alternatively, only transient sodium currents could be responsible for the increase in EPSP amplitudes and persistent sodium currents could be responsible for the prolongation of EPSP half width induced by depolarization from resting potential. 


\section{How much conductance is sufficient to improve the precision of spike timing?}

It is well established that the dynamics of network activity represented in the firing of a single neuron (Steriade, 2001). It is also known the level of network activity depends on the "brain-state", e.g. sleep and vigilance (Steriade et al., 2001, Steriade, 2001), level of anesthesia (Armstrong-James and George, 1988) or attention (Martinez-Trujillo and Treue, 2002). Although ongoing synaptic activity can reduce a cell's input resistance by up to $70 \%-80 \%$ (Pare et al., 1998, Destexhe and Pare, 1999), we were interested in what might be a minimum value to prevent the depolarization induced prolongation of EPSP half width (Figure 9C). We found that about a $35 \%$ reduction in the input resistance by tonic conductance injection is sufficient to diminish the prolongation of EPSP half width induced by depolarization with a current pulse. We also found that addition of a subthreshold fluctuation does not change significantly the prolongation and the prevention of prolongation by background conductance (Figure 10).

There are two major sources of conductance in cells with ongoing spiking activity in vivo: synaptic input, and conductances that cause activation and are related to the spike generation. $\mathrm{Na}^{+}$- and $\mathrm{K}^{+}$-channels are activated during an action potential and they could generate a prominent conductance on the soma and the proximal part of the axon (Cole and Curtis, 1939). This conductance may decrease the amplitude of an EPSP not related to the spike itself by up to $30 \%$ of its original value. The decrease occurs within the window starting before the spike and ending 10-15 ms after the spike (Hausser et al., 2001). The spike related conductance, added to the level of conductance from the synaptic activity may provide a further improvement in the precision of spike timing. This may be because at that level of conductance, adding an extra conductance still lowers the EPSP half width (Figure 10B). With data from Figure $11 \mathrm{C}$, we found the spike time window was reduced 1.5 -fold more with tonic conductance 
stimulation compared to the reduction of the EPSP half width. This suggests that spiking activity may provide an additional improvement in the spike timing precision, when cells fires with higher frequency vs the cell fires with a lower frequency. Taken together in cells where the ongoing background synaptic activity generates a high frequency of firing, the precision of EPSP-spike would be expected to be higher then the presence of conductance but without the firing. However, we did not find significant correlation between the frequency of discharge and the width of the peak synaptic gain. The reason could be that values of evoked EPSP rise time fall into a relatively wide range and peak synaptic gain was strongly dependent upon the EPSP rise time. There is a difference between the width of peak synaptic gains when stimulated with fluctuating current or fluctuating conductance: the width of the peak synaptic gain generated with fluctuating current stimulation was $2.2 \pm 0.18$-fold longer than the width of peak synaptic gain on histograms generated with fluctuating current.

A recent study applying whole cell patch clamp recording techniques reported that the level of ongoing spiking activity is low in awake animals (Margrie et al., 2002). Data from anesthetized animals also support this (Margrie et al., 2002, Moore and Nelson, 1998, Zhu and Connors, 1999). As a consequence, conductance from background synaptic activity cannot be high. Margrie and co-workers (2002) found that the frequency of firing in the somatosensory cortex of an awake rat is less than $1 \mathrm{~Hz}$. This contradicts studies employing wide variety of techniques, which have reported frequency values of $5-40 \mathrm{~Hz}$ in awake animals (Hubel and Wiesel, 1959, Steriade et al., 2001, Mountcastle et al., 1969, Azouz et al., 1997). There is no explanation yet for this discrepancy. We might assume that there are differences between cortical areas in the level of ongoing spiking frequency. All the low values were recorded from the somatosensory cortex, and higher values were mainly from the visual cortical areas (except data 
from Mountcastle et al.; 1969). Animals were kept in a stereotaxic device during the recording, which prevents the constant stimulation from the environment that could be important for the correct functioning of somatosensory cortex. Further studies are necessary to decide what are the physiological values for the level of the spontaneous activity in the cortex.

\section{Refractory period and the effect of a single spike on the spike timing}

Neuronal refractory period occurs immediately after a spike. As a consequence, the probability of firing at a given moment depends not only on the stimulation, but also on the timing of the preceding spike in the train. Thus, refractory period restricts maximal firing frequency. This would limit the neuron's capacity for transmission of information in a coding system that conveys information based on the frequency of spiking. For this reason, in earlier studies the effect refractory period had to be removed from the observed spike train in order to estimate the stimulus related spike data from the instantaneous firing rate (Gray, 1967). Although we know more about the ionic mechanisms underlying the refractory period (Hodgkin and Huxley, 1952, Schwindt et al., 1988b, Schwindt et al., 1988a, Schwindt et al., 1989, Spain et al., 1991, Schwindt et al., 1992, Sah and Faber, 2002), its role in neuronal integration is not well understood.

After a neuron fires an action potential, the cell preserves a "memory" of that spike by resetting the membrane potential in the soma and proximal dendrites. With this reset, the membrane potential ends up further away from the spike initiation threshold. Depending on how long the interval is before the next spikes, spikes can accumulate into the spike trains with different consequences (Abeles, 1991). (1) During the early refractory phase only a higher level 
of network activity can create a spike. A continuous high level of network activity creates a regular spiking, limited only by the length of the absolute refractory period. (2) A spike may arrive during an "intermediate zone", after the early refractory period (the definition relates to the fact, that the average membrane potential is still lower than the steady state membrane potential). (3) A spike can arrive when the membrane potential reached the steady state and a previous spike does not effect its timing, but stimulation does.

Our results showed on Figure 15 and Figure 16 that the length these intervals depends on the stimulation itself. A cell stimulated with a step current behaves like all the spikes arrive during the early refractory period of the previous spike. Any perturbation due to an "extra" spike accumulated in the timing of the following spikes. As a consequence the net synaptic gain is low when the cell fires with regular interspike interval. However, it is likely that a spike will not perturb the timing of other spikes, if it arrives during the time, when the cell's membrane potential reached its steady state. And, when the frequency of spiking is high enough, than most of the spikes arrive during the "intermediate zone" of the previous spike, and the timing of spikes and the probability of accumulation of a spike in the spike train will depend not only on the stimulation but the timing of the previous spikes. The presence of fluctuation in this case is important, because it allows the membrane potential to be "reset", and it acts by shortening of the effect of a single spike on the subsequent spikes.

To analyze what happens when an EPSP arrives at a cell with ongoing activity, we defined the perturbation window. The cell's perturbation window was measured by how an actively spiking cell reacts to the application of an extra spike. The measure contained two parameters: the maximal latency of all the subsequent spikes that showed a jitter in their timing compared to the timing of their pairs on the control spike train. In case the spike on the test train 
did not occur, the maximal latency was the reciprocal value of the frequency of firing. EPSP creates only a very short perturbation in the timing of spikes and only a spike due to the EPSP could create a longer memory extending tens of milliseconds.

Although there was no significant difference in the refractory period when cells were stimulated with fluctuating current vs. fluctuating conductance, we found that the presence of synaptic conductance reduced the impact of a single spike on the spike train. This could be because the length of the perturbation window depends on two possible factors: the precision of EPSP-spike coupling and the interaction of the refractory period with the background spiking frequency. Although there was no difference between the reliability and precision of spike generation due to the fluctuating conductance and fluctuating current, we found a small but significant difference in the width of the peak of cells' PSTHs, thus the precision of EPSP-spike coupling was higher, when the ongoing spiking activity was generated with fluctuating conductance compared to that generated with fluctuating current.

Adding an extra conductance reduced the size of the afterhyperpolarization (Table 1 in Experimental Methods). The mechanism for the reduction of the refractory period is similar to the way the conductance reduces or prevents prolongation. If the input resistance is lower and the currents underlying the refractory period cannot displace the membrane potential as much, then in a cell with no background conductances (Table 1). As a consequence, the membrane potential stays closer to the firing threshold. We should note here this mechanism does not affect the size or timing of the absolute refractory period, which is due to the sodium channel inactivation Hodgkin and Huxley, 1952).

Thus the presence of the background activity, together with the refractory period provided a possible mechanism for regulation of the accumulation of spikes in the spike train, 
independently from the peak synaptic gain. Cells with shorter perturbation window accumulate more spikes, but not affecting the peak synaptic gain. A larger amount of conductance shortens the effect of the cell's past activity on the timing of spikes, allowing more independence between single spikes to transmit information. Intuitively, if a perturbation arrives that changes the structure of the spike train by delaying spikes, the sooner the spike train "gets back" into the "intended" spike train mode, the higher number of spikes can be accumulated in the spike train. In this way, a larger amounts of background synaptic activity allows more rapid regulation of synaptic efficacy. Conversely, when synaptic activity is low, the cell is in an integrative mode, and the efficacy is high.

\section{Concluding remarks}

Network activities are thought to have a profound impact on several properties of the cells. The network activity in the cortex arises from the thalamus, the interaction between thalamo-cortical synapses, and cortico-cortical synapses. The level of network synaptic activity is not constant but controlled by other structures, e.g., subcortical modulatory structures (Steriade, 2001). In this project we explored how the network activity may regulate the integrative properties of pyramidal cells, and whether a higher levels of background synaptic activity may allow for a more efficient information transmission in the cortex.

The presence of background synaptic conductances due to the network activity was suggested as something that influences gain modulation (Chance et al., 2002, Prescott, 2003). In those studies, synaptic integration from many active synapses was considered and the authors

concluded that level of network activity allows for a dynamic modulation of the integration of all 
asynchronous inputs were assumed to arrive with a constant rate for a longer time period. Our experimental approach was different, because we studied the influence of the network activity in the context of a single synaptic input, which is a transient signal. Based on this paradigm, we were able to show experimentally that network activity may regulate precision of spike timing. Further, the efficacy of EPSP-spike coupling is related to the precision of timing. Activation of a single input increases the probability of spike, however the probability that a number of spikes accumulated in the spike train also depends on the network activity, and the refractory period of the cell.

In this study we chose to examine rather the role of tonic conductance and kept the fluctuation in the membrane potential constant. A further step will be to investigate experimentally the role of the activating synaptic conductance related fluctuation in the membrane potential in the integration of a single synaptic input. A recent study by Rudolph and Destexhe (Rudolph and Destexhe, 2003) using a computational model and parameters measured in vivo and in vitro, also concluded that higher level of background activity may allow a fast regulation of synaptic efficacy because of the presence of high fluctuation in the membrane potential. They argue that tonic conductance reduces the difference between the impacts of the synapses located more distally or more proximally, but the fluctuating background conductance allowed a "boosting" of the dendritic input and increased the probability that the dendritic input reached the soma. Their model predicts that the highest synaptic efficacies can be obtained with the highest level of background activity and argue that background activity interacts with channels on the dendrites. Our experimental system could be used to test these ideas experimentally. The two mechanisms, (1) the effect of tonic conductance on the precision of EPSP-spike coupling, and (2) the fluctuating conductance, which assumed to increase the 
efficacy of EPSP-spike coupling would determine the properties of information transmission between cells in the neuronal network. 


\section{LIST OF REFERENCES}


Abbott, L. F., Varela, J. A., Sen, K. and Nelson, S. B. (1997). Synaptic depression and cortical gain control. Science, 275, 220-4.

Abeles, M. (1982a) Local cortical circuits: an electrophysiological study. Springer-Verlag; Berlin, New York.

Abeles, M. (1982b). Role of the cortical neuron: integrator or coincidence detector? Isr J Med Sci, 18, 83-92.

Abeles, M. (1982c). Quantification, smoothing, and confidence limits for single-units' histograms. J Neurosci Methods, 5, 317-25.

Abeles, M. (1991) Corticonics. Neural circuits of the cerebral cortex. Cambridge University Press; Cambridge, England.

Adrian, E. D. (1926). The impulses produced by sensory nerve endings. Part I. J Physiol, 61, 4972.

Agmon, A. and Connors, B. W. (1991). Thalamocortical responses of mouse somatosensory (barrel) cortex in vitro. Neuroscience, 41, 365-79.

Alzheimer, C., Schwindt, P. C. and Crill, W. E. (1993). Modal gating of Na+ channels as a mechanism of persistent $\mathrm{Na}+$ current in pyramidal neurons from rat and cat sensorimotor cortex. J Neurosci, 13, 660-73.

Anderson, J. S., Carandini, M. and Ferster, D. (2000). Orientation tuning of input conductance, excitation, and inhibition in cat primary visual cortex. J Neurophysiol, 84, 909-26.

Andreasen, M. and Lambert, J. D. (1999). Somatic amplification of distally generated subthreshold EPSPs in rat hippocampal pyramidal neurones. J Physiol, 519 Pt 1, 85-100.

Arieli, A., Sterkin, A., Grinvald, A. and Aertsen, A. (1996). Dynamics of ongoing activity: explanation of the large variability in evoked cortical responses. Science, 273, 1868-71. 
Armstrong-James, M. and George, M. J. (1988). Influence of anesthesia on spontaneous activity and receptive field size of single units in rat Sm1 neocortex. Exp Neurol, 99, 369-87.

Azouz, R. and Gray, C. (1999a). Cellular mechanisms contributing to response variability of cortical neurons in vivo. J Neurosci, 19, 2209-23.

Azouz, R., Gray, C. M., Nowak, L. G. and McCormick, D. A. (1997). Physiological properties of inhibitory interneurons in cat striate cortex. Cereb Cortex, 7, 534-45.

Bair, W. and Koch, C. (1996). Temporal precision of spike trains in extrastriate cortex of the behaving macaque monkey. Neural Comput, 8, 1185-202.

Barlow, H. B. (1972). Single units and sensation: a neuron doctrine for perceptual psychology? Perception, 1, 371-94.

Berman, N. J., Douglas, R. J., Martin, K. A. and Whitteridge, D. (1991). Mechanisms of inhibition in cat visual cortex. J Physiol, 440, 697-722.

Bernander, O., Douglas, R. J., Martin, K. A. and Koch, C. (1991). Synaptic background activity influences spatiotemporal integration in single pyramidal cells. Proc Natl Acad Sci U S A, 88, 11569-73.

Bernander, O., Koch, C. and Douglas, R. J. (1994). Amplification and linearization of distal synaptic input to cortical pyramidal cells. J Neurophysiol, 72, 2743-53.

Bliss, T. V. and Lomo, T. (1973). Long-lasting potentiation of synaptic transmission in the dentate area of the anaesthetized rabbit following stimulation of the perforant path. J Physiol, 232, 331-56.

Borg-Graham, L. J., Monier, C. and Fregnac, Y. (1998). Visual input evokes transient and strong shunting inhibition in visual cortical neurons. Nature, 393, 369-73. 
Buracas, G. T., Zador, A. M., DeWeese, M. R. and Albright, T. D. (1998). Efficient discrimination of temporal patterns by motion-sensitive neurons in primate visual cortex. Neuron, 20, 959-69.

Calvin, W. H. and Stevens, C. F. (1968). Synaptic noise and other sources of randomness in motoneuron interspike intervals. J Neurophysiol, 31, 574-87.

Castro-Alamancos, M. A., Donoghue, J. P. and Connors, B. W. (1995). Different forms of synaptic plasticity in somatosensory and motor areas of the neocortex. J Neurosci, 15, 5324-33.

Chance, F. S., Abbott, L. F. and Reyes, A. D. (2002). Gain modulation from background synaptic input. Neuron, 35, 773-82.

Cole, K. S. and Curtis, H. J. (1939). Electric impedance of the squid giant axon during activity. J. Gen. Physiol. 22 649-70.

Cope, T. C., Fetz, E. E. and Matsumura, M. (1987). Cross-correlation assessment of synaptic strength of single Ia fibre connections with triceps surae motoneurones in cats. J Physiol, $390,161-88$.

de Ruyter van Steveninck, R. R., Lewen, G. D., Strong, S. P., Koberle, R. and Bialek, W. (1997). Reproducibility and variability in neural spike trains. Science, 275 , 1805-8.

deCharms, R. C. and Zador, A. (2000). Neural representation and the cortical code. Annu Rev Neurosci, 23, 613-47.

Deisz, R. A., Fortin, G. and Zieglgansberger, W. (1991). Voltage dependence of excitatory postsynaptic potentials of rat neocortical neurons. J Neurophysiol, 65, 371-82.

Destexhe, A. and Pare, D. (1999). Impact of network activity on the integrative properties of neocortical pyramidal neurons in vivo. J Neurophysiol, 81, 1531-47. 
Destexhe, A., Rudolph, M., Fellous, J. M. and Sejnowski, T. J. (2001). Fluctuating synaptic conductances recreate in vivo-like activity in neocortical neurons. Neuroscience, 107, 1324.

Engel, A. K., Konig, P., Kreiter, A. K., Schillen, T. B. and Singer, W. (1992). Temporal coding in the visual cortex: new vistas on integration in the nervous system. Trends Neurosci, $15,218-26$.

Feldman, D. E. (2000). Timing-based LTP and LTD at vertical inputs to layer II/III pyramidal cells in rat barrel cortex. Neuron, 27, 45-56.

Fetz, E. E. and Gustafsson, B. (1983). Relation between shapes of post-synaptic potentials and changes in firing probablity of cat motoneurones. Journal of Physiology, 341, 387-410.

Fetz, E. E., Toyama, K. and Smith, W. (1991) Synaptic interactions between cortical neurons. in Cerebral Cortex. vol 9. Plenum Press; New York. pp. 1-49.

French, C. R., Sah, P., Buckett, K. J. and Gage, P. W. (1990). A voltage-dependent persistent sodium current in mammalian hippocampal neurons. J Gen Physiol, 95, 1139-57.

Fricker, D. and Miles, R. (2000). EPSP amplification and the precision of spike timing in hippocampal neurons. Neuron, 28, 559-69.

Frostig, R. D., Frostig, Z. and Harper, R. M. (1990a). Recurring discharge patterns in multiple spike trains. I. Detection. Biol Cybern, 62, 487-93.

Frostig, R. D., Frysinger, R. C. and Harper, R. M. (1990b). Recurring discharge patterns in multiple spike trains. II. Application in forebrain areas related to cardiac and respiratory control during different sleep-waking states. Biol Cybern, 62, 495-502.

Galarreta, M. and Hestrin, S. (1998). Frequency-dependent synaptic depression and the balance of excitation and inhibition in the neocortex. Nat Neurosci, 1, 587-94. 
Galarreta, M. and Hestrin, S. (1999). A network of fast-spiking cells in the neocortex connected by electrical synapses. Nature, $402,72-5$.

Galarreta, M. and Hestrin, S. (2001). Spike transmission and synchrony detection in networks of GABAergic interneurons. Science. 292, 2295-9.

Gaumond, R. P., Molnar, C. E. and Kim, D. O. (1982). Stimulus and recovery dependence of cat cochlear nerve fiber spike discharge probability. J Neurophysiol, 48, 856-73.

Gillessen, T. and Alzheimer, C. (1997). Amplification of EPSPs by low Ni(2+)- and amiloridesensitive $\mathrm{Ca} 2+$ channels in apical dendrites of rat $\mathrm{CA} 1$ pyramidal neurons. $\mathrm{J}$ Neurophysiol, 77, 1639-43.

Gonzalez-Burgos, G. and Barrionuevo, G. (2001). Voltage-gated sodium channels shape subthreshold EPSPs in layer 5 pyramidal neurons from rat prefrontal cortex. $\mathrm{J}$ Neurophysiol, 86, 1671-84.

Gray, P. R. (1967). Conditional probability analyses of the spike activity of single neurons. Biophysical Journal, 7, 759-77.

Gustafsson, B. and McCrea, D. (1984). Influence of stretch-evoked synaptic potentials on firing probability of cat spinal motoneurones. J Physiol, 347, 431-51.

Gutfreund, Y., Yarom, Y. and Segev, I. (1995). Subthreshold oscillations and resonant frequency in guinea-pig cortical neurons: physiology and modelling. J Physiol, 483, 621-40.

Gutnick, M. J., Connors, B. W. and Prince, D. A. (1982). Mechanisms of neocortical epileptogenesis in vitro. J Neurophysiol, 48, 1321-35.

Hausser, M., Major, G. and Stuart, G. J. (2001). Differential shunting of EPSPs by action potentials. Science, 291, 138-41. 
Heggelund, P. and Albus, K. (1978). Response variability and orientation discrimination of single cells in striate cortex of cat. Exp Brain Res, 32, 197-211.

Hessler, N. A., Shirke, A. M. and Malinow, R. (1993). The probability of transmitter release at a mammalian central synapse. Nature, 366, 569-72.

Sah, P., Hestrin, S. and Nicoll, R. A. (1989). Tonic activation of NMDA receptors by ambient glutamate enhances excitability of neurons. Science, 246, 815-18.

Hirsch, J. A., Alonso, J. M., Reid, R. C. and Martinez, L. M. (1998). Synaptic integration in striate cortical simple cells. J Neurosci, 18, 9517-28.

Ho, N. and Destexhe, A. (2000). Synaptic background activity enhances the responsiveness of neocortical pyramidal neurons. J Neurophysiol, 84, 1488-96.

Hodgkin, A. L. and Huxley, A. F. (1952). A qualitative description of membrane current and its application to conduction and excitation in nerve. J Physiol, 117, 500-44.

Hoffman, D. A., Magee, J. C., Colbert, C. M. and Johnston, D. (1997). K+ channel regulation of signal propagation in dendrites of hippocampal pyramidal neurons. Nature, 387, 869-75.

Hubel, D. H. (1959). Single unit activity in striate cortex of unrestrained cats. J. Physiol, 147, 226-38.

Hubel, D. H. and Wiesel, T. N. (1959). Receptive fields of single neurones in the cat's striate cortex. J Physiology, 148, 574-91.

Kirkwood, P. A. and Sears, T. A. (1982). The effects of single afferent impulses on the probability of firing of external intercostal motoneurones in the cat. J Physiol, 322, 31536.

Konig, P., Engel, A. K. and Singer, W. (1996). Integrator or coincidence detector? The role of the cortical neuron revisited. Trends Neurosci, 19, 130-7. 
Kumar, S. S. and Huguenard, J. R. (2001). Properties of excitatory synaptic connections mediated by the corpus callosum in the developing rat neocortex. J Neurophysiol, 86 , 2973-85.

Lampl, I., Reichova, I. and Ferster, D. (1999). Synchronous membrane potential fluctuations in neurons of the cat visual cortex. Neuron, 22, 361-74.

Lipowsky, R., Gillessen, T. and Alzheimer, C. (1996). Dendritic Na+ channels amplify EPSPs in hippocampal CA1 pyramidal cells. Journal of Neurophysiology, 76, 2181-91.

Maccaferri, G. and Dingledine, R. (2002). Control of feedforward dendritic inhibition by NMDA receptor-dependent spike timing in hippocampal interneurons. J Neurosci, 22, 5462-72.

Magee, J. C. (1998). Dendritic hyperpolarization-activated currnets modify the integrative properties of hippocampla CA1 pyramidal neurons. Journal of Neuroscinece, 18, 761324.

Magee, J. C. (1999). Dendritic Ih normalizes temporal summation in hippocampal CA1 neurons. Nature Neuroscience, 2, 508-14.

Mainen, Z. F. and Sejnowski, T. J. (1995). Reliability of spike timing in neocortical neurons. Science, 268, 1503-6.

Margrie, T. W., Brecht, M. and Sakmann, B. (2002). In vivo, low-resistance, whole-cell recordings from neurons in the anaesthetized and awake mammalian brain. Pflugers Arch, 444, 491-8.

Markram, H., Lubke, J., Frotscher, M. and Sakmann, B. (1997). Regulation of synaptic efficacy by coincidence of postsynaptic APs and EPSPs. Science, 275, 213-5.

Martinez, L. M., Alonso, J. M., Reid, R. C. and Hirsch, J. A. (2002). Laminar processing of stimulus orientation in cat visual cortex. J Physiol, 540, 321-33. 
Martinez-Trujillo, J. and Treue, S. (2002). Attentional modulation strength in cortical area MT depends on stimulus contrast. Neuron, 35, 365-70.

Matsumura, M., Chen, D., Sawaguchi, T., Kubota, K. and Fetz, E. E. (1996). Synaptic interactions between primate precentral cortex neurons revealed by spike-triggered averaging of intracellular membrane potentials in vivo. J Neurosci, 16, 7757-67.

McCormick, D., Connors, B., Lighthall, J. and Prince, D. (1985). Comparative electrophysiology of pyramidal and sparsely spiny stellate neurons of the neocortex. J Physiol, 54, 782-806.

Mechler, F., Victor, J. D., Purpura, K. P. and Shapley, R. (1998). Robust temporal coding of contrast by V1 neurons for transient but not for steady-state stimuli. J Neurosci, 18, 658398 .

Monier, C., Chavane, F., Baudot, P., Graham, L. J. and Fregnac, Y. (2003). Orientation and direction selectivity of synaptic inputs in visual cortical neurons: a diversity of combinations produces spike tuning. Neuron, 37, 663-80.

Moore, C. I. and Nelson, S. B. (1998). Spatio-temporal subthreshold receptive fields in the vibrissa representation of rat primary somatosensory cortex. J Neurophysiol, 80, 2882-92.

Mountcastle, V. B., Talbot, W. H., Sakata, H. and Hyvarinen, J. (1969). Cortical neuronal mechanisms in flutter-vibration studied in unanesthetized monkeys. Neuronal periodicity and frequency discrimination. J Neurophysiol, 32, 452-84.

Nelson, S., Toth, L., Sheth, B. and Sur, M. (1994). Orientation selectivity of cortical neurons during intracellular blockade of inhibition. Science, 265, 774-7.

Nowak, L. G., Sanchez-Vives, M. V. and McCormick, D. A. (1997). Influence of low and high frequency inputs on spike timing in visual cortical neurons. Cereb Cortex, 7, 487-501. 
Pare, D., Shink, E., Gaudreau, H., Destexhe, A. and Lang, E. J. (1998). Impact of spontaneous synaptic activity on the resting properties of cat neocortical pyramidal neurons In vivo. $\mathrm{J}$ Neurophysiol, 79, 1450-60.

Pei, X., Wilkens, L. and Moss, F. (1996). Noise-mediated spike timing precision from aperiodic stimuli in an array of Hodgkin-Huxley-type neurons. Phys Rev Letters, 77, 4679-82.

Perkel, D. H., Gerstein, G. L. and Moore, G. P. (1967). Neuronal spike trains and stochastic point processes. I. The single spike train. Biophys J, 7, 391-418.

Poliakov, A. V., Powers, R. K. and Binder, M. D. (1997). Functional identification of the inputoutput transforms of motoneurones in the rat and cat. J Physiol, 504, 401-24.

Poliakov, A. V., Powers, R. K., Sawczuk, A. and Binder, M. D. (1996). Effects of background noise on the response of rat and cat motoneurones to excitatory current transients. J Physiol, 495, 143-57.

Prescott, S. A., De Koninck, Y (2003). Gain control of firing rate by shunting inhibition: Roles of synaptic noise and dendritic saturation. PNAS, 100, 2076-81.

Prut, Y., Vaadia, E., Bergman, H., Haalman, I., Slovin, H. and Abeles, M. (1998).

Spatiotemporal structure of cortical activity: properties and behavioral relevance. J Neurophysiol, 79, 2857-74.

Rall, W. (1989). Cable theory for dendritic neurons. In Methods in neuronal modeling (Eds, Koch, C. and Segev, I.) MIT Press; Cambridge, MA pp. 9-62.

Reyes, A. D. and Fetz, E. E. (1993). Effects of transient depolarizing potentials on the firing rate of cat neocortical neurons. J Neurophysiol, 69, 1673-83.

Rose, J., Brugge, J., DJ, A. and JE, H. (1967). Phase-locked response to low-frequency tones in single auditory nerve fibers of the squirrel monkey. J Neurophysiol, 30, 769-93. 
Rudolph, M. and Destexhe, A. (2001a). Correlation detection and resonance in neural systems with distributed noise sources. Phys Rev Lett, 86, 3662-5.

Rudolph, M. and Destexhe, A. (2001b). Do neocortical pyramidal neurons display stochastic resonance? J Comput Neurosci, 11, 19-42.

Rudolph, M. and Destexhe, A. (2003). A fast-conducting, stochastic integrative mode for neocortical neurons in vivo. J Neurosci, 23, 2466-76.

Sah, P. and Faber, E. S. (2002). Channels underlying neuronal calcium-activated potassium currents. Prog Neurobiol, 66, 345-53.

Schiller, P. H., Finlay, B. L. and Volman, S. F. (1976). Short-term response variability of monkey striate neurons. Brain Res, 105, 347-9.

Schwindt, P. C. and Crill, W. E. (1995). Amplification of synaptic current by persistent sodium conductance in apical dendrite of neocortical neurons. J Neurophysiol, 74, 2220-4.

Schwindt, P. C., Spain, W. J. and Crill, W. E. (1988a). Influence of anomalous rectifier activation on afterhyperpolarizations of neurons from cat sensorimotor cortex in vitro. $\mathrm{J}$ Neurophysiol, 59, 468-81.

Schwindt, P. C., Spain, W. J. and Crill, W. E. (1989). Long-lasting reduction of excitability by a sodium-dependent potassium current in cat neocortical neurons. J Neurophysiol, 61, 23344.

Schwindt, P. C., Spain, W. J. and Crill, W. E. (1992). Calcium-dependent potassium currents in neurons from cat sensorimotor cortex. J Neurophysiol, 67, 216-26.

Schwindt, P. C., Spain, W. J., Foehring, R. C., Chubb, M. C. and Crill, W. E. (1988b). Slow conductances in neurons from cat sensorimotor cortex in vitro and their role in slow excitability changes. J Neurophysiol, 59, 450-67. 
Seeburg, P. H. (1993). The TINS/TiPS Lecture. The molecular biology of mammalian glutamate receptor channels. Trends in Pharmacological Sciences, 14, 297-303.

Segev, I. and Rall, W. (1998). Excitable dendrites and spines: earlier theoretical insights elucidate recent direct observations. Trends Neurosci, 21, 453-60.

Shadlen, M. N. and Newsome, W. T. (1994). Noise, neural codes and cortical organization. Curr Opin Neurobiol, 4, 569-79.

Shadlen, M. N. and Newsome, W. T. (1998). The variable discharge of cortical neurons: implications for connectivity, computation, and information coding. J Neurosci, 18, 3870-96.

Sharp, A. A., O'Neil, M. B., Abbott, L. F. and Marder, E. (1993). The dynamic clamp: artificial conductances in biological neurons. Trends Neurosci, 16, 389-94.

Singer, W. (1993). Synchronization of cortical activity and its putative role in information processing and learning. Annu Rev Physiol, 55, 349-74.

Singer, W. and Gray, C. M. (1995). Visual feature integration and the temporal correlation hypothesis. Annu Rev Neurosci, 18, 555-86.

Softky, K. (1993). The highly irregular firing of cortical-cells is inconsistent with temporal integration of random EPSPs. J Neurosci, 13, 334-350.

Softky, W. R. (1995). Simple codes versus efficient codes. Curr Opin Neurobiol, 5, 239-47.

Spain, W. J., Schwindt, P. C. and Crill, W. E. (1991). Two transient potassium currents in layer V pyramidal neurones from cat sensorimotor cortex. J Physiol, 434, 591-607.

Spruston, N., Jaffe, D. B., Williams, S. H. and Johnston, D. (1993). Voltage- and space-clamp errors associated with the measurement of electrotonically remote synaptic events. J Neurophysiol, 70, 781-802. 
Stafstrom, C. E., Schwindt, P. C., Flatman, J. A. and Crill, W. E. (1984). Properties of subthreshold response and action potential recorded in layer $\mathrm{V}$ neurons from cat sensorimotor cortex in vitro. J Neurophysiol, 52, 244-63.

Steriade, M. (2001). Impact of network activities on neuronal properties in corticothalamic systems. J Neurophysiol, 86, 1-39.

Steriade, M., Timofeev, I. and Grenier, F. (2001). Natural waking and sleep states: a view from inside neocortical neurons. J Neurophysiol, 85, 1969-85.

Stevens, C. F. and Zador, A. M. (1998). Input synchrony and the irregular firing of cortical neurons. Nature Neurosci, 1, 210-7.

Stuart, G. (1999). Voltage-activated sodium channels amplify inhibition in neocortical pyramidal cells. Nature Neuroscience, 2, 144-50.

Stuart, G. and Sakmann, B. (1995). Amplification of EPSPs by axosomatic sodium channels in neocortical pyramidal neurons. Neuron, 15, 1065-76.

Stuart, G. and Spruston, N. (1998). Determinants of voltage attenuation in neocortical pyramidal neuron dendrites. J Neurosci, 18, 3501-10.

Tanabe, S., Sato, S. and Pakdaman, K. (1999). Response of an ensemble of noisy neuron models to a single input. Phys Rev E Stat Phys Plasmas Fluids Relat Interdiscip Topics, 60, $7235-8$.

Thomson, A. M. (1997). Activity-dependent properties of synaptic transmission at two classes of connections made by rat neocortical pyramidal axons in vitro. J Physiol, 502, 131-47.

Thomson, A. M., Girdlestone, D. and West, D. C. (1988). Voltage-dependent currents prolong single-axon postsynaptic potentials in layer III pyramidal neurons in rat neocortical slices. J Neurophysiol, 60, 1896-907. 
Tolhurst, D. J., Movshon, J. A. and Dean, A. F. (1983). The statistical reliability of signals in single neurons in cat and monkey visual cortex. Vision Res, 23, 775-85.

Tolhurst, D. J., Movshon, J. A. and Thompson, I. D. (1981). The dependence of response amplitude and variance of cat visual cortical neurones on stimulus contrast. Exp Brain Res, 41, 414-9.

Tsodyks, M. V. and Markram, H. (1997). The neural code between neocortical pyramidal neurons depends on neurotransmitter release probability. Proc Natl Acad Sci U S A, 94, $719-23$.

Villa, A. E. and Abeles, M. (1990). Evidence for spatiotemporal firing patterns within the auditory thalamus of the cat. Brain Res, 509, 325-7.

Volgushev, M., Pei, X., Vidyasagar, T. R. and Creutzfeldt, O. D. (1993). Excitation and inhibition in orientation selectivity of cat visual cortex neurons revealed by whole-cell recordings in vivo. Vis Neurosci, 10, 1151-5.

von der Malsburg, C. (1981) The correlation theory of brain function. Internal Report 81-2. Dept. of Neurobiology, Max-Planck-Institiute for Biophysical Chemistry; Göttingen.

Warzecha, A. K. and Egelhaaf, M. (1999). Variability in spike trains during constant and dynamic stimulation. Science, 283, 1927-30.

White, E. L. (1989) Cortical Circuits. Birkhauser Publishing Inc; Boston.

White, J. A., Klink, R., Alonso, A. and Kay, A. R. (1998). Noise from voltage-gated ion channels may influence neuronal dynamics in the entorhinal cortex. J Neurophysiol, 80 , 262-9.

White, J. A., Rubinstein, J. T. and Kay, A. R. (2000). Channel noise in neurons. Trends Neurosci, 23, 131-7. 
Williams, S. R. and Stuart, G. J. (2000). Site independence of EPSP time course is mediated by dendritic I(h) in neocortical pyramidal neurons. J Neurophysiol, 83, 3177-82.

Yuste, R. and Tank, D. W. (1996). Dendritic integration in mammalian neurons, a century after Cajal. Neuron, 16, 701-16.

Zhu, J. J. and Connors, B. W. (1999). Intrinsic firing patterns and whisker-evoked synaptic responses of neurons in the rat barrel cortex. J Neurophysiol, 81, 1171-83. 


\section{DEFINITIONS AND ABBREVIATIONS}

AHP: Defined as the voltage difference between the threshold and the minimum of afterhyperpolarization after the spike.

Auto renewal function: to measure how the probability of spiking changes after a spike on cells with ongoing activity. In every trace all the spikes were considered as reference points and the timing of spikes following each reference spike in a $100 \mathrm{~ms}$ window were measured and collected in a histogram with a bin size of $1 \mathrm{~ms}$ (see page 30 ).

Background firing frequency was calculated from instantaneous firing rate of those PSTH that were generated without the presence of EPSP, by averaging the bins from $200 \mathrm{~ms}$ from the starting point to the end of the stimulation.

Box car filter: 9 points before and after the smoothed value were averaged together with the smoothed value (see page 32 ).

Cumulative sum: The probability of spiking in time (see page 29).

Cumulative delay in spike timing: calculated from those traces in which an simEPSP contributed to the peak synaptic gain (simEPSP-spike). All the spikes on the control traces that could have been influenced by the simEPSP-spike were characterized by two values: their relative position to the simEPSP-spike, and a "delay", which was calculated from the delay of the corresponding spike in the spike train with simEPSP-spike. If a spike on the control train did not occur due to the simEPSP-spike, then its delay was calculated as a delay to the position of the next spike in the simEPSP-spike train, independent of whether that given spike was related to other spikes in the control spike 
train. In this way the maximum delay was controlled by the frequency. The average delay for spikes that did not occur was the reciprocal value of the frequency (see page 69).

eEPSP: evoked EPSP (see page 18).

Instantaneous firing frequency: firing frequency corresponding to the individual bins of the peristimulus time histogram with a bin size of $1 \mathrm{~ms}$ (page 29).

Loss of spikes: The probability that the spike count of a spike train does not increase after a transient increase due to the EPSP (peak synaptic gain). It is calculated as the average value of the longer, stabilized part of the cumulative sum after the initial peak due to the EPSP, then normalized to the peak synaptic gain (see page 57).

Normalized EPSP amplitude: the ratio of EPSP amplitude measured at membrane potential values near spike threshold and at resting potentials (see page 39).

Normalized EPSP half width: the ratio of EPSP half widths measured at membrane potentials near spike threshold and at resting potentials. (see page 37).

Normalized EPSP rise time: which was defined as the ratio (depolarized/resting) of the EPSP rise time obtained at membrane potentials near spike threshold and at resting potentials (see page 41).

Peak synaptic gain: probability of spiking due to the EPSP. It was defined as the maximum on the cumulative sum histogram after the onset of the EPSP (see page 30).

Peristimulus time histogram: a histogram of spike timing collected from a large number of traces, locked to the timing of the stimulus (see page 29).

Perturbation window: defines a period following the simEPSP-related spike, after which the probability that position of a spike generated by the background activity, is changed because of the presence of the simEPSP-spike, is less than 5\%. The perturbation window 
was calculated as the time where the cumulative delay in spike timing reached $95 \%$ of the plateau value (see page 70).

Probability of firing: The chance that an EPSP evoke a spike when the cell is depolarized to near spike threshold (see page 37).

PSTH: peristimulus time histogram

SimEPSP: simulated EPSP that were generated by injection of a double exponential shaped current transient (see page 22.)

Spike amplitude $(\mathrm{mV})$ : measured between the spike threshold and maximum of the spike

Spike half width (ms): measured between the points defined the spike amplitude at 50\% size between the rise phase and decay phase of the spike.

Spike time window: Defined to quantify the distribution of spike timing. Cumulative probabilities of firing in time are calculated from the spike latencies from the onset of the EPSP. Spike time window was measured between the points corresponding to 0.1 and 0.9 values of histogram of the cumulative probabilities firing (see page 50).

EPSP half width (ms): time between the rising and decay phase of EPSP at half the amplitude (see page 18).

EPSP rise time (ms): time during the rising phase of the EPSP between the $10 \%$ and $90 \%$ levels of amplitude (see page 18).

Width of the peak synaptic gain (ms): defined as a window between the 10 and $90 \%$ of the maximum on the cumulative sum. 


\section{VITA}

Veronika Zsiros was born in Kazincbarcika, Hungary. She got her M.Sc. degree in Molecular biology and Biotechnology at the University of Szeged, Hungary (formerly József Attila University of Arts and Sciences) in 1995. She also received a B.Sc degree in Computer Science at the University of Szeged in 1997. 